\title{
WestVirginiaUniversity
}

THE RESEARCH REPOSITORY @ WVU

Graduate Theses, Dissertations, and Problem Reports

2004

\section{FEM simulation of ultrasonic wave propagation in solid rods}

\author{
Tuan-Chun Fu \\ West Virginia University
}

Follow this and additional works at: https://researchrepository.wvu.edu/etd

\section{Recommended Citation}

Fu, Tuan-Chun, "FEM simulation of ultrasonic wave propagation in solid rods" (2004). Graduate Theses, Dissertations, and Problem Reports. 1429.

https://researchrepository.wvu.edu/etd/1429

This Thesis is protected by copyright and/or related rights. It has been brought to you by the The Research Repository @ WVU with permission from the rights-holder(s). You are free to use this Thesis in any way that is permitted by the copyright and related rights legislation that applies to your use. For other uses you must obtain permission from the rights-holder(s) directly, unless additional rights are indicated by a Creative Commons license in the record and/ or on the work itself. This Thesis has been accepted for inclusion in WVU Graduate Theses, Dissertations, and Problem Reports collection by an authorized administrator of The Research Repository @ WVU. For more information, please contact researchrepository@mail.wvu.edu. 


\title{
FEM Simulation of Ultrasonic Wave Propagation in Solid Rods
}

\author{
Tuan-Chun $\mathrm{Fu}$
}

Thesis submitted to the

College of Engineering and Mineral Resources

at West Virginia University

in partial fulfillment of the requirements

for the degree of

Master of Science
in
Civil Engineering

Roger H. L. Chen, Ph.D., Chair

Hota V. GangaRao, Ph.D.

H. Ilkin Bilgesu, Ph.D.

West Virginia University

Morgantown, West Virginia

2004

Keywords: ultrasonic, wave, propagation, solid, rod, prestress 


\section{ABSTRACT \\ FEM Simulation of Ultrasonic Wave Propagation in Solid Rods}

\section{TUAN-CHUN FU}

The main objective of this study is to simulate ultrasonic wave propagation in solid cylindrical rods. In this study, both experimental investigations and theoretical analyses are conducted. In the experiment, the $4.2 \mathrm{~mm}$ diameter center wire of the commonly used Grad 2507 -wire strand have been employed as the specimen and all the specimens are under zero tensile force. There are 3 rods (center wire) of different lengths used in this study. The DS345 arbitrary function generator, PICO sensors, and S9208 displacement transducer is used for measuring the results of the ultrasonic wave propagations. In the FEM analyses, each specimen with the same input is generated for comparing with the experimental results. In addition, the input frequency, element size, and integration time step are discussed to evaluate the accuracy of the FEM results. The output waveform, wave velocity, and frequency components are compared between experimental and FEM results. A time-frequency domain tool, Wigner-Ville Transform (WVT), is also employed to provide better information regarding frequency dispersion and the concentration of energy under a certain frequency input. The comparisons show that results correlate well, and good measurement accuracy is observed, indicating that FEM can also be an efficient method to simulate the ultrasonic wave propagation in an elastic cylindrical rod. This technique can be used to develop the data base for certain pre-stressing tendons used in concrete structures in order to determine the residual force or even defects in the strand in the future. 


\section{ACKNOWLEDGMENTS}

The author would like to express tremendous thanks to his advisor, Dr. Roger H. L. Chen, for his guidance and advice throughout the research and preparation of this thesis. Appreciation is also extended to Dr. Hota V. GangaRao and Dr. H. Ilkin Bilgesu, members of the advisory committee, for their constructive comments and suggestions.

The author wishes to acknowledge Dr. Komwut Wissawapaisal and Dr. Alejandro C. Kiriakidis L. for their technical assistance. Appreciation is also extended to Mr. Joseph Sweet and Mr. Joshua Cook in the preparation of this thesis.

Finally, the author wishes to express his deep gratitude to his parents, Mrs. Nancy Wu and Dr. Jene-John Fu for their unconditional love, encouragement and understanding. He would like to convey a special word of thanks to Hsi-Wen Chen and Ming-Yi Fu for their constant help and seeing him through this ordeal. 


\section{TABLE OF CONTENTS}

ABSTRACT .ii

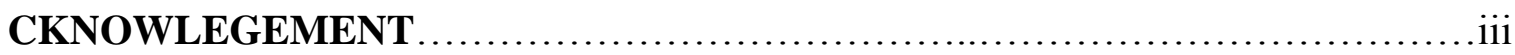

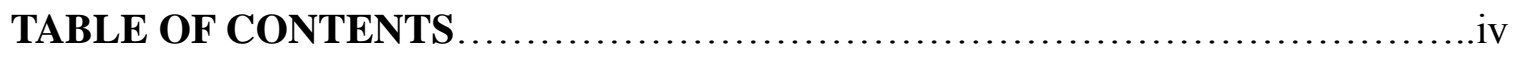

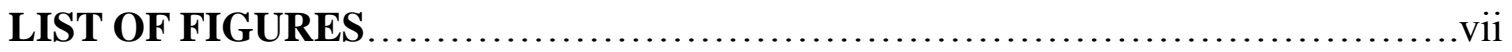

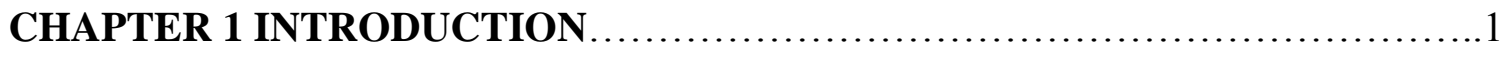

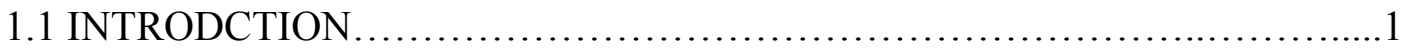

1.2 MEASUREMENT OF WAVEFORM IN SOLID ROD.......................2

1.3 RESEARCH OBJECTIVES AND SCOPES...................................3

1.3.1 Objectives

1.3.2 Scopes

1.4 ORGANIZATIN OF THE THESIS

CHAPTER 2 LITERATURE REVIEW ................................................

2.1 ULTRASONIC TESTING.............................................. 5

2.2 WAVE PROPAGATION...................................................

2.3 WIGNER-VILLE TRANSFORM...................................... 6

2.4 FINITE ELEMENT METHOD.......................................

CHAPTER 3 EXPERIMENTAL RESULT OF WAVE PROPAGATION IN

SOLID RODS UNDER FREE TENSILE FORCE...................12

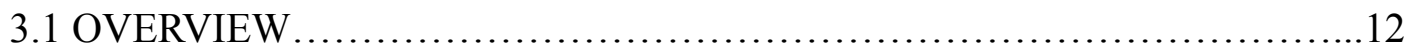

3.2 INSTRUMENTATION................................................ 12 
3.3 PROCEEDING OF THE EXPERIMENT ....................................15

3.3.1 Short Rod..................................................... 15

3.3.2 Long Rod......................................................... 16

3.3.3 Experiment Process............................................... 16

3.4 SHORT ROD EXPERIMENT RESULTS...............................17

3.5 LONG ROD EXPERIMENTAL RESULTS...............................17

3.6 EXPERIMENTAL RESULTS OBSERVATION AND DICUSSION...........18

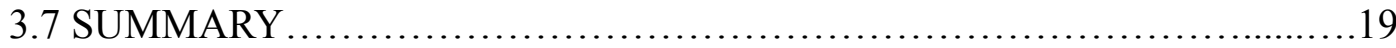

\section{CHAPTER 4 THE APPLICATION OF FINITE ELEMENT METHOD FOR}

ULTRASONIC WAVE PROPAGATION IN A SOLID ROD............30

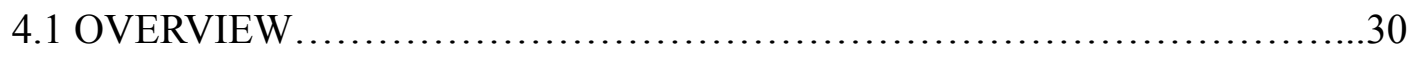

4.2 INTRODUCTION OF FEM SOFTWARE.................................. 30

4.2.1 3-D (Brick) Model...................................................

4.2.2 Axis-Symmetric Model............................................ 32

4.3 COMPARSION BETWEEN 3-D AND AXIS-SYMMETRIC MODEL..........32

4.4 THE DETERMINATION OF LOADING FUNCTION, LOADING FREQUENCY, ELEMENT SIZE AND TIME STEP.........................34

4.4.1 The Frequency Components of the Input Loading Functions................34

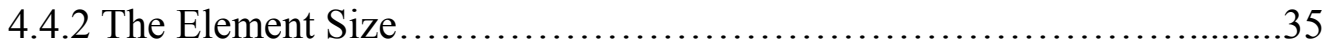

4.4.3 The Integration Time Step..........................................

4.5 COMPARING ALGOR FEM OUTPUT WITH BOOK RESULT..............37

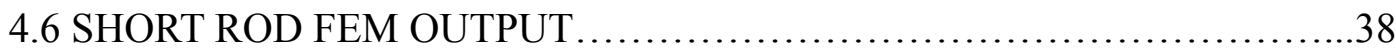

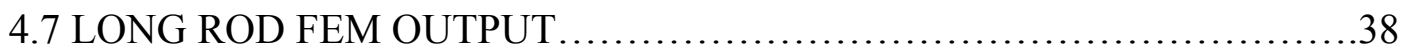


CHAPTER 5 COMPARISON BETWEEN EXPERIMENTAL RESULT AND FINITE ELEMENT METHODS OUTPUT ..............................50

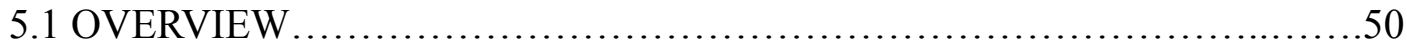

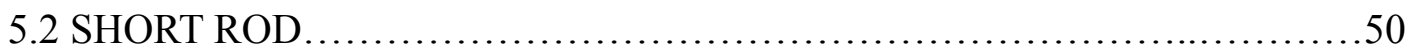

5.2.1 Arrival Time..........................................................

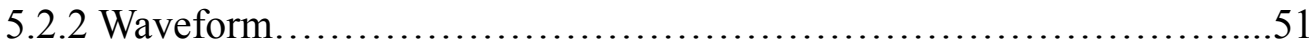

5.2.3 Wigner-Ville Transform (WVT) .................................. 51

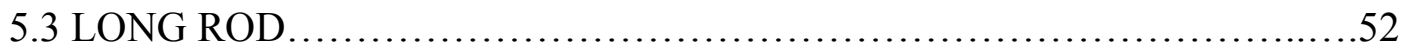

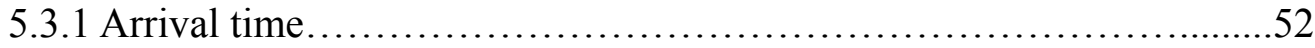

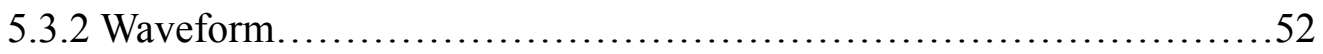

5.3.3 Wigner-Ville Transform........................................53

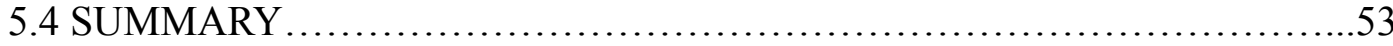

CHAPTER 6 CONCLUSIONS AND RECOMMENDATIONS....................76

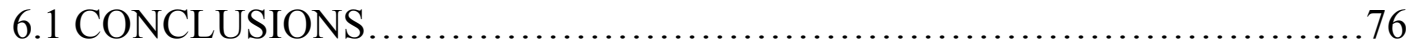

6.2 RECOMMENDATION FOR FUTURE STUDY ...........................78

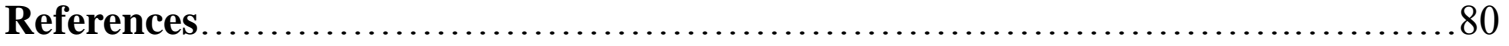

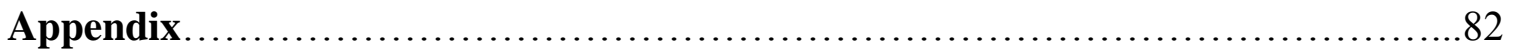




\section{LIST OF FIGURES}

Figure 3.1 The typical waveform at the receiving end of the rod................... 21

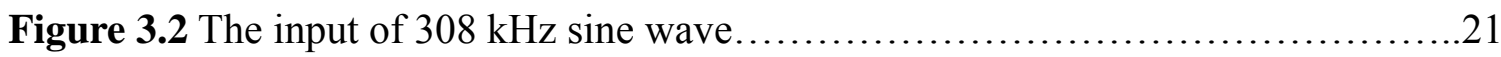

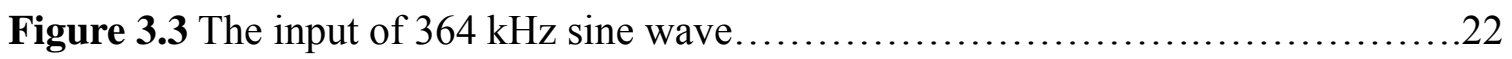

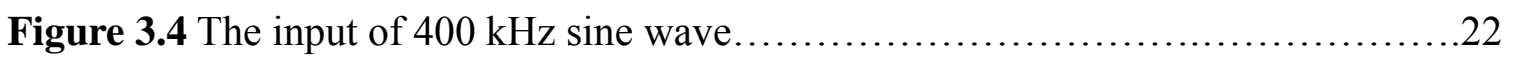

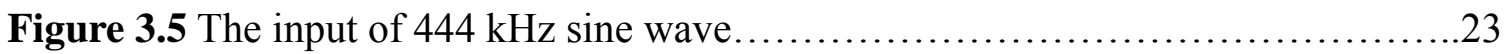

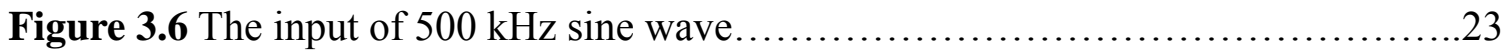

Figure 3.7 The input of the pulse generator...................................... 24

Figure 3.8 The $308 \mathrm{kHz}$ sine wave output of the short rod........................24

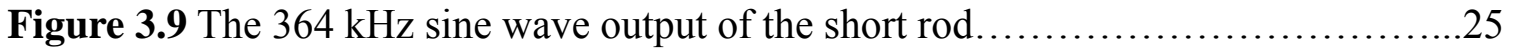

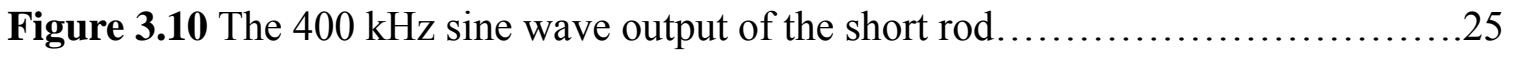

Figure 3.11 The $444 \mathrm{kHz}$ sine wave output of the short rod........................26

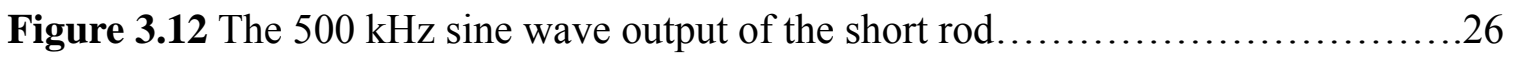

Figure 3.13 The output generated by $\mathrm{C} 101 \mathrm{HV}$ on the short rod.....................27

Figure 3.14 The output generated by $\mathrm{C} 101 \mathrm{HV}$ pulse generator on $4.76 \mathrm{~m}$ long rod (Chen and Wissawapaisal, 2001)................................27

Figure 3.15 The $500 \mathrm{kHz}$ sine wave output of the 3 meter single strand...............28

Figure 3.16 The zoom in of the 5.15 (present the wave arrival time with more clarity)...28

Figure 3.17 The output wave signal generated by pulse generator recorded on the receiving end of the 3 meter rod.

Figure 4.1 The comparison of the cross section between 3-D model and Axis-

Symmetricmodel. Gray area shows the error between these two types of model........................................................... 40

Figure 4.2 The schematic drawing of the FEM simulation.

Figure 4.3 Group velocity of the first two longitudinal modes for the center wire $(\varnothing=4.2 \mathrm{~mm})$. There is no tensile force applied

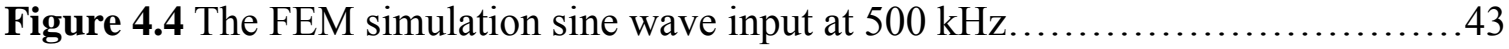

Figure 4.5 The Half Hanning function input..................................43

Figure 4.6 Comparison of Miklowitz's experiment result, 3D FEM model result, and Axis-Sym FEM model result.....................................44

Figure 4.7 The FEM output of the $308 \mathrm{kHz}$ sine wave on the short rod.................44

Figure 4.8 The FEM output of the $364 \mathrm{kHz}$ sine wave on the short rod.................45

Figure 4.9 The FEM output of the $400 \mathrm{kHz}$ sine wave on the short rod................45

Figure 4.10 The FEM output of the $444 \mathrm{kHz}$ sine wave on the short rod...............46 
Figure 4.11 The FEM output of the $500 \mathrm{kHz}$ sine wave on the short rod..................46

Figure 4.12 The FEM output of the $308 \mathrm{kHz}$ sine wave on the 3 meters rod................47

Figure 4.13 The FEM output of the $364 \mathrm{kHz}$ sine wave on the 3 meters rod...............47

Figure 4.14 The FEM output of the $400 \mathrm{kHz}$ sine wave on the 3 meters rod...............48

Figure 4.15 The FEM output of the $444 \mathrm{kHz}$ sine wave on the 3 meters rod................48

Figure 4.16 The FEM output of the $500 \mathrm{kHz}$ sine wave on the 3 meters rod................49

Figure 4.17 The FEM output of the pulse generator input on the 3 meters rod.............49

Figure 5.1 The waveform comparison between the experimental and FEM result from 308

$\mathrm{kHz}$ sine wave input.................................................. 55

Figure 5.2 The waveform comparison between the experimental and FEM result from

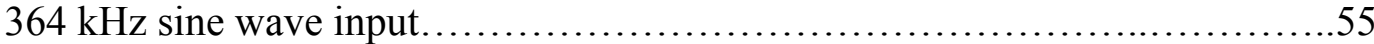

Figure 5.3 The waveform comparison between the experimental and FEM result from $400 \mathrm{kHz}$ sine wave input...........................................56

Figure 5.4 The waveform comparison between the experimental and FEM result from

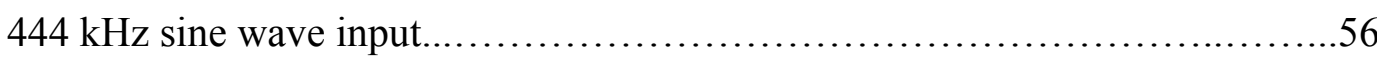

Figure 5.5 The waveform comparison between the experimental and FEM result from

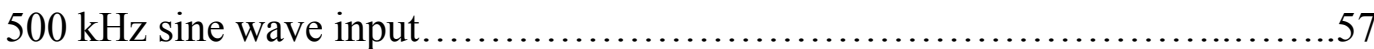

Figure 5.6 The waveform comparison between the experimental and FEM result from the C101HV pulse generator input......................................57

Figure 5.7 The Wigner-Ville Transform of $308 \mathrm{kHz}$ sine wave experiment output on the

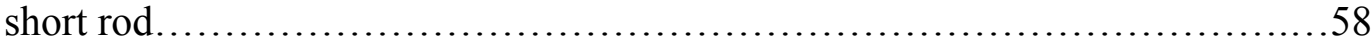

Figure 5.8 The Wigner-Ville Transform of $308 \mathrm{kHz}$ sine wave FEM output on the short

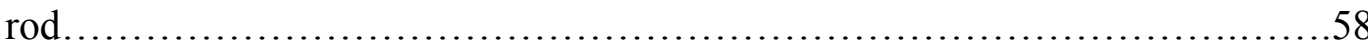

Figure 5.9 The Wigner-Ville Transform of $364 \mathrm{kHz}$ sine wave experiment output on the short rod.

Figure 5.10 The Wigner-Ville Transform of $364 \mathrm{kHz}$ sine wave FEM output on the short

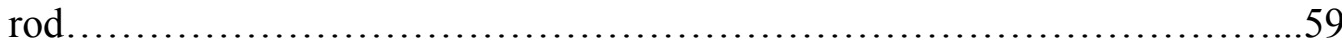

Figure 5.11 The Wigner-Ville Transform of $400 \mathrm{kHz}$ sine wave experiment output on the

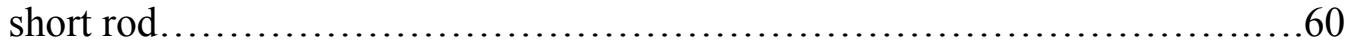

Figure 5.12 The Wigner-Ville Transform of $400 \mathrm{kHz}$ sine wave FEM output on the short rod.

Figure 5.13 The Wigner-Ville Transform of $444 \mathrm{kHz}$ sine wave experiment output on the short rod....

Figure 5.14 The Wigner-Ville Transform of $444 \mathrm{kHz}$ sine wave FEM output on the short $\operatorname{rod}$ 
Figure 5.15 The Wigner-Ville Transform of $500 \mathrm{kHz}$ sine wave experiment output on the short rod.

Figure 5.16 The Wigner-Ville Transform of $500 \mathrm{kHz}$ sine wave FEM output on the short $\operatorname{rod}$

Figure 5.17 The Wigner-Ville Transform of pulse experiment output on the short rod.......63

Figure 5.18 The Wigner-Ville Transform of pulse FEM output on the short rod............63

Figure 5.19 The waveform comparison between the experimental and FEM result from 308

$\mathrm{kHz}$ sine wave input on the 3 meters rod.

Figure 5.20 The waveform comparison between the experimental and FEM result from 364

$\mathrm{kHz}$ sine wave input on the 3 meters rod.

Figure 5.21 The waveform comparison between the experimental and FEM result from 400

$\mathrm{kHz}$ sine wave input on the 3 meters rod.

Figure 5.22 The waveform comparison between the experimental and FEM result from 444

$\mathrm{kHz}$ sine wave input on the 3 meters rod.

Figure 5.23 The waveform comparison between the experimental and FEM result from 500

$\mathrm{kHz}$ sine wave input on the 3 meters rod.

Figure 5.24 The zoom-in experimental waveform from $308 \mathrm{kHz}$ sine wave input on the 3 meters rod.

Figure 5.25 The zoom-in experimental waveform from $364 \mathrm{kHz}$ sine wave input on the 3 meters rod.

Figure 5.26 The zoom-in experimental waveform from $400 \mathrm{kHz}$ sine wave input in the 3 meters rod.

Figure 5.27 The zoom-in experimental waveform from $444 \mathrm{kHz}$ sine wave input on the 3 meters rod.

Figure 5.28 The zoom-in experimental waveform from $500 \mathrm{kHz}$ sine wave input on the 3 meters rod.

Figure 5.29 The waveform comparison between the experimental and FEM result from the C101HV pulse generator input on the 3 meters rod.

Figure 5.30 The Wigner-Ville Transform applied on experimental result due to the $308 \mathrm{kHz}$

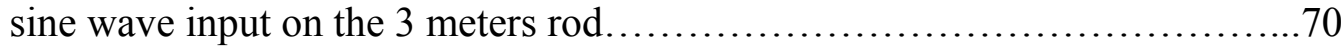

Figure 5.31 The Wigner-Ville Transform applied on FEM result due to the $308 \mathrm{kHz}$ sine wave input on the 3 meters rod...................................... 70

Figure 5.32 The Wigner-Ville Transform applied on experimental result due to the $364 \mathrm{kHz}$

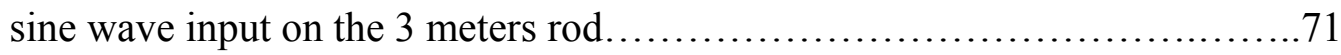

Figure 5.33 The Wigner-Ville Transform applied on FEM result due to the $364 \mathrm{kHz}$ sine wave input on the 3 meters rod. 
Figure 5.34 The Wigner-Ville Transform applied on experimental result due to the $400 \mathrm{kHz}$ sine wave input on the 3 meters rod..................................... 72

Figure 5.35 The Wigner-Ville Transform applied on FEM result due to the $400 \mathrm{kHz}$ sine wave input on the 3 meters rod........................................ 72

Figure 5.36 The Wigner-Ville Transform applied on experimental result due to the $444 \mathrm{kHz}$ sine wave input on the 3 meters rod..................................73

Figure 5.37 The Wigner-Ville Transform applied on FEM result due to the $444 \mathrm{kHz}$ sine wave input on the 3 meters rod....................................... 73

Figure 5.38 The Wigner-Ville Transform applied on experimental result due to the $500 \mathrm{kHz}$ sine wave input on the 3 meters rod.................................... 74

Figure 5.39 The Wigner-Ville Transform applied on FEM result due to the $500 \mathrm{kHz}$ sine wave input on the 3 meters rod..................................... 74

Figure 5.40 The Wigner-Ville Transform applied on experimental result due to the C101HV pulse generator input on the 3 meters rod................................ 75

Figure 5.41 The Wigner-Ville Transform applied on FEM result due to the C101HV pulse generator input on the 3 meters rod.................................. 75 


\section{CHAPTER 1 \\ INTRODUCTION}

\subsection{INTRODUCTION}

There is an increasing need for health monitoring of civil engineering structures. Different kinds of nondestructive evaluation (NDE) have already been developed, and each of the NDE method has its own advantages and limitations. The most commonly used NDE methods are radiography, eddy current, magnetic flux leakage, and acoustic monitoring.

The method presented in this thesis uses ultrasonic mechanical waves which are propagated through the whole length of the seven-wire strands. A stress wave is instigated at one end of the strand and detected at the other end. According to the experimental results, the wave form dispersion can be observed due to the fact that lower frequencies travel faster than higher frequencies within metal. One limitation of the experimental results is the attenuation caused by boundary conditions. Attenuation is the absorption of energy as a result of the waves after traveling a long distance or coming into contact with concrete (boundary condition). Consequently the wave energy will disappear and cannot reach the other end of the stress strand. Computer simulations become essential to the purpose of our experiment, because they eliminate the limitations posed by the boundary conditions.

One of the most effective computer simulation methods is the finite element method (FEM). Using FEM, we can generate the model which is to proximate the steel in the real life and simulate the same stress wave input to observe the output wave form on the other end of the strand. 
The purpose of this study is to generate an effective model to make the FEM simulation successful. This depends on the element size, the frequency of input waveform and the material properties.

\subsection{MEASUREMENT OF WAVEFROM IN SOLID ROD}

In order to measure the waveform in a solid rod, a stress wave is sent through it by utilizing a pulse generator. Using a sensor the waveform can be detected at the opposite end of the rod. Due to technological developments we can have precise function generators and sensors to produce and detect the waveforms. In this thesis, we will compare the experimental results and FEM output via waveform, wave velocity, frequency content, and attenuation.

The measurement parameters will typically be one or more of the following:

1. Wave velocity/wavelength traveling time: Wave velocity is the easiest ultrasonic parameter to measure. The wave velocity changes because of material properties, frequency content, etc.

2. Frequency content (Spectrum): When an ultrasonic wave travels in a solid rod, it will separate by different frequencies, which is most notable in longer rods. Having that low frequency waves travel faster than higher frequency waves we need to apply a Time-Frequency domain transform such as Winger-Ville Transform.

3. Attenuation: When a wave is traveling in any material, the energy of a wave will be absorbed or attenuated by the material. The attenuated rate is due to the material properties and boundary conditions of the test specimen. When we compare the output waveform of actual experimental results with FEM results, it is found that the 
attenuation of FEM output waveform will be much lower than the experimental one.

\subsection{RESEARCH OBJECTIVES AND SCOPES}

\subsubsection{Objectives}

The main objectives of this study are to:

1. Develop a computer simulation model for wave propagation in a solid rod.

2. Investigate the behavior of a stress wave dispersing in a strand by experimental and FEM methods, and compare the results.

3. Apply the signal analysis technique (Wigner-Ville Transform) on the output stress waveform to analyze the frequency content in the time domain.

\subsubsection{Scopes}

The scopes of this study include the analysis and experimental testing of wave propagation in a 3/16 inch diameter, Grad 250 seven-wire strand, which is generally used in pre-stressing construction. An ultrasonic stress wave is generated to measure both the wave dispersion and the wave velocity. The analysis of the ultrasonic waveform using Wigner-Ville Transform is used to observe how the wave disperses. Algor program, a FEM program, is used to simulate the ultrasonic wave propagating in a solid rod. The theoretical study is based on the frequency equation of a waveguide with a circular cross section. The material property effects are also taken into consideration. 


\subsection{ORGANIZATION OF THE THESIS}

There are 6 chapters in this thesis. Chapter 1 presents the introduction of this study. Chapter 2 presents the literature review which talks about some earlier studies and experiments which are related to this study. Chapter 3 describes the experimental setup for testing the ultrasonic wave propagation in a solid rod. Chapter 4 presents the FEM. Chapter 5 will compare the FEM output with experimental results. This will demonstrate the use of an efficient signal processing technique in order to establish the relationships between the frequency and the traveling time. Chapter 6 will include conclusions and recommendations. 


\section{CHAPTER 2}

\section{LITERATURE REVIEW}

\subsection{ULTRASONIC TESTING}

Research on ultrasonic wave propagation techniques was first accomplished by Langevin of France during World War II. However, these techniques were mainly applied for military proposes. Lengevin was able to detect a submarine at a distance of $1.5 \mathrm{Km}$. In 1927, Boyle, Wood and Loomis used quartz piezoelectric to generate ultrasonic waves. During 1927 and 1935, Sokolov mentioned using ultrasonic waves to detect the defects in metal objects. He has done a lot of pioneering research in ultrasonic testing such as developing the method of obtaining an image of a structural defect. In 1940, Schraiber accomplished the method of continuous ultrasonic wave testing.

Currently, most of the ultrasonic testing methods are based on pulsed ultrasonic waves, which was developed by Firstone (1940) and Simmons (1945). This method is relatively simple and effecient. From these early beginnings, the ultrasonic testing method has depended on advanced technology. Due to the development of sensor, function generator, and signal processing skill, ultrasonic testing have become wildly used in many fields.

\subsection{WAVE PROPAGATION}

Hsieh and Kolsky (1957) compared the ultrasonic wave output between theoretical calculations and experimental observations of the displacement of a $22.5 \mathrm{~cm}$ in length and 
$0.63 \mathrm{~cm}$ in diameter aluminum rod. Their conclusions showed that the displacement of the cross section calculated appears to be close to the experimental result. In 1958, Folk, Fox and Curtis reported a research which showed when the loading rate of stress wave is rapid, the strain pulse would spread out during traveling time and develop oscillations. Fox and Curtis carried out an experimental observation to compare the theoretical solutions. In 1961, Meitzler mentioned about the mode coupling occurring in the propagation of elastic pulses in wires. Chen and Wissawapaisal (2002) reported a relationship between the stress and the ultrasonic wave in solid rod by employing an efficient signal proceeding technique, Wigner-Ville Transform, which can transform the ultrasonic waveform into time-frequency domain. This thesis will focus on how to use Finite Element Method to simulate the wave propagation in solid rod, and applying the Wigner-Ville Transform on the FEM results.

\subsection{WIGNER-VILLE TRANSFORM}

In most situations, time domain on the $\mathrm{x}$-axis is most commonly used with ultrasonic wave diagrams. However, transforming those signals into the frequency-amplitude domain is another important and necessary component to describe signals. While frequency domain can nicely show the strength of various spectral components, the temporal locations of these components cannot be seen in a frequency domain type spectrum. The desire of representing temporal and spectral element of certain signals would require the use of the time-frequency representation.

The Wigner-Ville Transform (WVT) transforms the real-time wave signal to time-frequency domain. This gives us better explanation of the frequency delay and propagation under different velocities. In addition, WVT is one of the modern 
high-resolution spectrum simulation methods which provide more possibilities of analyzing highly distorted signals. WVT is also useful for our non-stationary structure response. The Winger-Ville Transform, W(t,f), of a signal $s(t)$ is defined as Eq. (2.1)

$$
w(t, f)=\int_{\tau} s(t+\tau / 2) \times s^{*}(t-\tau / 2) \times e^{-i 2 \pi f \tau} d \tau
$$

where $\mathrm{s}^{*}(\mathrm{t})$ is the complex conjugate of the $\mathrm{s}(\mathrm{t})$. The $2 \pi \mathrm{f}$ is equal to $\omega$ where frequency is the variable.

The WVT is a two-dimensional function which describes the frequency content as a function of the time. Hence, WVT also possesses many advantageous properties. For example:

1. With the time duration of $t$, the instantaneous frequency of the $w(t, f)$ is equal to the mean frequency of the signal.

2. The energy distribution in $\mathrm{w}(\mathrm{t}, \mathrm{f})$ is equal to the energy in the original signal. This advantage helps us to locate the peak energy of a certain frequency band of the signal.

\subsection{The Finite Element Method}

The direct integration method is efficient for many structural dynamic and wave propagation problems. In step-by-step methods, the following equation is used as the control equation. 


$$
[M]\{\ddot{D}\}_{n}+[C]\{\dot{D}\}_{n}+[K]\{D\}_{n}=\left\{R^{e x t}\right\}_{n}
$$

where the $\{D\}$ means the displacement, and the $\{\ddot{D}\}$ and $\{\dot{D}\}$ mean the second and the first order difference of the displacement $\{D\}$. The subscript $\mathrm{n}$ donates time $[\mathrm{n} \Delta \mathrm{t}]$ and the $\Delta t$ is the size of time increment or time step. For the nonlinear problems, $[K]$ is a function of displacement and therefore of time as well. This means we may change the term $[K]\{D\}_{n}$ to $\left\{R^{\text {int }}\right\}_{n}$. The $[M]$ and $[C]$ matrixes are set as time-independent, although for some problems these maybe nonlinear also. There are two sorts of methods for solving Eq 2.2. These methods can be sorted as explicit and implicit methods. Explicit methods have the form

$$
\{D\}_{n+1}=f\left(\{D\}_{n},\{\dot{D}\}_{n},\{\ddot{D}\}_{n},\{D\}_{n-1} \ldots\right)
$$

and implicit methods have the form

$$
\{D\}_{n+1}=f\left(\{\dot{D}\}_{n+1},\{\ddot{D}\}_{n+1},\{D\}_{n}, \ldots\right)
$$

Hence permit $\{D\}_{n+1}$ require the time derivatives of the $\{D\}_{n+1}$, which is unknown in implicit methods. Having Equation 2.3 and 2.4, it is obvious that these two methods have markedly different properties.

In the explicit direct integration methods, it approximates velocity and acceleration by

$$
\{\dot{D}\}_{n}=\frac{1}{2 \Delta t}\left(\{D\}_{n+1}-\{D\}_{n-1}\right)
$$




$$
\{\ddot{D}\}_{n}=\frac{1}{\Delta t^{2}}\left(\{D\}_{n+1}-2\{D\}_{n}+\{D\}_{n-1}\right)
$$

Eq. 2.5 and 2.6 are obtained by expanding terms $\{D\}_{n+1}$ and $\{D\}_{n-1}$ in Taylor Series about time $\mathrm{n} \Delta \mathrm{t}$ :

$$
\begin{aligned}
& \{D\}_{n+1}=\{D\}_{n}+\Delta t\{\dot{D}\}_{n}+\frac{\Delta t^{2}}{2}\{\ddot{D}\}_{n}+\frac{\Delta t^{3}}{6}\{\dddot{D}\}_{n}+\cdots \\
& \{D\}_{n-1}=\{D\}_{n}-\Delta t\{\dot{D}\}_{n}+\frac{\Delta t^{2}}{2}\{\ddot{D}\}_{n}-\frac{\Delta t^{3}}{6}\{\dddot{D}\}_{n}+\cdots
\end{aligned}
$$

subtracting Eq 2.8 from Eq. 2.7 yield Eq. 2.5 while adding Eq. 2.7 and 2.8 yields Eq. 2.6. The terms higher than $\Delta \mathrm{t}^{2}$ are omitted. Combining Eq 2.5 and Eq. 2.6 with Eq. 2.2 yields

$$
\left[\frac{1}{\Delta t^{2}} M+\frac{1}{2 \Delta t} C\right]\{D\}_{n+1}=\left\{R^{e x t}\right\}_{n}-[K]\{D\}_{n}+\frac{1}{\Delta t^{2}}[M]\left(2\{D\}_{n}-\{D\}_{n-1}\right)+\frac{1}{2 \Delta t}[C]\{D\}_{n-1}
$$

The common implicit direct integration methods are unconditionally stable and have no restriction on the time step size for accuracy. A popular unconditionally stable method is called the trapezoidal rule (average acceleration method). This rule approximate the velocity and acceleration by

$$
\begin{aligned}
& \{D\}_{n+1}=\{D\}_{n}+\frac{\Delta t}{2}\left(\{\dot{D}\}_{n}+\{\dot{D}\}_{n+1}\right) \\
& \{\dot{D}\}_{n+1}=\{\dot{D}\}_{n}+\frac{\Delta t}{2}\left(\{\ddot{D}\}_{n}+\{\ddot{D}\}_{n+1}\right)
\end{aligned}
$$


Also, Eq. 2.10 and Eq. 2.11 can be obtained by using Taylor series to expand $\{\dot{D}\}_{n+1}$ and $\{\ddot{D}\}_{n+1}$. Solving $\{\dot{D}\}_{n+1}$ and $\{\ddot{D}\}_{n+1}$ with Eq. 2.10 an Eq. 2.11 can provide

$$
\begin{aligned}
& \{\dot{D}\}_{n+1}=\frac{2}{\Delta t}\left(\{D\}_{n+1}-\{D\}_{n}\right)-\{\dot{D}\}_{n} \\
& \{\ddot{D}\}_{n+1}=\frac{4}{\Delta t^{2}}\left(\{D\}_{n+1}-\{D\}_{n}\right)-\frac{4}{\Delta t}\{\dot{D}\}_{n}-\{\ddot{D}\}_{n}
\end{aligned}
$$

Combine Eq. 2.2, Eq. 2.12, and Eq. 2.13 at time $(\mathrm{n}+1) \Delta \mathrm{t}$, yields

$$
\left[K^{\text {eff }}\right]\{D\}_{n+1}=\left\{R^{\text {eff }}\right\}_{n+1}
$$

where effective stiffness matrix and effective load matrix are

$$
\begin{gathered}
{\left[K^{e f f}\right]=\frac{4}{\Delta t^{2}}+\frac{2}{\Delta t}[C]+[K]} \\
\left\{R^{e f f}\right\}_{n+1}=\left\{R^{e x t}\right\}_{n+1}+[M]\left(\frac{4}{\Delta t^{2}}\{D\}_{n}+\frac{4}{\Delta t}\{\dot{D}\}_{n}+\{\ddot{D}\}_{n}\right)+[C]\left(\frac{2}{\Delta t}\{D\}_{n}+\{\dot{D}\}_{n}\right)
\end{gathered}
$$

The choice of method of time-history analysis is strongly problem-dependent. In wave propagation problems, the excitation is usually high-frequency components. It is different from the structure dynamic problems because the excitation and response in structure dynamic problems are dominated by low-frequency components. In addition, the element size and the time step in wave propagation problems are markedly small. In this study, the element size is about $0.15 \mathrm{~mm}$ and the time step is about $0.01 \mu \mathrm{sec}$. With the 
explicit methods of direct integration, stability requires small time step for accuracy result. Here the stability restriction is not a serious disadvantage because very small time step $(0.01 \mu \mathrm{sec})$ has been used in this research.

The advantage of the implicit methods over explicit methods is that the implicit methods allow big time setup since they are unconditionally stable. Since we use small time step for accuracy in wave propagation problems, it would be expensive to use implicit methods. For long duration structural dynamic problems, the implicit methods would be more efficient than explicit methods.

Algor FEM software uses implicit methods to solve the wave propagation equations. The 1-D and 3-D wave propagation equations are shown.

1-D wave motion equation

$$
E A \frac{\partial^{2} \varphi(x, t)}{\partial x^{2}}+\rho A \frac{\partial^{2} \varphi(x, t)}{\partial t^{2}}=0
$$

3-D wave equation

$$
E A\left(\frac{\partial^{2} \varphi(x, y, z, t)}{\partial x^{2}}+\frac{\partial^{2} \varphi(x, y, z, t)}{\partial y^{2}}+\frac{\partial^{2} \varphi(x, y, z, t)}{\partial z^{2}}\right)+\rho A \frac{\partial^{2} \varphi(x, y, z, t)}{\partial t^{2}}=0
$$




\section{CHAPTER 3}

\section{EXPERIMENTAL RESULT OF WAVE PROPAGATION IN SOLID RODS UNDER FREE TENSILE FORCE}

\subsection{OVERVIEW}

In this chapter, we will describe the experimental method to understand the actual behavior of stress wave propagation in solid rod. The experimental part of this study will focus on getting the arrival time, and frequency component of the waveform. There are three different lengths of specimens in this experiment. They are:

1. $158.75 \mathrm{~mm}$ short rod,

2. $4.76 \mathrm{~m}$ long rod (Chen and Wissawapaisal, 2002), and

$3.3 \mathrm{~m}$ long rod

All of these three are Grad 250 rods. The short rod and 3 meter rods are input to the center wire of the seven-wire strand; and the 4.76 meter long rod is a seven-wire stress-relived strand. Theoretical studies and analyses are described in accordance with experimental results. For example, figure 3.1 shows the typical wave form detected by the S9208 displacement transducer.

\subsection{INSTRUMENTATION}

\section{$\underline{\text { Physical Acoustics Corporation C101HV Pulse Generator }}$}

The C101HV pulse generator was used in this research. The type of connectors 
between the C101HV pulse generator and the sensors are both $50 \mathrm{Ohms} \mathrm{BNC}$ connectors. This pulse generator can generate up to 400 volts output pulses. In this study, we used the volt level of 30 Volts. The peak frequency of the pulses is around $750 \mathrm{kHz}$. Each generated pulse is manually controlled by hitting a pulse button on the back panel of the C101HV.

\section{DS345 Function and Arbitrary Waveform Generator by Stanford Research System}

The DS345 is a full-featured $30 \mathrm{MHz}$ synthesized function generator. It generates several types of standard waveform such as sine wave, square wave, or triangular wave with high frequency resolutions. The DS345 generator can generate sine waves up to $30 \mathrm{MHz}$ which can help process the high frequency input waveform. Similar to the C101HV pulse generator, the DS345 generator featured the 50 ohms BNC connectors which are compatible with the PICO sensors. The sampling rate of the arbitrary waveform can go up to $40 \mathrm{MHz}$. This means the time interval between plotted points can be as short as $0.025 \mu$ sec. Each arbitrary waveform could contain 16300 points. The DS345 can generate a waveform up to 10 volts. This may not be sufficient for long rods, especially under such high frequencies, as the attenuation would be much higher than the low frequency.

\section{Sensor and Preamplifiers}

The PICO sensors (made by the Physical Acoustics Corporation) were used in this study. The sensors are $4.2 \mathrm{~mm}$ in diameter, which is almost the same cross section size of the Grad 250 seven-wire strand's center wire. Each PICO sensor has its own calibration chart. According to the calibration chart, the response frequency is between $100 \mathrm{KHz}$ and 1 $\mathrm{MHz}$ and the average peak response frequency is about $500 \mathrm{KHz}$. 
The Physical Acoustics Corporation also produced an acoustic emission preamplifier with a model number of 1220A. This model is connected between the output sensor and the MISTRAS system. We have selected $40 \mathrm{~dB}$ as our gain level since the $60 \mathrm{~dB}$ gain level will be too noisy. The working frequency of the preamplifier is between $100 \mathrm{KHz}$ and $1.2 \mathrm{MHz}$.

Another type of sensor which is the S9208 was also used in this research. The S9208 is a displacement transducer with a flat frequency-response curve. This means that it can detect the wave front with more precision. On the other hand, the signal generated will be similar in comparison to the finite element results because the finite element results usually contain a displacement time history. The response frequency range of the S9208 displacement transducer is between $100 \mathrm{KHz}$ and $1 \mathrm{MHz}$.

\section{Data Acquisition System}

The MISTRAS (PAC) system is a fully digital, multi-channel, computerized acoustic emission system that performs waveform or signal measurement analyses. This system is also an IBM PC compatible. There are a total of 6 channels in this system and each channel can work simultaneously so we can monitor all channels at the same time. The sampling rate of the MISTRAS system can go up to $8 \mathrm{MHz}$ which will be important for our ultrasonic testing.

The Tektronix 2211 Oscilloscope can also be used to acquire data by means of detecting the waveform generated by the S9208 displacement transducer without bypassing the preamplifier. This advantage allows the oscilloscope to provide a clear waveform which translates to lower noise levels and a more accurate wave arrival time. The sampling rate of Tektronix 2211 Oscilloscope can goes up to $20 \mathrm{MHz}$ making the resolution time domain $0.05 \mu$ sec. $\left(5 * 10^{-8} \mathrm{sec}\right)$ 


\subsection{PROCEEDING OF THE EXPERIMENT}

There are two kinds of waveforms that were used in this study. One was generated by the DS345 function generator. The waveform contains 10 sine waves that were windowed by one half of a sine wave. The frequency of the sine waves are set to $308 \mathrm{KHz}$, $364 \mathrm{KHz}, 400 \mathrm{KHz}, 444 \mathrm{KHz}$, and 500KHz. Another input generator used was the C101HV pulse generator. Figure 3.1 showed the typical waveform on the receiving end of the short rod generated by the DS345 function generator. The input waveform was windowed by one half of a $500 \mathrm{KHz}$ sine wave. The input functions were shown in figures 3.2 to 3.6 which represent frequencies $308 \mathrm{KHz}, 364 \mathrm{KHz}, 400 \mathrm{KHz}, 444 \mathrm{KHz}$, and $500 \mathrm{KHz}$. Figure 3.7 shows the input function driven by pulse generator.

\subsubsection{Short Rod}

In the short rod experiment, the center wire of the seven-wire strand was used as a specimen. The wire is Grad 250 and the nominal diameter is $4.2 \mathrm{~mm} \mathrm{(3/16} \mathrm{inch} \mathrm{)} \mathrm{-} \mathrm{similar}$ to the diameter of the PICO sensors. For the widely used seven-wired strand, there are two common Grades: 250 and 270. In this experiment, we used a $158.8 \mathrm{~mm}$ (6 1/4 inch) long center wire in the seven-wire strand as our short rod specimen. The measured mass density is $7950 \mathrm{~kg} / \mathrm{m}^{3}$. The Young's modulus was assumed as $200 \mathrm{GPa}$, and the Poisson's ratio was assumed as 0.29 . 


\subsubsection{Long Rod}

Chen and Wissawapaisal (2002) have conducted several experiments for the 4.76 meter seven-wire strand. The seven-wire strand of Grad 250 was also used as a specimen in this study. The major difference between the two experiments is that a 3 meter center wire is used in this study as opposed to the 4.76 meter seven-wire strand used in Chen and Wissawapaisal's experiments. In the seven-wire strand, the helical wires make multiple contacts with the center wire. This leads to an increase in the attenuation and the distortion of the waveforms. However this study is similar to Chen and Wissawapaisal's study because the PICO sensors, used as transmitter and receiver, were also attached to the center wire during experimentation with the 4.76 meter rod. In this case, the output signals of the 4.76 meter rod and output signals from the other 2 shorter rods could still be comparable.

\subsubsection{Experimental Process}

Each PICO sensors were attached to both ends of the specimen. In order to receive a better wave motion, we needed an adhesive on the surface between the PICO sensors and the wires. There are two kinds of adhesives used in this experiment. One of the adhesives is the Ultrasonic Couplant Ultragel II made by Echo Ultrasound Company. The second type of adhesives used is Crazy Glue. Both of them can improve the output signal. In addition, the specimens should have less contact with other objects to reduce the unexpected distortion and energy dispersion along the rods.

After attaching the sensors on each end of the rod, we began by sending the pulses using the level of input; 30 volts for the C101HV pulse generator or 10 volts for the DS345 
function generator. The output wave signal can be detected almost at the same time by the Tektronix Oscilloscope 2211 system. After receiving the wave signal by using the oscilloscope, we transferred the data from a raw signal output to the ASCII code. The ASCII code is a common file format in modern computer systems making it easier to process and analyze.

\subsection{SHORT ROD EXPERIMENTAL RESULTS}

A typical signal on the short rod receiving end output can be seen on figure 3.1. There are several different frequency inputs that were gradually increased between $308 \mathrm{KHz}$ and $500 \mathrm{KHz}$. The output generated by the function generator ( $308 \mathrm{KHz}$ to $500 \mathrm{KHz}$ ) are shown from figure 3.8 to figure 3.12. The output generated by the C101HV pulse generator is shown on figure 3.13 .

\subsection{LONG ROD EXPERIMENTAL RESULTS}

Two long rods were used in the current long rod experiments. Chen and Wissawapaisal used the 4.76 meter seven-wire strand rod. One is the 4.76 meter rod which was accomplished by Chen and Wissawapaisal (2002). The other long rod is a single 3 meter wire strand taken from the seven-wire strand. The diameters of these two experiments are both $4.2 \mathrm{~mm}$ - the typical size of the single wire strand. The waveform of the pulse input driven by the C101HV pulse generator is presented in figure 3.14.

For the 3 meter single wire strand, the signal output of the $500 \mathrm{KHz}$ sine wave generated by the DS345 arbitrary function generator is shown on figure 3.15. In the 3 meter 
single wire strand, the amplitude of the waveform has more attenuation in comparison to the $158.75 \mathrm{~mm}$ single wire strand. In order to present the arrival time with more clarity, the 10X zoom-in on the vertical dimension (amplitude) is shown on figure 3.16 for the same waveform shown in figure 3.15. The C101HV pulse wave has also been sent in the 3 meter rod, and the output on the receiving end was shown in figure 3.17. It is obvious that in the figure 3.17, the wave arrival time is much more apparent than figure 3.15. That is because the amplitude generating performance is different between the arbitrary function generator and the pulse generator. The pulse generator could generate up to 30 volts at the peak of the pulse, but the function generator could only go up to 10 volts.

\subsection{EXPERIMENTAL RESULTS OBSERVATION AND DISCUSSION}

In the short rod experiments, we can see the waveform didn't disperse a lot after propagating on the short length center wire strand of $15.875 \mathrm{~cm}$. The output waveform and the input waveform remain the same. In the 3 meter or the 4.76 meter long rod, the waveform dispersion is more evident, this means the frequency components would separate more with the longer rods. In order to identify the frequency dispersion, longer rods are used to yield better results. Another obvious fact is when the wave propagates along the longer rods, there would be more attenuation occurs as a result. In Chen and Wissawapaisal's study, the pulses were sent to the 4.76 meter seven-wire strand. The peak voltage generated by the $\mathrm{C} 101 \mathrm{HV}$ pulse generator is about 375 volts. In addition, the output signal was powered by a preamplifier by about 100 times. Figure 3.14 shows that the peak signal on the receiving end is about 4 volts despite the fact that it has been enlarged 100 times $(40 \mathrm{~dB})$. In the case of the 3 meter single wire strand, the peak of the signal is 
about 0.015 volts which was detected by the S9208 displacement transducer without using the preamplifier. Another factor that can cause attenuation is the contacts on the rods or the sensors. Contacts on the rods could cause wave attenuation and wave distortion. If the contacts happened on the sensors or the pulsers, they could cause an abnormal motion in the sensors or pulsers which would ultimately affect the amplitude of the wave. In the case of the 4.76 meter seven-wire strand, it is impossible to avoid the contacts between the center wire and the helical strands. In order to detect an accurate and clear signal, we should decrease the number of contacts to least possible.

Figure 3.15 and 3.16 show the wave signal of the same setup on the 3 meter rod. The same setup means the input, rod, pulser and sensor are same. It is obvious that figure 3.15 doesn't present the arrival time well enough due to the amplitude resolution. Figure 3.16 shows the enlargement with the 10 times zoom-in on the voltage axis. Even when the noise has also been enlarged, we can still identify the arrival time because the arrival time is an important component to understand the material properties. Using the same 3 meter signal wire strand, figure 3.17 shows the output wave signal generated by the pulse generator and detected by the S9208 displacement transducer which shows a very clear arrival.

\subsection{SUMMARY}

In this chapter, we have discussed three different specimens and two kinds of input waveforms which presented in the figures. The experimental result indicated that the longer rod is better for observing the dispersion of the frequency components. However, longer rods presented some limitations such as energy attenuation and wave distortion. The short rod shows the arrival part of a waveform clearly, but it is not long enough to show the 
frequency separation. In this study, all the specimens and the testing resulting are under zero tensile forces in order to compare with the Algor simulation result in the next chapter. 


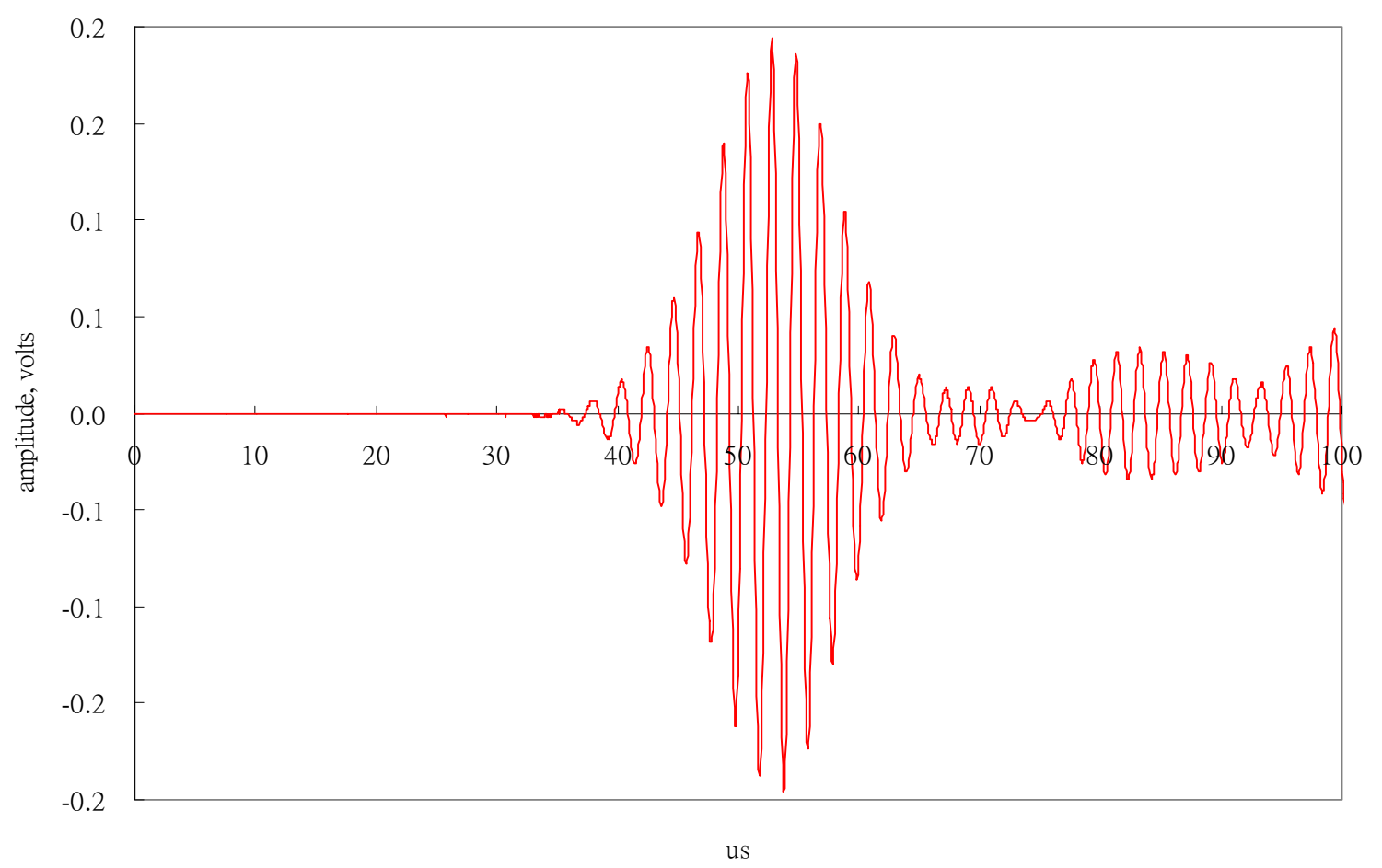

Figure 3.1 The typical waveform at the receiving end of the rod.

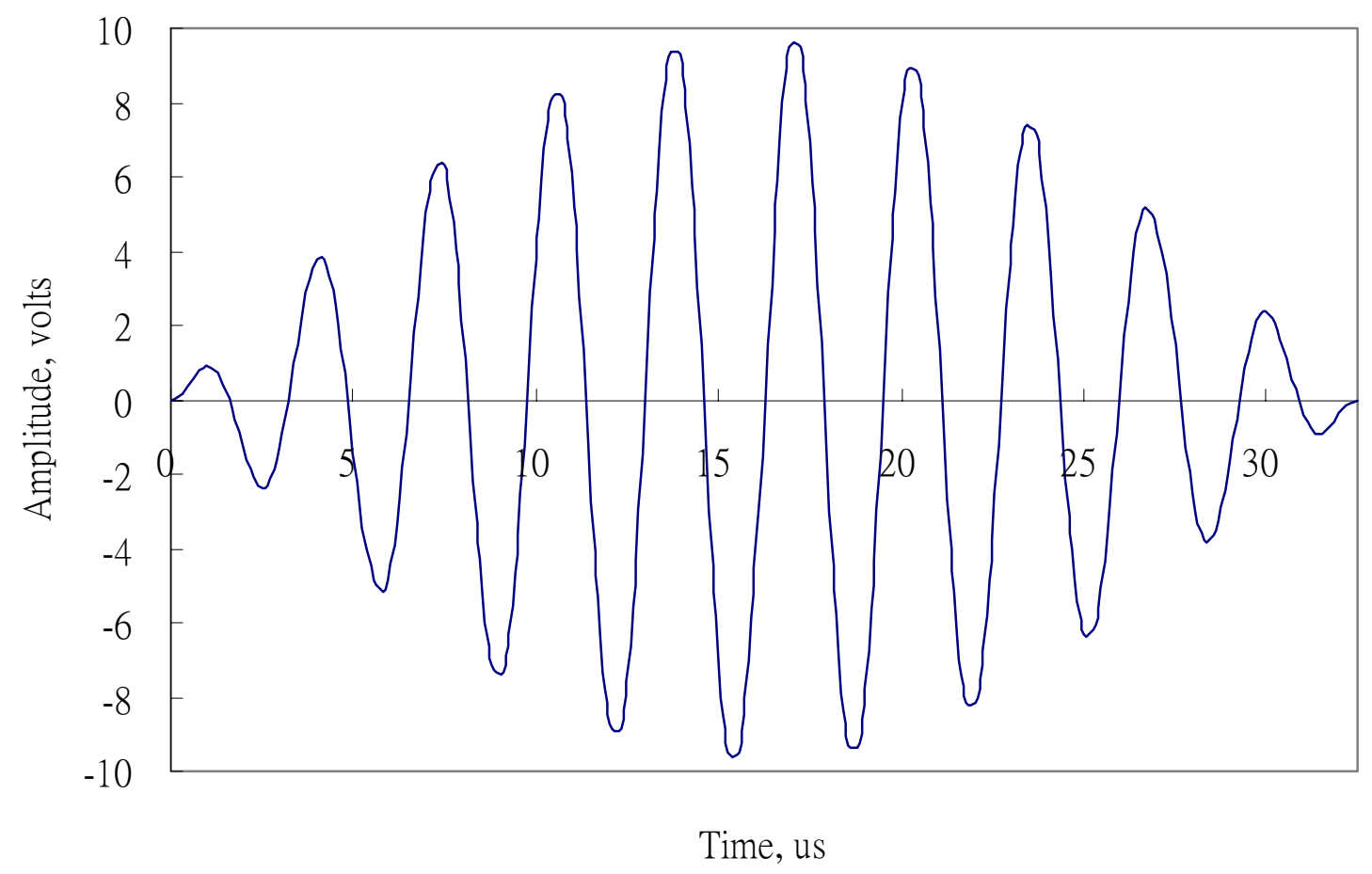

Figure 3.2 The input of $308 \mathrm{kHz}$ sine wave 


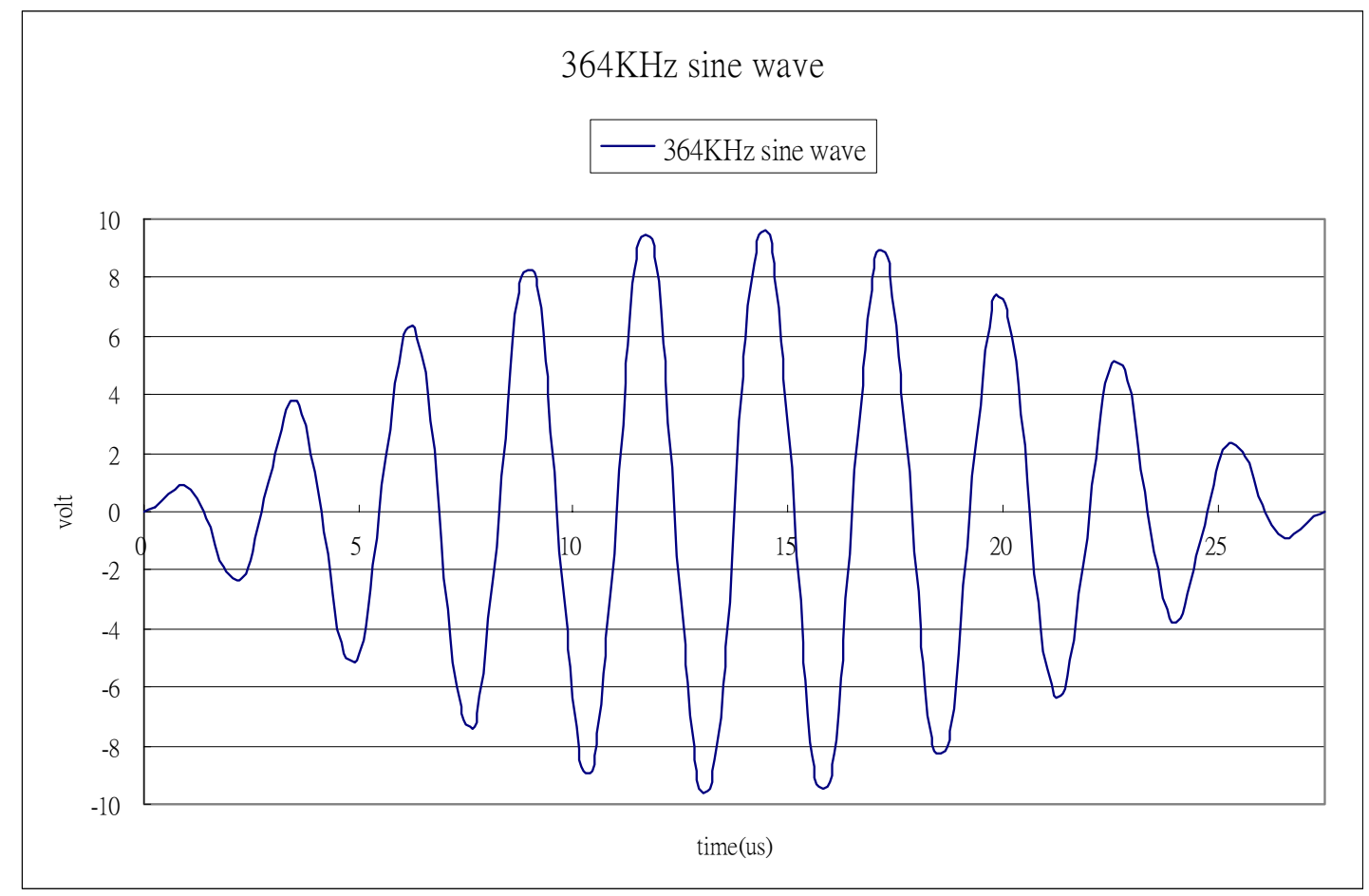

Figure 3.3 The input of $364 \mathrm{kHz}$ sine wave

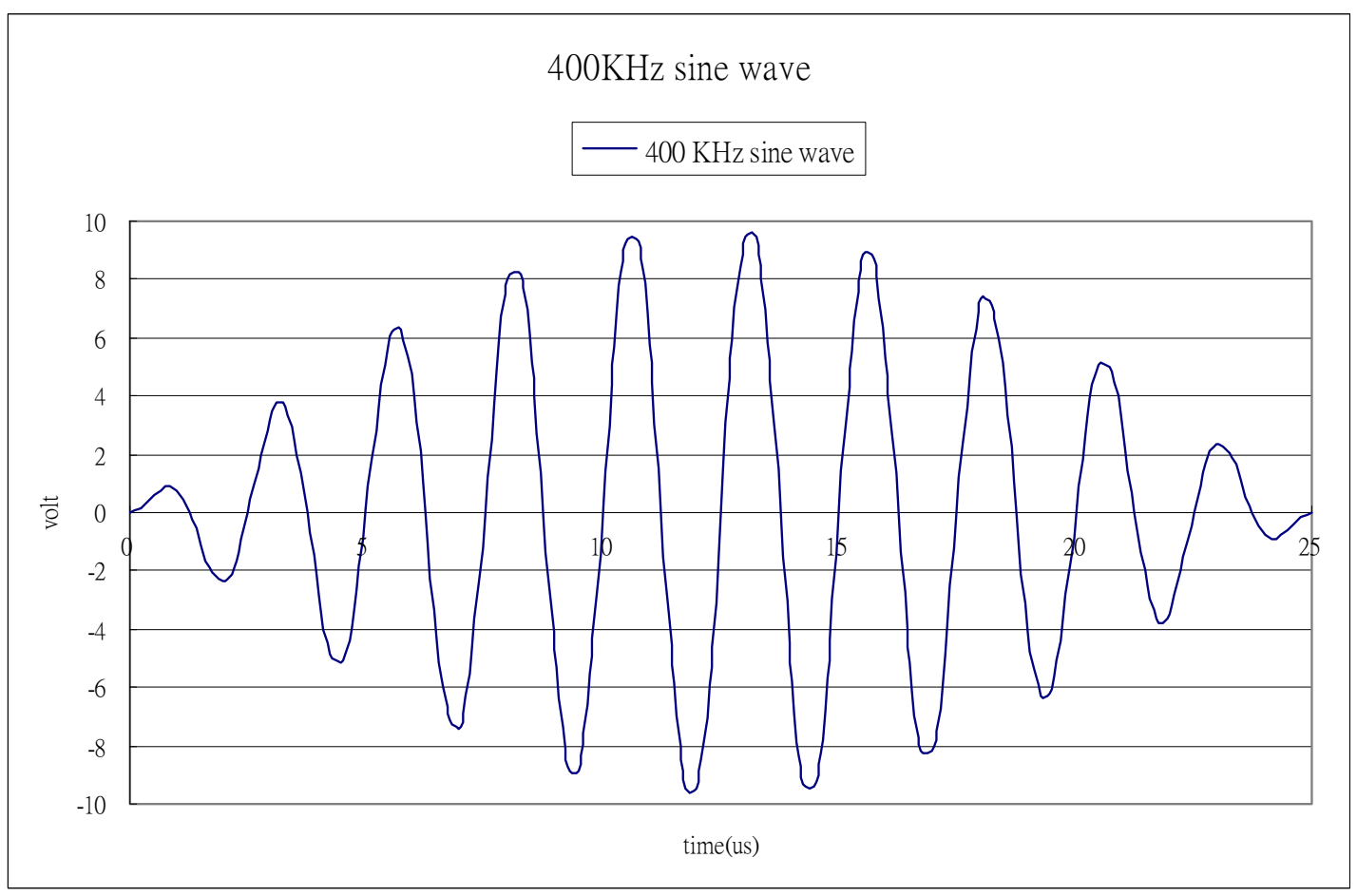

Figure 3.4 The input of $400 \mathrm{kHz}$ sine wave 


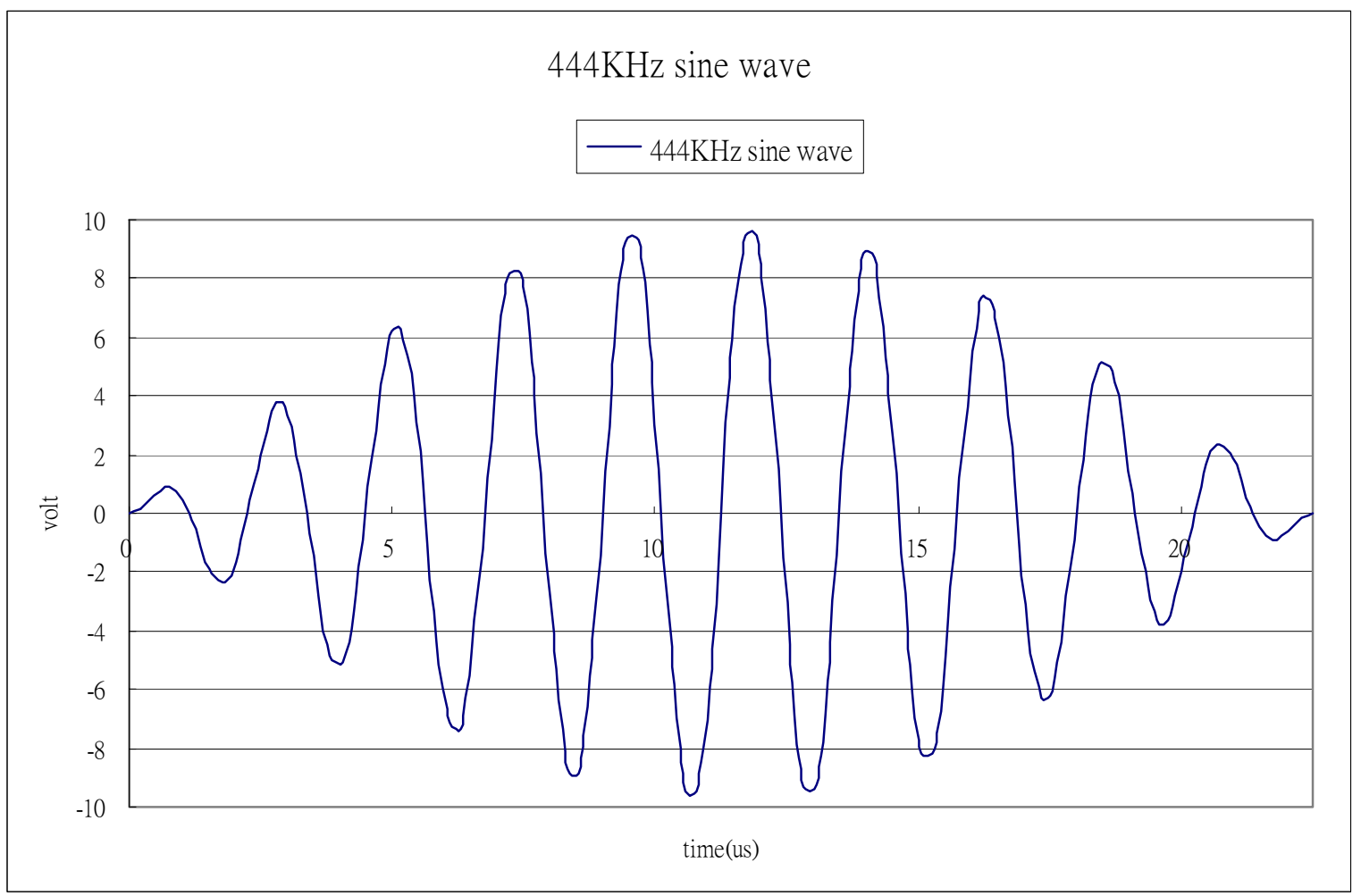

Figure 3.5 The input of $444 \mathrm{kHz}$ sine wave

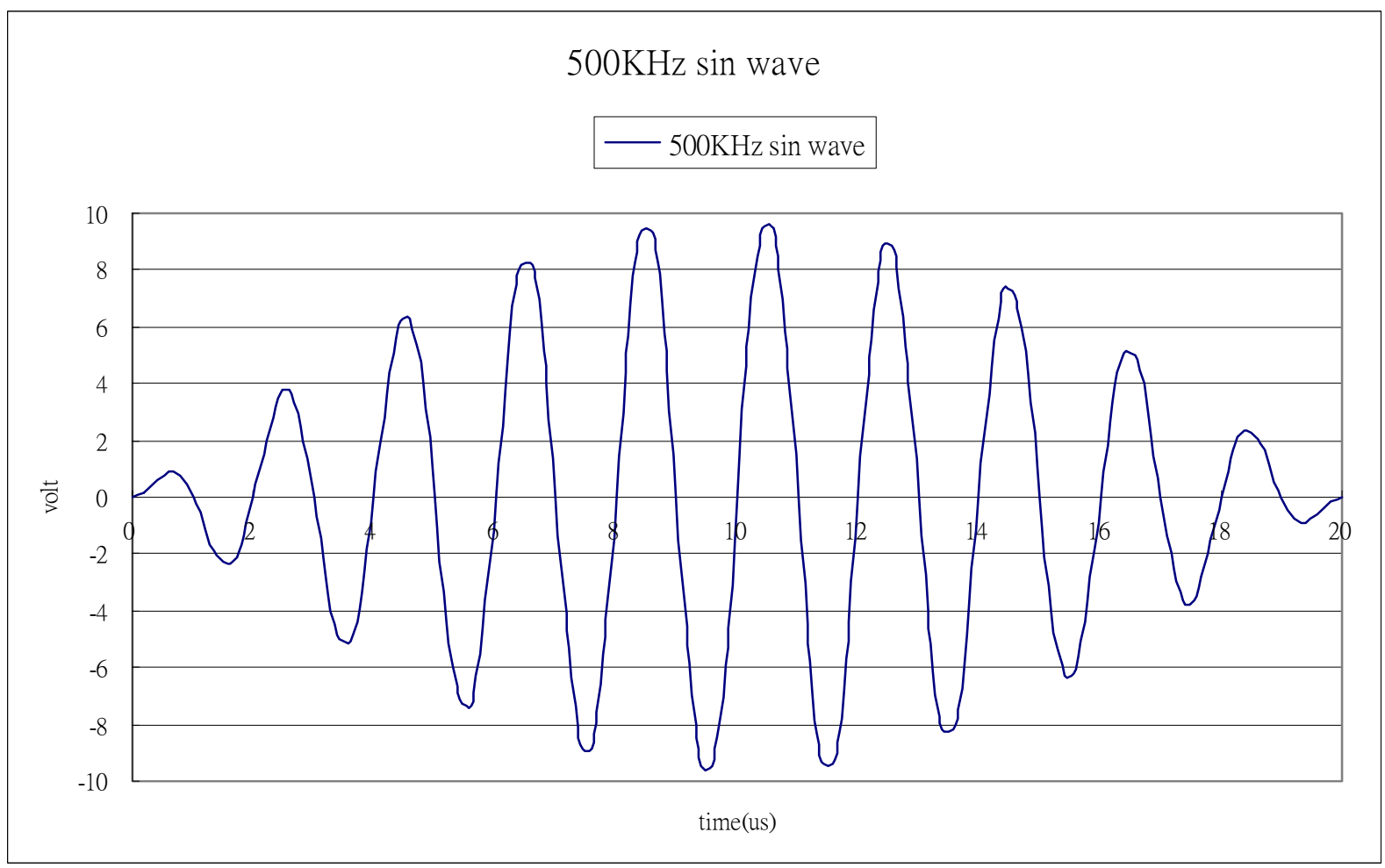

Figure 3.6 The input of $500 \mathrm{kHz}$ sine wave. 


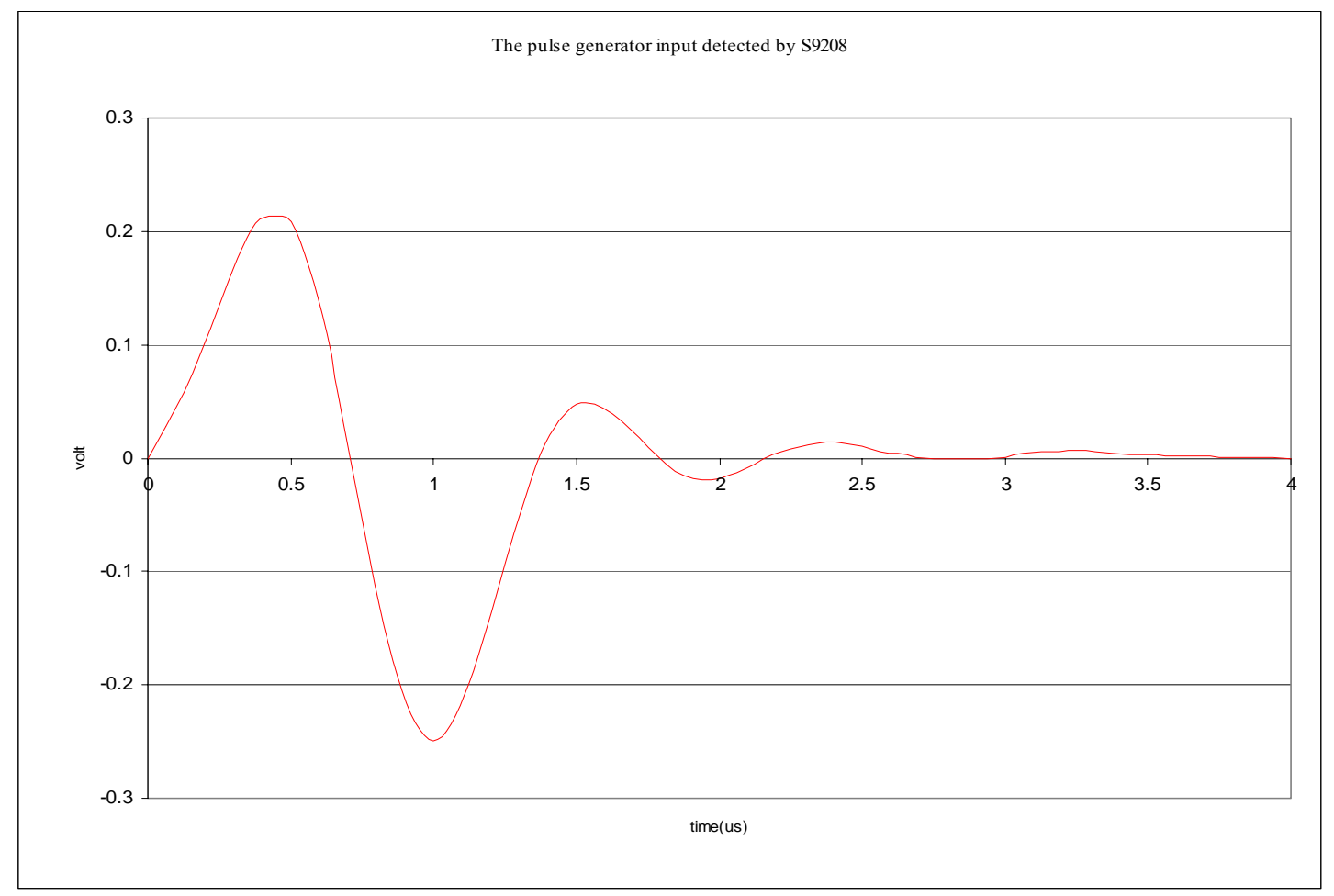

Figure 3.7 The input of the pulse generator

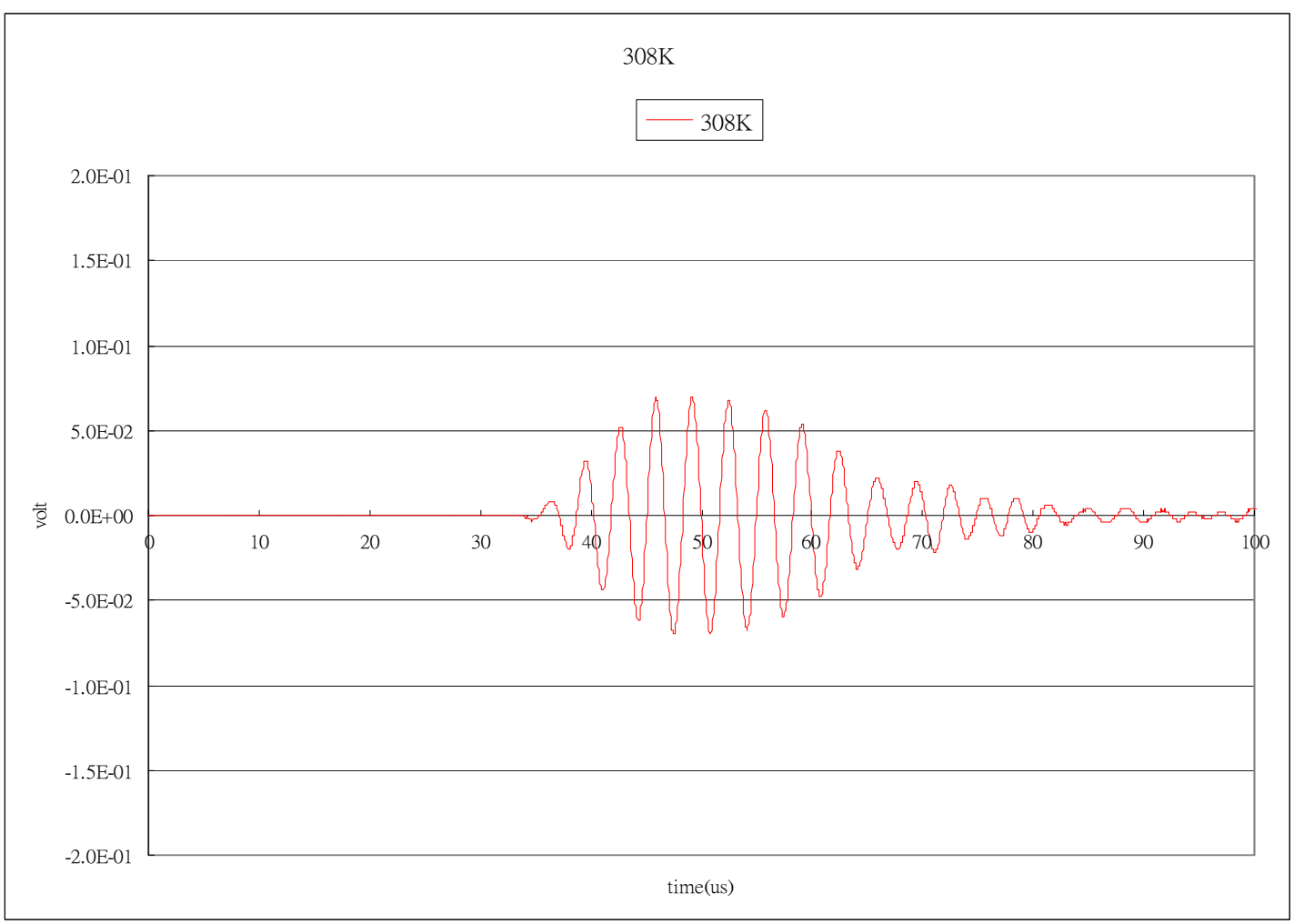

Figure 3.8 The $308 \mathrm{kHz}$ sine wave output of the short rod 


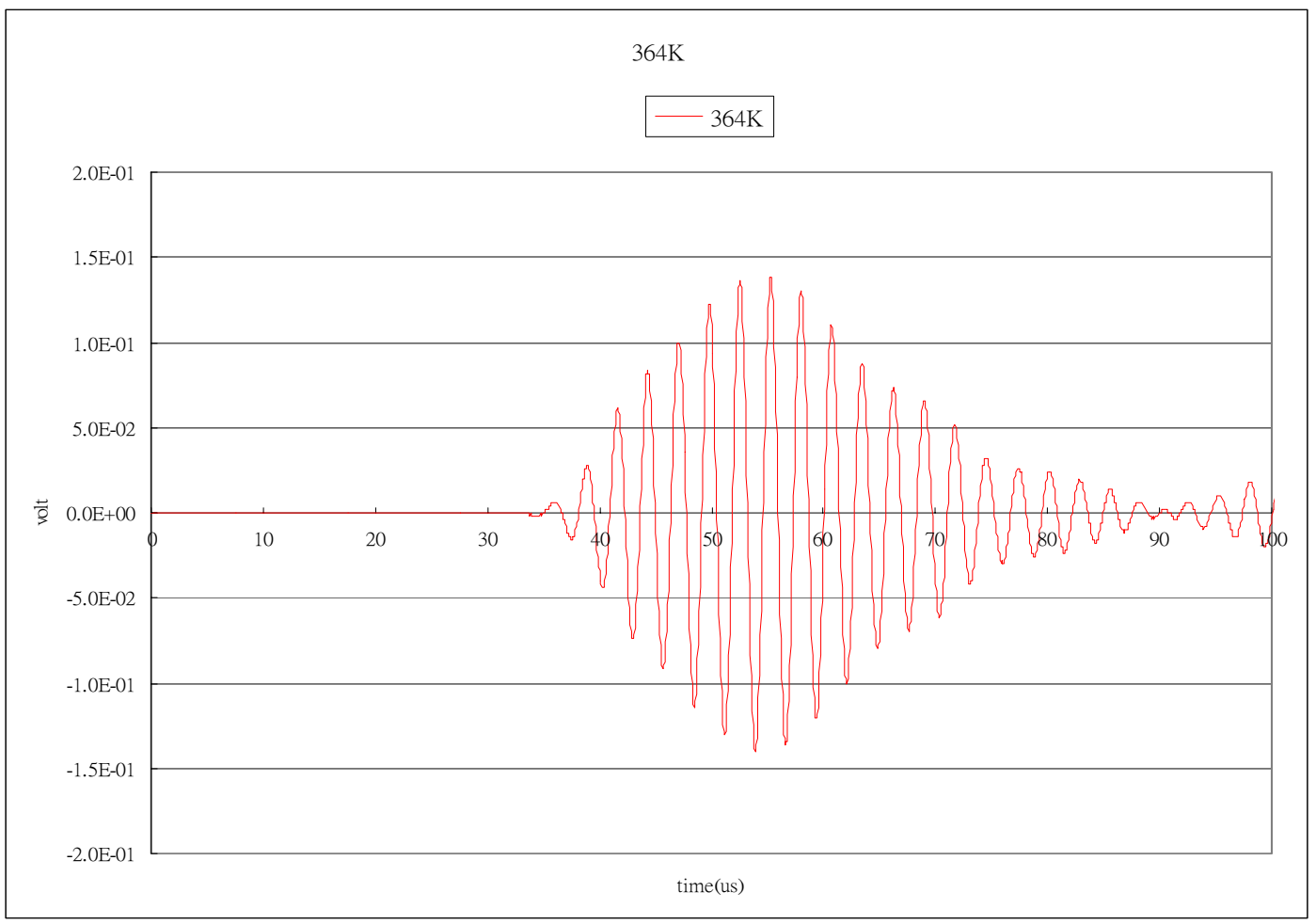

Figure 3.9 The $364 \mathrm{kHz}$ sine wave output of the short rod

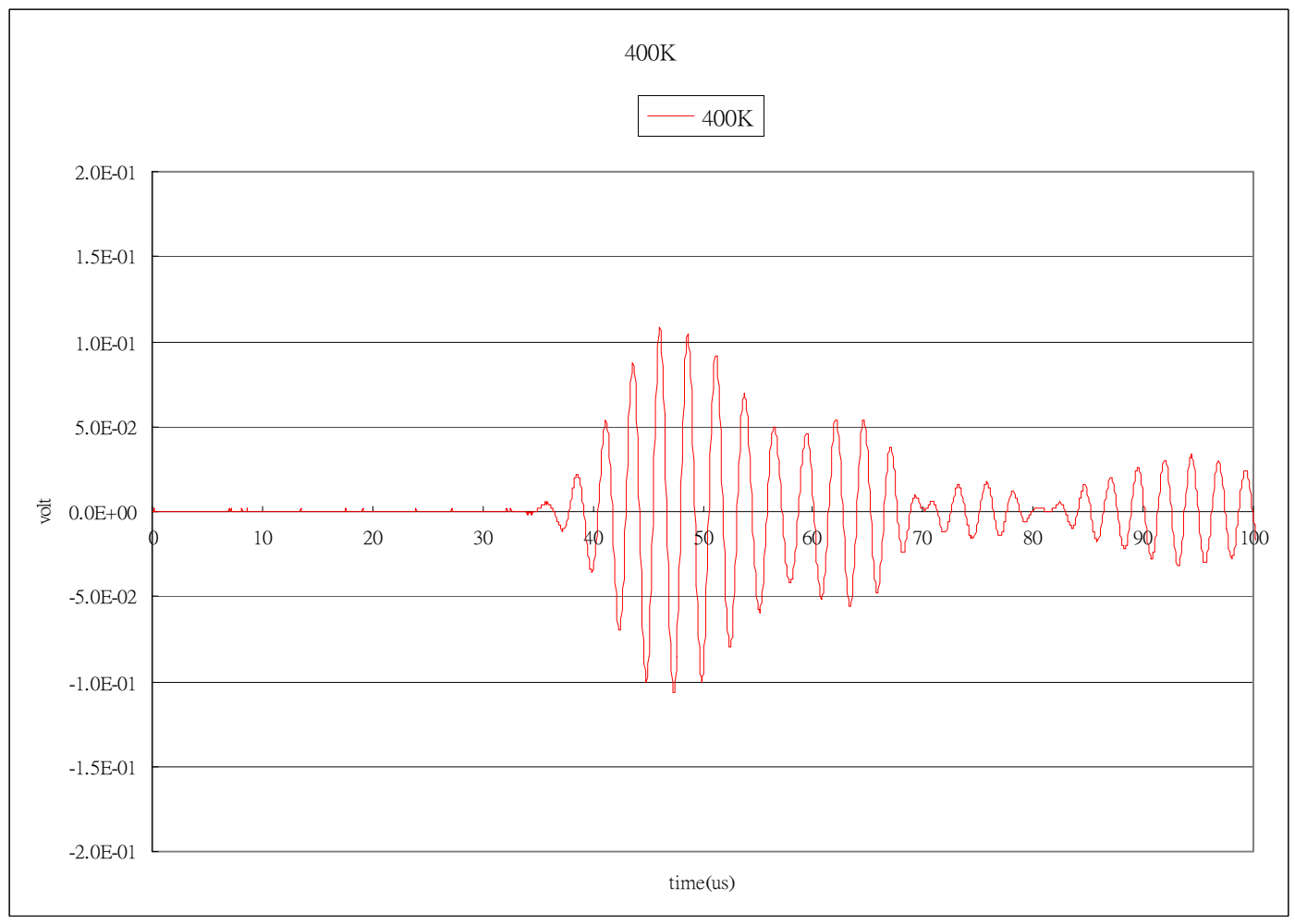

Figure 3.10 The $400 \mathrm{kHz}$ sine wave output of the short rod 


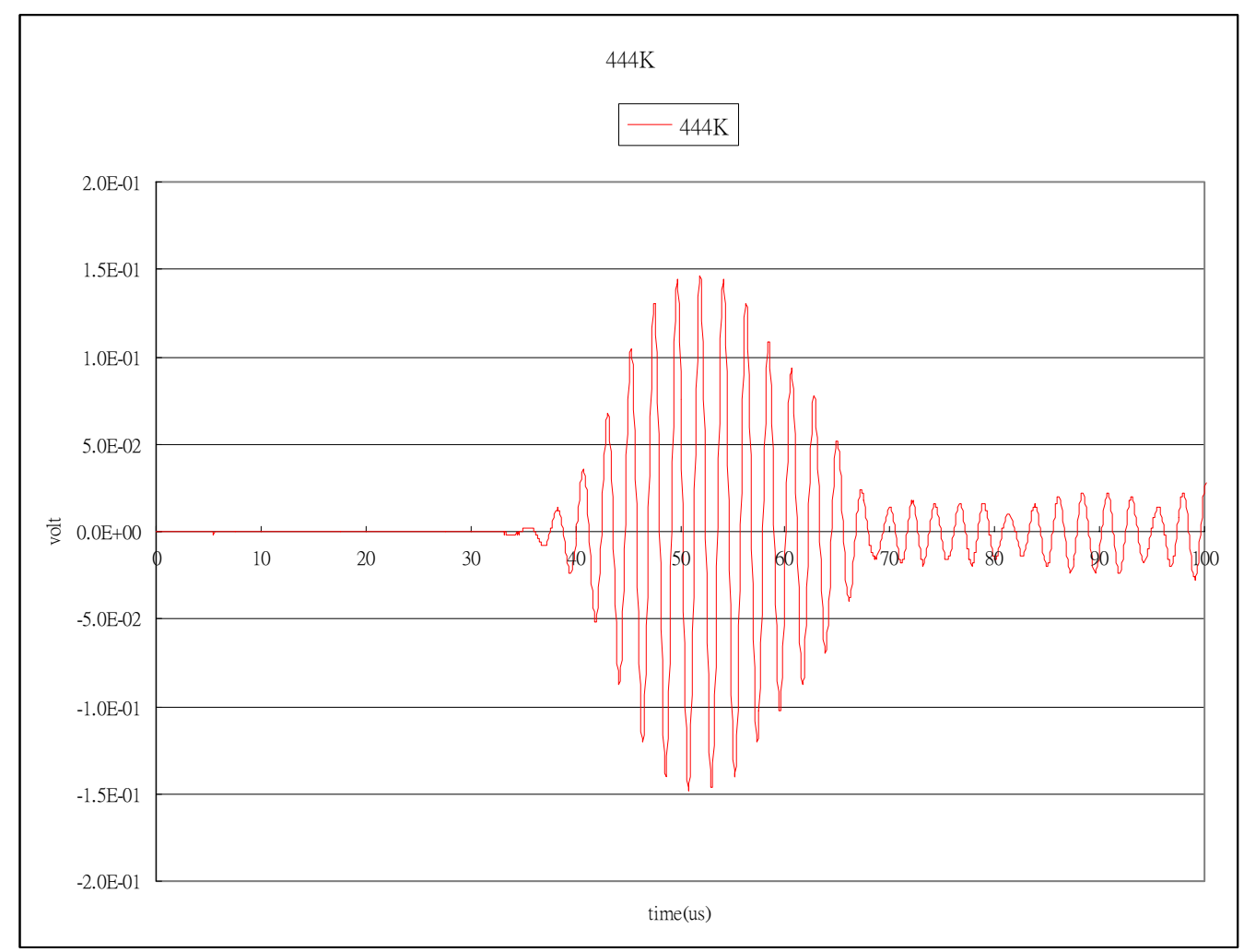

Figure 3.11 The $444 \mathrm{kHz}$ sine wave output of the short rod

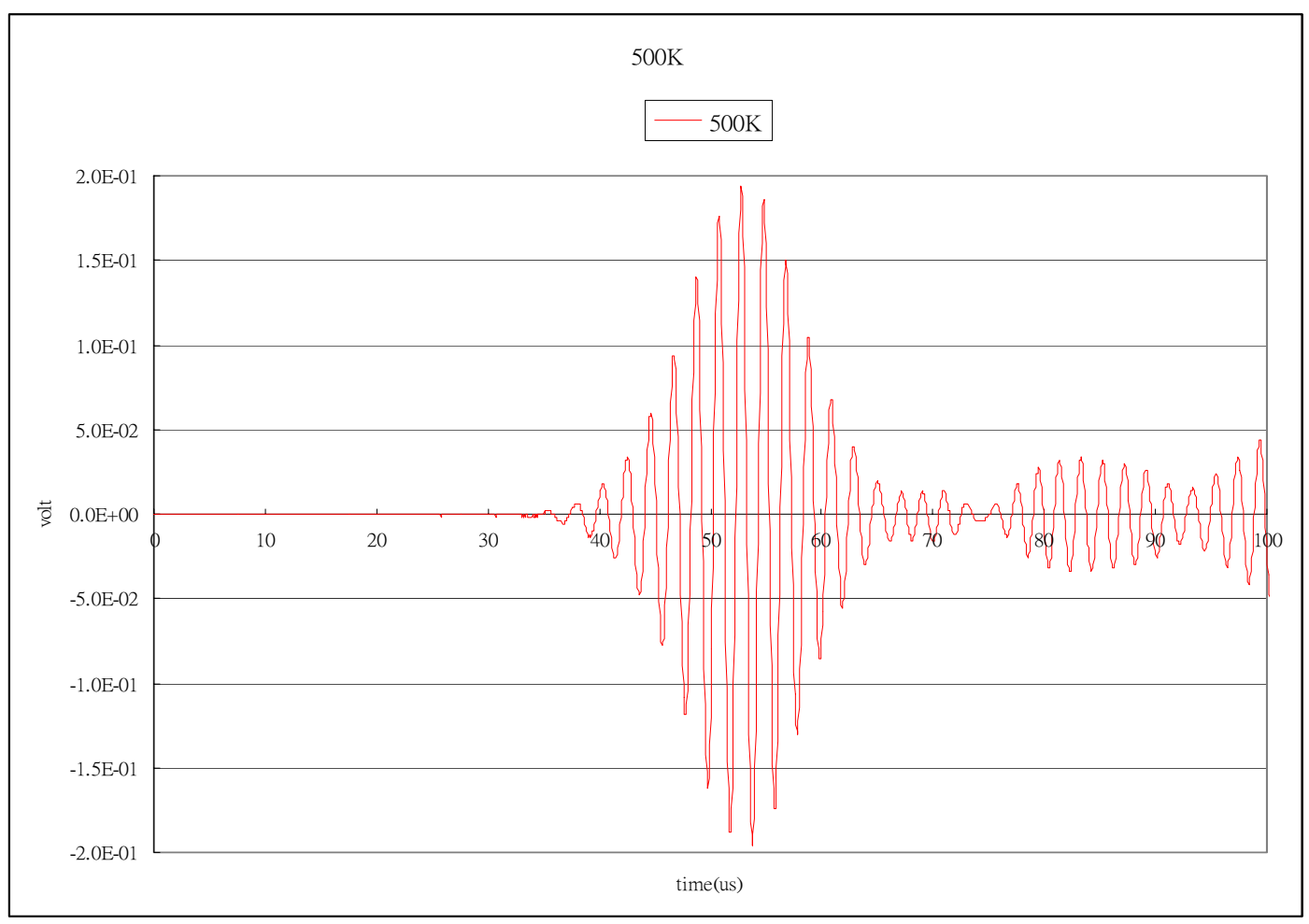

Figure 3.12 The $500 \mathrm{kHz}$ sine wave output of the short rod 


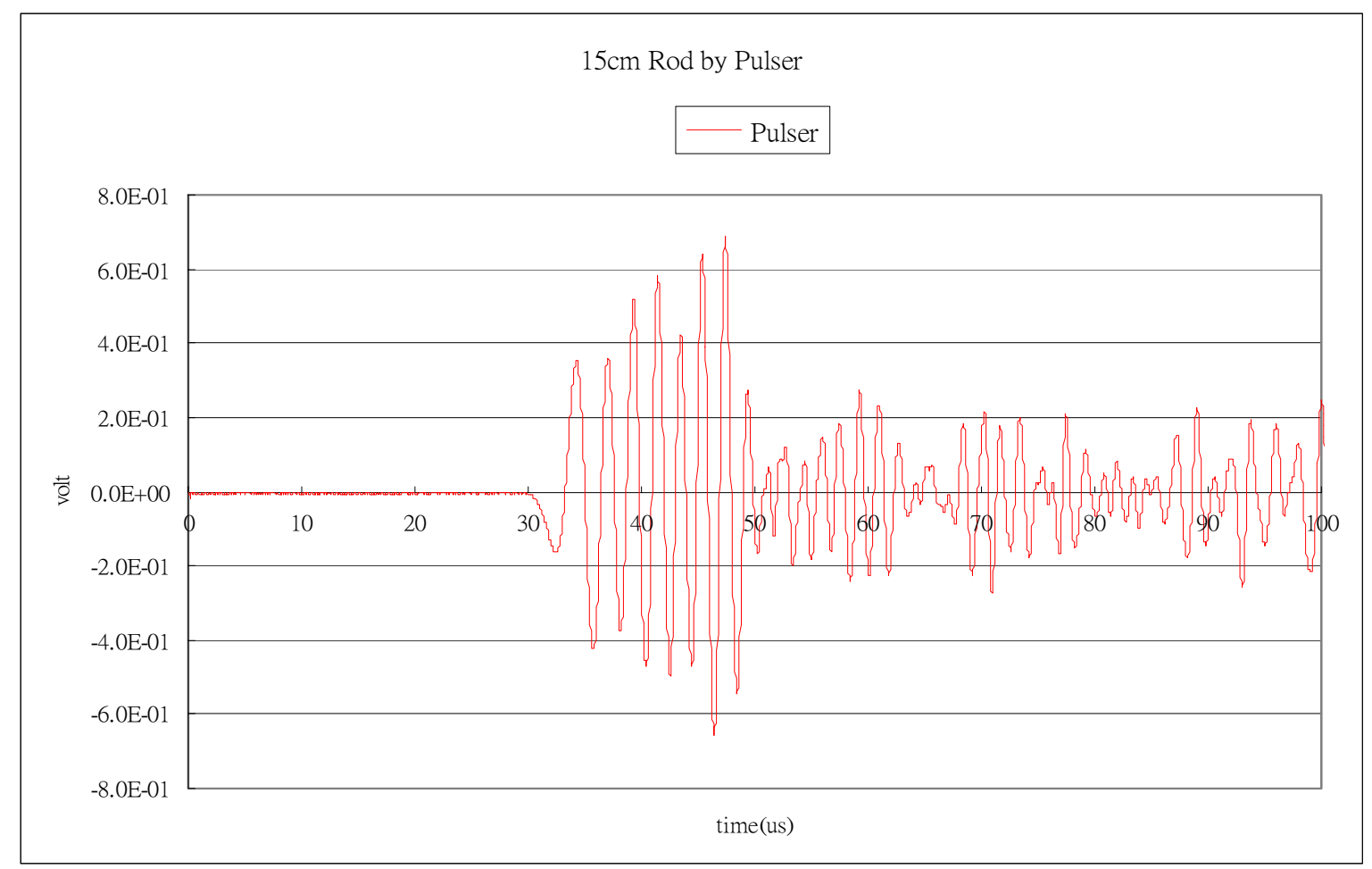

Figure 3.13 The output generated by $\mathrm{C} 101 \mathrm{HV}$ on the short rod

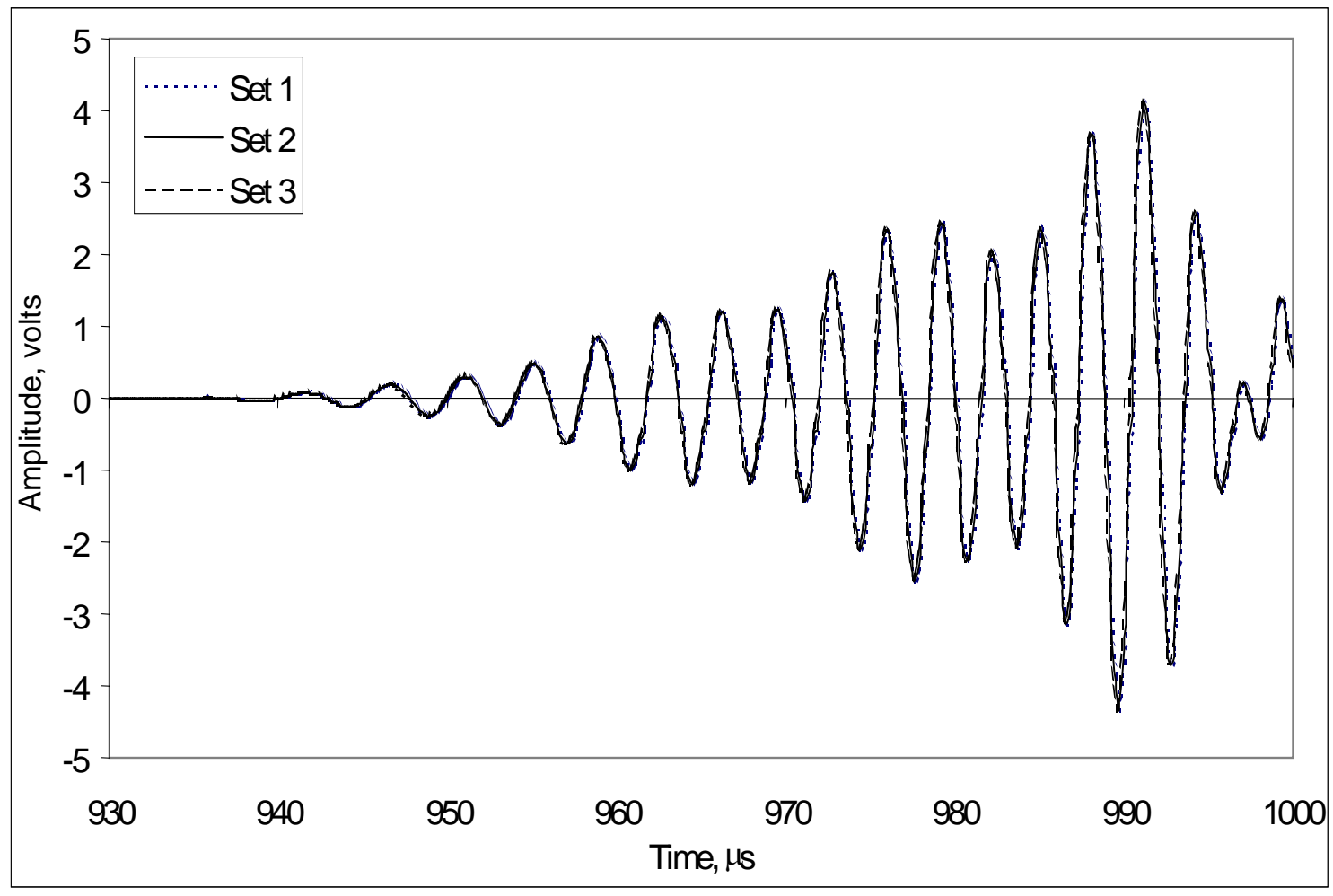

Figure 3.14 The output generated by $\mathrm{C} 101 \mathrm{HV}$ pulse generator on $4.76 \mathrm{~m}$ long rod (Chen and Wissawapaisal, 2001) 


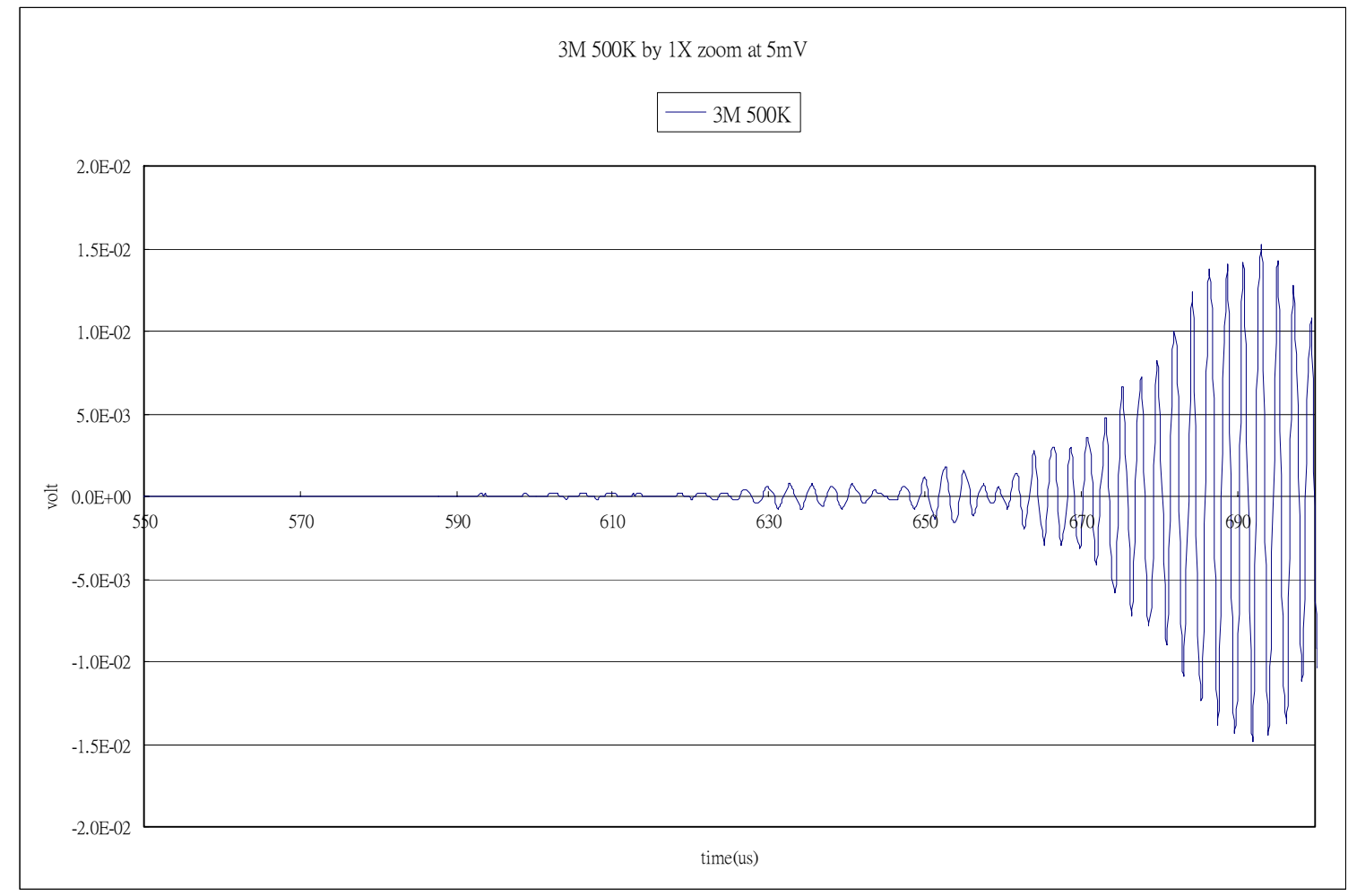

Figure 3.15 The $500 \mathrm{kHz}$ sine wave output of the 3 meter single strand

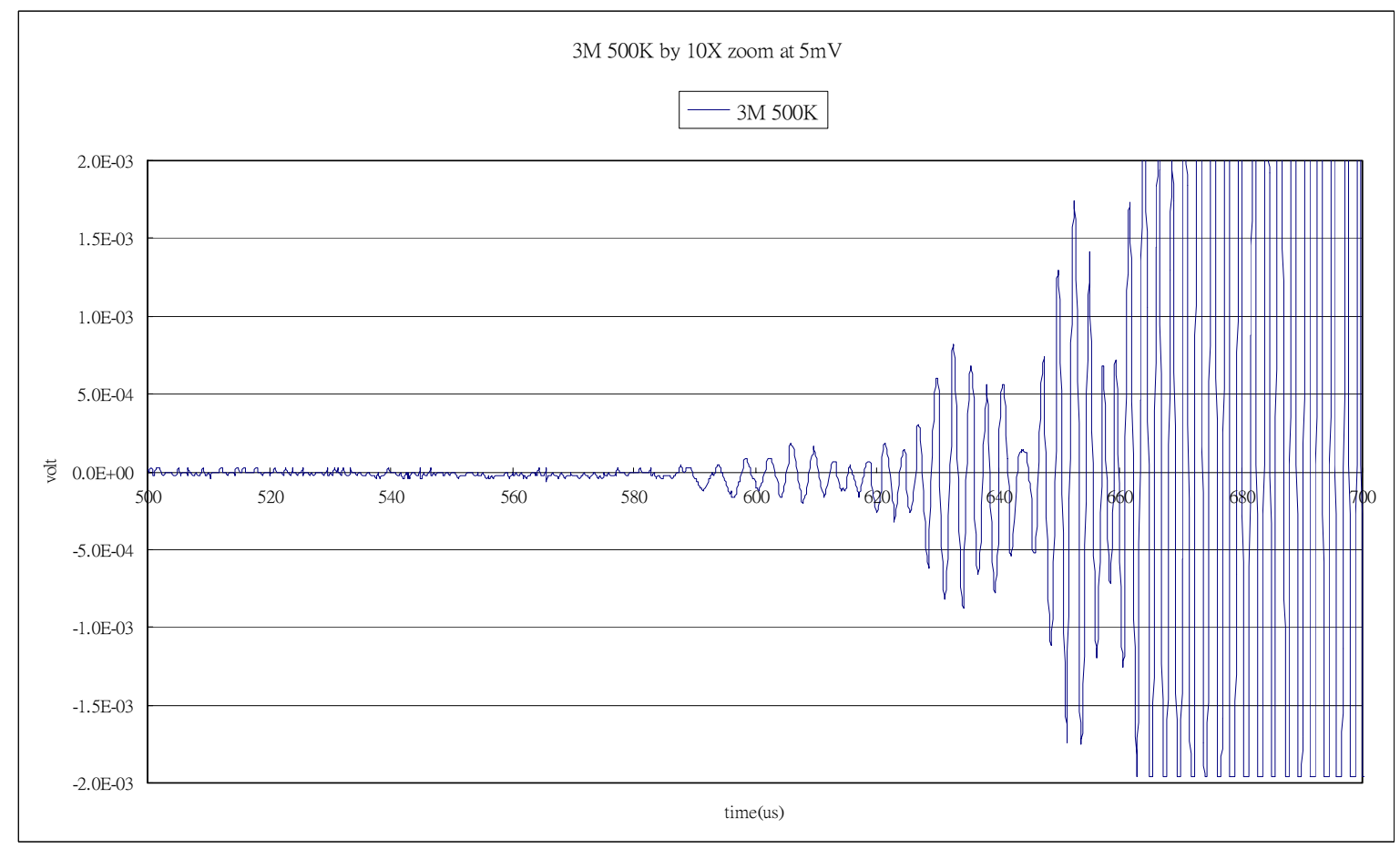

Figure 3.16 The zoom in of the 5.15 (present the wave arrival time with more clarity) 


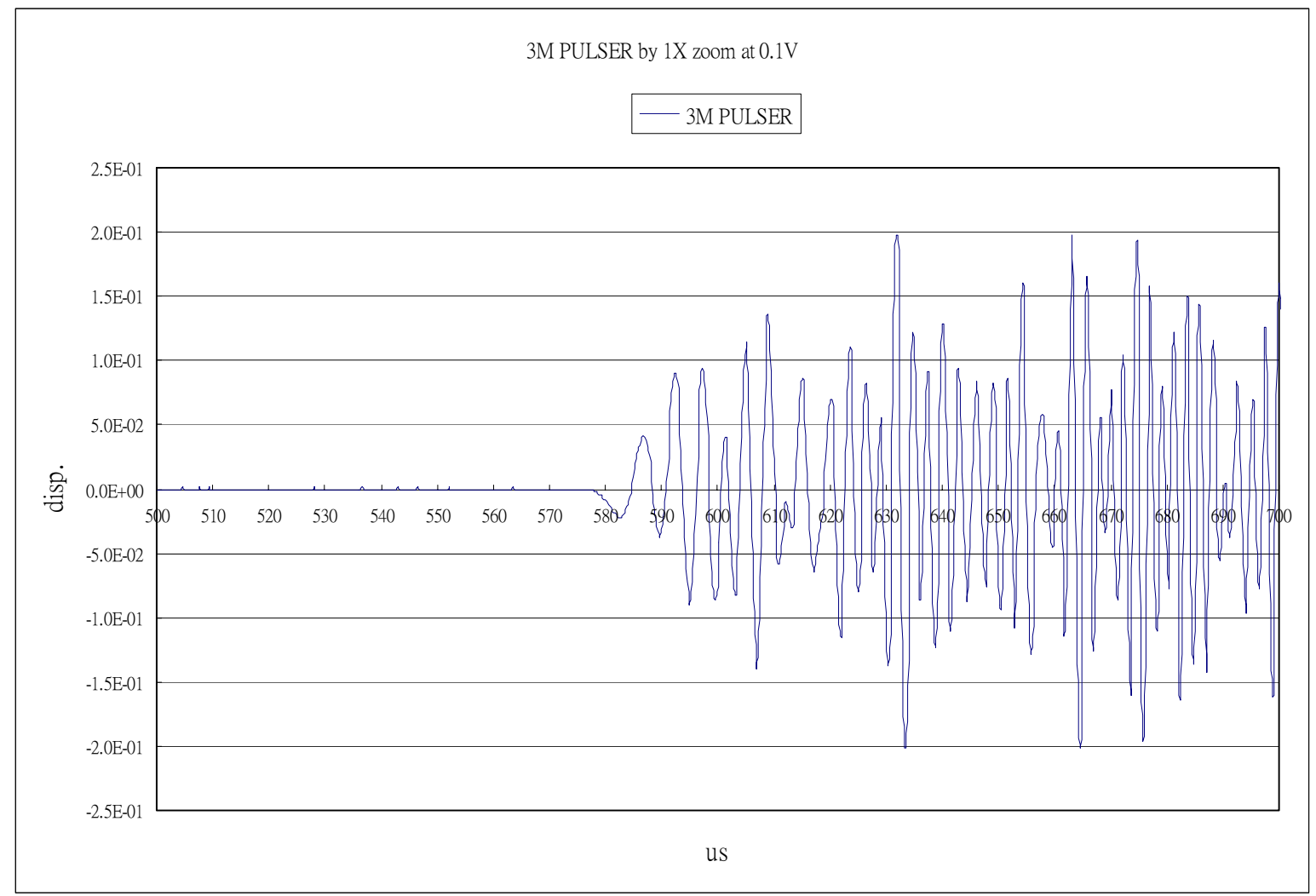

Figure 3.17 The output wave signal generated by pulse generator recorded on the receiving end of the 3 meter rod 


\section{CHAPTER 4}

\section{THE APPLICATION OF FINITE ELEMENT METHOD FOR ULTRASONIC WAVE PROPAGATION IN A SOLID ROD}

\subsection{OVERVIEW}

The Finite Element Method (FEM) is a popular method for providing numerical solutions. In order to make the solution comparable with experimental results or theoretical solutions, it is important to determinate the factors in the simulation. The element size and material properties of the simulation model must be considered. Algor software is used as a FEM computer simulation program in this study. With this software, two kinds of models were generated and will be presented. One is a three-dimensional (3D) model and the other is an Axis-Symmetric model. In order to make sure the FEM works correctly for wave propagation in a solid rod, we will compare the experimental results and theoretical solutions in the next chapter.

\subsection{INTRODUCTION OF FEM SOFTWARE}

Algor is a well-established finite element program for the computer simulation of static and time-dependent dynamic analyses. In this study, the dynamic linear analysis is used for the wave propagation problems. 


\subsubsection{3-D (Brick) Model}

To simulate any real-life object in Algor, we can just simply generate a model that has the same appearance of the object. Then we put the geometric mesh on the model. The procedure of generating the 3-D rod will be presented in the following paragraph.

First of all, there are two essential dimensions for any rod. One is the radius (r) and another one is the length $(\mathrm{L})$. On the $\mathrm{Y}-\mathrm{Z}$ plane, set four points as $(0,0),(\mathrm{r}, 0),(\mathrm{r}, \mathrm{L})$, and $(\mathrm{L}, 0)$. Then link these four points two by two in order to form a rectangular plate on the Y-Z plane. After generating the rectangular plate, we start to put the geometric mesh on it. The number of the elements along the Y-axis will decide how many layers there will be along the radius of this rod. The number of the elements along the Z-axis will decide how many divisions there are along the length of this rod. After adding the geometric mesh into the rectangular plate, we can begin to rotate the meshed-rectangular plate. In order to form a cylindrical rod, the rectangular plate should be rotated 360 degrees along the z-axis (the line between $(0,0)$ and $(0, \mathrm{~L}))$. The number of degrees that the rectangular is rotated each time is relative to how many sides of the rod cross section there will be. For example, if we rotated 60 degree each time, after we did 6 times, a hexagonal cross section will be formed. By rotating 30 degree each time, we will get a dodecagonal (12-side) cross section. In General, the cross section shape should be generated as close to a circle as possible. This means more sides for the cross section will be better. In addition, the element number of a 12-sided cross-sectional model will be twice that of the 6-sides model. Due to computer limitations such as hard drive space, the speed of processor and the size of system memory, too many elements will cause delays in the simulation. This is because detailed finite

element models have huge numbers of elements. Although more elements usually provide 
better result, we also need enough hard drive space and time to accomplish this model. Especially in the long rod simulations, the waves will consume more time to propagate the whole length of the rod. That means more time steps will be involved in the simulation. In order to solve these problems, the axis-symmetric model could be an efficient answer for simulating the wave propagation in solid rods. The details of the axis-symmetric model will be described in the next section.

\subsubsection{Axis-Symmetric Model}

Axis-symmetric FEM models have to be symmetric along at least one axis. The rods we used in this study satisfy this requirement because the cylindrical bars are symmetric along the center line of the cross section. To generate the axis-symmetric model, the same steps as for the 3-D model are followed until generation of the geometric mesh. After generating the FEM mesh on the rectangular plate, we don't have to rotate the plate along the Z-axis. This is because the sub-programs of the axis-symmetric model and the 3-D model are different. The sub-program of the axis-symmetric model will rotate the rectangular along the Z-axis automatically. Due to this advantage, the cross section of the model will be a perfect circle.

\subsection{COMPARSION BETWEEN 3-D AND AXIS-SYMMETRIC MODEL}

The major difference between 3-D and axis-symmetric models is the number of elements. For example, two kinds of model have the same rectangular plate with the same number of elements on it. In the 3-D model generating procedure, we have to rotate the 
rectangular plate several times in order to form a cylindrical bar. This rotation step also duplicates the number of the elements on the rectangular plate. For instance, if there are 10,000 elements on the rectangular plate, after rotating six times, the number of the elements will be enlarged to 60,000 .

In addition, the cross section of the 3-D models is still not a perfect circle. Figure 4.1 shows the cross-sectional difference between the 3-D model and the Axis-symmetric model. This means the cross section could be a 6-side or 12-side polygon with sharp angles. The sharp angles will make unstable reflections occur which could distort the output waveform.

While generating the axis-symmetric model, the rotation step of the rectangular plate is unnecessary. In addition, the sub-program of the axis-symmetric will do the rotation for the rectangular plate without increasing the number of the elements. This means that there are at least two advantages of not having to rotate the rectangular plate, which are:

1. After forming the cylinder bar, the number of the elements still remains the same.

2. The Algor program will consider the cross section as a perfect circle.

Even though modern computers can handle huge numbers of elements, sometimes they are still not efficient enough to accomplish ultrasonic wave propagation problems. Especially for the longer rods, the calculation of the ultrasonic wave propagation usually challenges modern personal computers. Because ultrasonic waves have high frequencies and short wave lengths, in order to present the ultrasonic wave propagation in the solid rod, tiny element sizes would have to be used in the model. Otherwise the output will not be accurate. Generally speaking, if we want to simulate the 5 meter long rod, it would take about more than two days to finish the FEM simulation if we were using the 3-D model (on a 2.5 GHz processor computer). But if the axis-symmetric is used for the same case, it will 
take less than 24 hours.

It is obvious that the axis-symmetric model is better than the 3-D model for simulating ultrasonic waves. Therefore, most of the following FEM outputs were generated by employing the axis-symmetric models.

\subsection{THE DETERMINATION OF LOADING FUNCTION, LOADING FREQUENCY, ELEMENT SIZE AND TIME STEP}

\subsubsection{The Frequency Components of the Input Loading Functions}

Each input waveform has its own frequency component. For the sine wave input loading, the frequencies are $308 \mathrm{kHz}, 364 \mathrm{kHz}, 400 \mathrm{kHz}, 444 \mathrm{kHz}$, and $500 \mathrm{kHz}$. For the waveform generated by the $\mathrm{C} 101 \mathrm{HV}$ pulse generator, the frequency distributes between $100 \mathrm{kHz}$ and 1.5MHz. The peak of this frequency distribution falls on $750 \mathrm{kHz}$. The spectrum curve of the C101HV pulse is like a bell-shaped distribution. The frequency component is important because different frequencies propagate with different group velocities. Figure 4.2 shows a schematic figure of the FEM loading input located at the center of the cross section.

Figure 4.3 shows a frequency-group velocity chart of a common Grad 250 signal wire strand $\left(\varnothing=4.2 \mathrm{~mm}, \rho=7800 \mathrm{Kg} / \mathrm{m}^{3}, \mathrm{E}=211 \mathrm{Gpa}, \nu=0.2695\right.$, by Chen and Wissawapaisal 2002). It presents the group velocity curves of the first two longitudinal modes. This curve is also affected by the diameter of the rod. In this Grad 250 seven wire strand case, the highest velocity of the second mode is about $4300 \mathrm{~m} / \mathrm{s}$, and the same speed in the first mode occurs at $500 \mathrm{kHz}$. This means if the input waves with the first mode frequency are higher than $500 \mathrm{kHz}$, the second mode component will also be involved in the propagation. 
The second mode waveforms are highly distorted and unstable, which are shortcomings for the observation.

In real experiments, in order to describe the separation of certain frequencies after the input waves propagate a long distance, we need to use the DS-345 arbitrary function generator for generating the waves at given frequencies. In FEM simulations, the input waveform could be easily imported by using Microsoft Excel. The FEM inputs have to be exactly same as the experimental inputs. For example, Figure 4.4 shows 10 continuous sine waves at $500 \mathrm{KHz}$, and these 10 sine waves are multiplied by a half-sine wave. The sine wave inputs of the other frequencies have been shown in Figure 3.2 to 3.6.

Just like the experimental inputs, besides the DS345 arbitrary function generator inputs, another kind of input was also used in this study. It is a short period pulse which is generated by the $\mathrm{C} 101 \mathrm{HV}$ pulse generator. This input is also used in FEM simulations. Figure 4.3 shows the input which is exactly the same as the C101HV pulse generator input. For such a high frequency pulse input, the second mode longitudinal wave will propagate as fast as the first mode waves. This means the output wave front will exhibit the effects of the second mode waves.

\subsubsection{The Element Size}

The term element size in this section will refer to the element length along the length of the model (rod). In order to determine the right element size, the frequency of the input waves must be considered. The basic concept is that the element length must be shorter than the wave length. To solve the wave propagation problems by employing FEM, each element length should be at least three times smaller than the wave length. In other words, 
each wave length should contain at least 3 elements. For better resolution, the element size sould be made as small as possible. For Example, take " $\mathrm{f}$ " as the frequency of a input wave. The frequency " $\mathrm{f}$ " could be transferred to radius frequency by Eq. 4.1

$$
2 \pi f=w
$$

where the unit of $\mathrm{w}$ is radius/sec, and the bar velocity is assumed as $\mathrm{C}_{\mathrm{b}}$. Then the wave length $\lambda$ is derived by Eq 4.2

$$
\lambda=C_{b} / w
$$

After calculating wave length $(\lambda)$, we can start to determine how many elements $(\mathrm{N})$ should be contained by one wave length $(\lambda)$. The element size $L_{e}$ can be obtained by Eq 4.3

$$
L_{e}=\lambda / N
$$

In case of short strand model, $\mathrm{N}=10$ could be taken for better resolution. For long rod, $\mathrm{N}$ could be reduced to 5 or even 4 , which gives bigger element size. However, $\mathrm{N}$ must be restricted to equal or higher than 3. Even though a larger $\mathrm{N}$ is better, it is also important to consider the processing time and the hard drive space. For example, if we chose $\mathrm{N}=10$ in the long rod simulation, an extremely large number of elements is needed, therefore the delay of calculation would happen in the simulation. In addition, too many elements will consume a lot of hard drive space. 


\subsubsection{The Integration Time Step}

The time step $\Delta \mathrm{T}$ is restricted by $\mathrm{Eq}(4.4)$

$$
\Delta T \leq L_{e} / C_{b}
$$

Eq. (4.4)

where $C_{b}=5000(\mathrm{~m} / \mathrm{s})$ is assumed as bar wave velocity for steel material. The time step has to be shorter than the time required for the wave to pass by one element. Time step is also relative to the resolution of the FEM output. In FEM simulation, the inverse of time step could be considered as the sampling rate in real experiments. For example, $8 \mathrm{MHz}$ sampling rate could be considered as $0.125 \mu$ sec for time step in FEM simulation.

\subsection{COMPARING ALGOR FEM OUTPUT WITH BOOK RESULT}

In order to show that the FEM can handle wave propagation problems, the experimental results by Miklowitz (1957) are used to compare with the FEM result. A 762 $\mathrm{mm}$ (30 inch) 24S-T aluminum alloy bar is used in his experiment. The diameter of this bar is $25.4 \mathrm{~mm}(1 \mathrm{inch})$. The material properties are: Young's Modules $74.6 \mathrm{Gpa}\left(10.6 * 10^{6}\right.$ psi); Poisson's ratio 0.325; density $2773 \mathrm{Kg} / \mathrm{m} 3 \quad\left(2.590 * 10^{-4} \mathrm{lb}-\mathrm{sec}^{2} / \mathrm{in}^{4}\right)$. The impact loading was closely approximated as a step function. The rise time of this impact is of the order $10^{-8} \mathrm{sec}$, and such a rise time in the $24 \mathrm{~S}-\mathrm{T}$ aluminum alloy rod corresponds to about $5.08 * 10^{-2} \mathrm{~mm}\left(2 * 10^{-3} \mathrm{inch}\right) . \quad$ An axial strain gage was attached on the surface at $508 \mathrm{~mm}$ (20 inch) for measuring the axial-strain. In the FEM simulation, a half Hanning function is made for the FEM input. Figure 4.5 shows the FEM input of the Hanning function. Both 
3-D and Axis-Symmetric FEM models are used to simulate this experiment. The element sizes are set to $0.2032 \mathrm{~mm}$ along the axis and $3.175 \mathrm{~mm}$ along the radius. Figure 4.6 shows the result of Miklowitz's experiment compared with the 3-D FEM model and Axis-symmetric model FEM outputs. In this figure, both the 3-D and Axis-sym model results can match the experimental result well. Having the comparison of these 3 curves in Figure 4.6, a successful FEM simulation is assured. Since the Axis-sym FEM result has better agreement than the 3-D model does, this also implies the advantage of Axis-Sym model.

\subsection{SHORT ROD FEM OUTPUT}

The length of the short rod is $158.75 \mathrm{~mm}$, and the diameter is $4.2 \mathrm{~mm}$. The Young's modulus and the Poisson's ratio are assumed. They are 200GPa and 0.29. The measured density is $7950 \mathrm{~kg} / \mathrm{m}^{3}$. The sine wave input and the pulse input are the same as the real experimental inputs. These input waveforms have been shown in Figures 3.2 to 3.7. There are 100 points in the sine wave inputs and 65 points in the pulse inputs. For the FEM sine wave input, it is not perfectly smooth when only 100 points are plotted, but still accurate enough to describe the input wave. Figures 4.7 to 4.11 show the FEM results from sending in the sine waves at $308 \mathrm{KHz}, 364 \mathrm{KHz}, 400 \mathrm{KHz}, 444 \mathrm{KHz}$, and $500 \mathrm{KHz}$.

\subsection{LONG ROD FEM OUTPUT}

The simulation model length of the long rod is 3 meters, and the diameter is $4.2 \mathrm{~mm}$. The Young's modulus, Poisson's ratio and density are assumed to be 200GPa, 0.29 and

$7950 \mathrm{Kg} / \mathrm{m}^{3}$, respectively. The same input waves are sent into the long rods. Figures 4.12 
to Figure 4.16 show the FEM results for the sine wave inputs. The frequency of these inputs are $308 \mathrm{kHz}, 364 \mathrm{kHz}, 400 \mathrm{kHz}, 444 \mathrm{kHz}$, and $500 \mathrm{kHz}$. Figure 4.17 shows the FEM results from the pulse input.

\subsection{SUMMARY}

This chapter describes the procedure for the FEM analysis by using Algor software. The aluminum bar experiment values are compared with the FEM result in order to assure the FEM works well for wave propagation problems. It is obvious that the FEM model used can handle the ultrasonic wave propagation problems. Therefore, a successful FEM simulation has been conducted. In addition, the Axis-Symmetric model is better than the 3-D model in the rod model. By the short rod and long rod simulation, we are bringing the FEM into a useful level in wave propagation problems. Especially in the long rod simulations, the outputs can nicely show that the waves can be dispersed by different frequencies. This means the FEM model is able to consider the elastic waveguide theory and the acoustoelastic effect. 


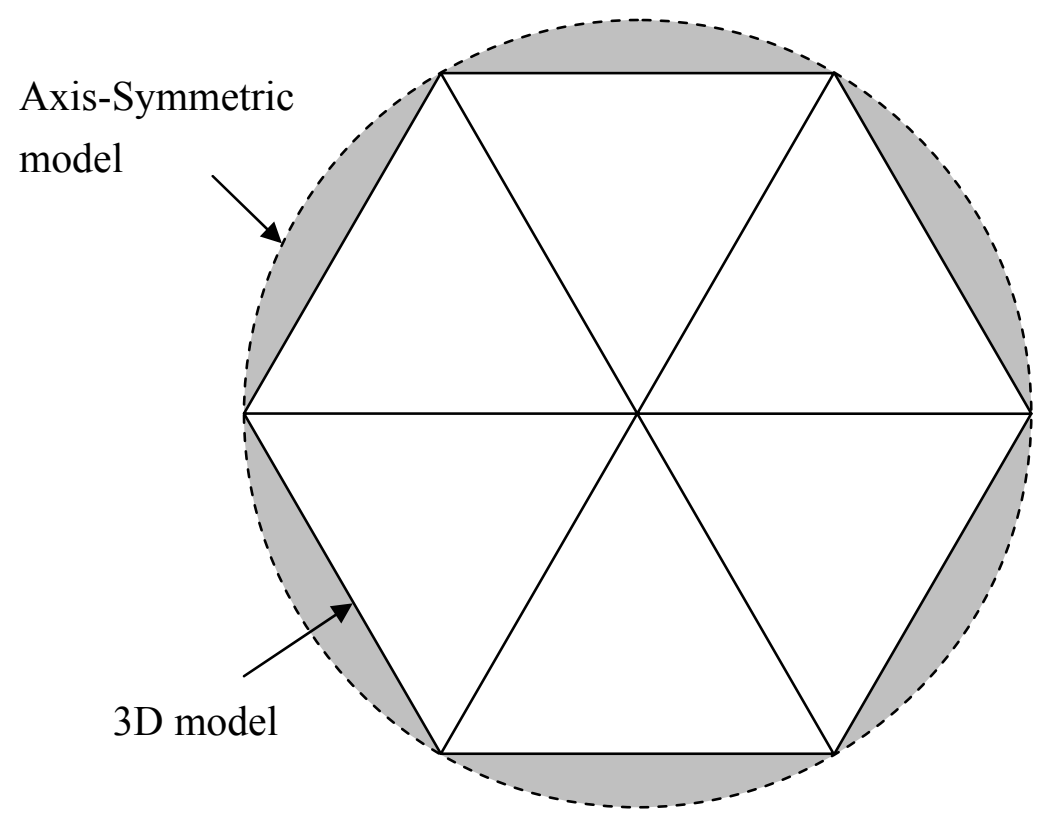

Figure 4.1 The comparison of the cross section between 3-D model and

Axis-Symmetricmodel. Gray area shows the error between these two types of model. 


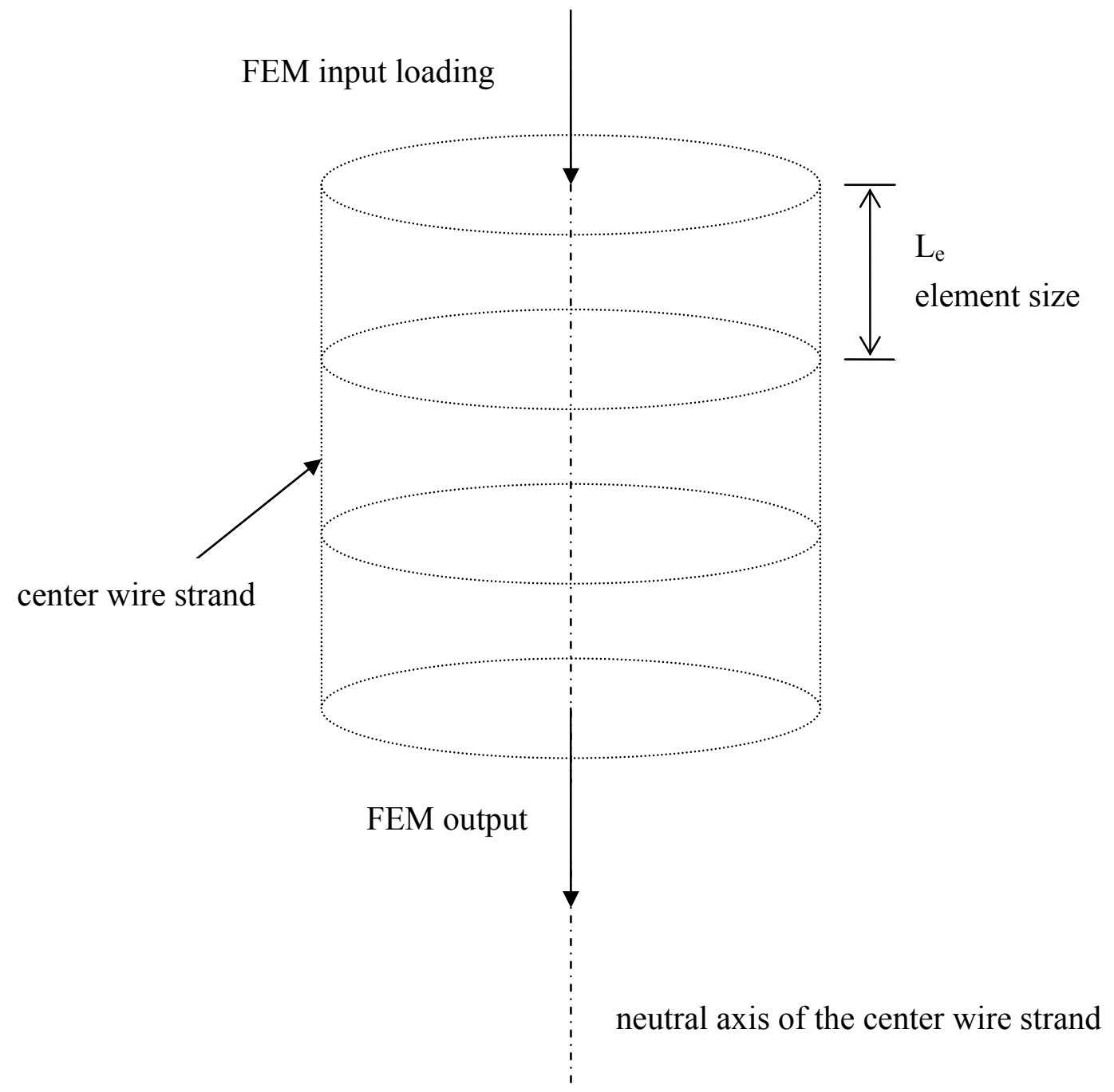

Figure 4.2 The schematic drawing of the FEM simulation 


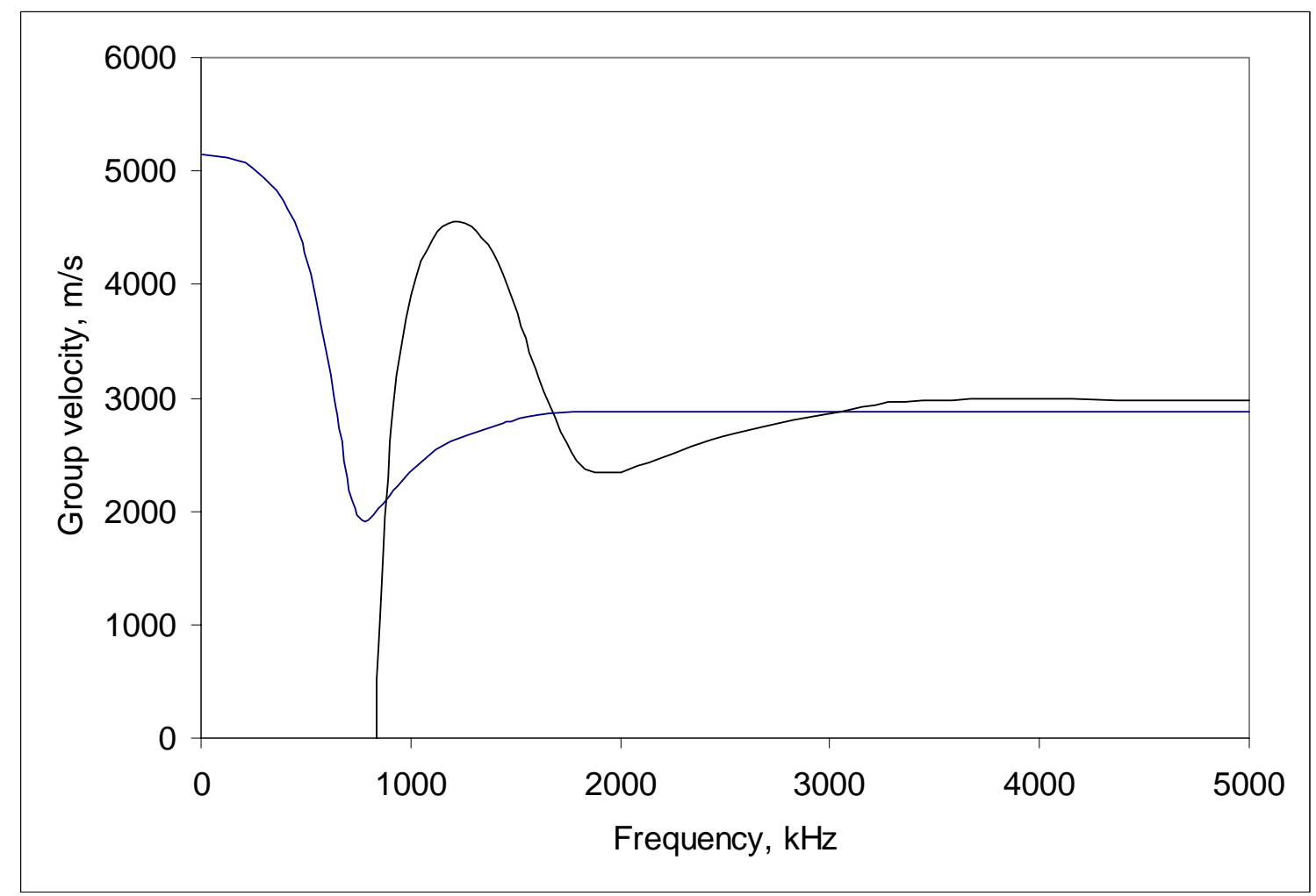

Figure 4.3 Group velocity of the first two longitudinal modes for the center wire $(\varnothing=4.2 \mathrm{~mm}) . \quad$ There is no tensile force applied.

(Chen and Wissawapaisal, 2002) 


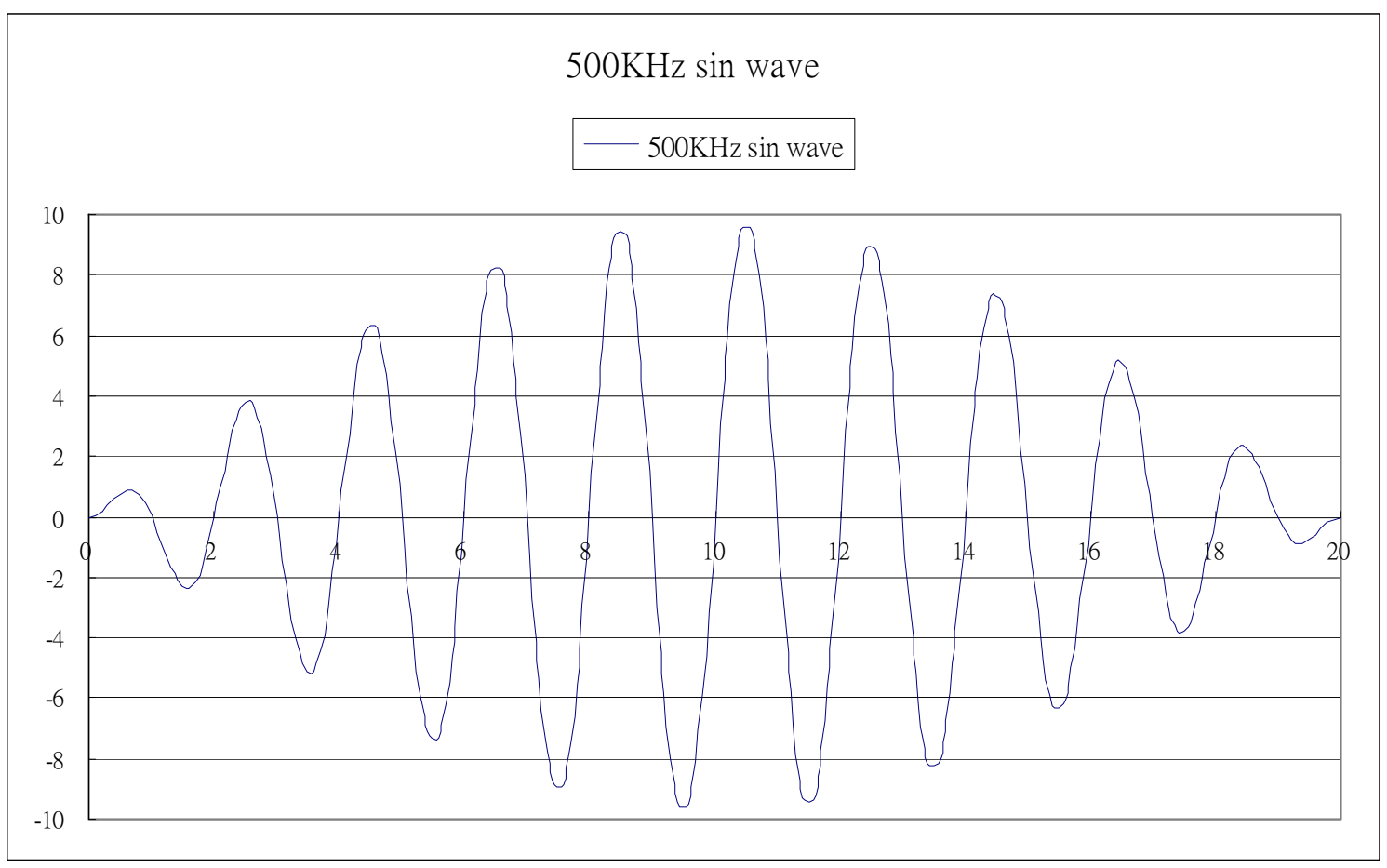

Figure 4.4 The FEM simulation sine wave input at $500 \mathrm{kHz}$

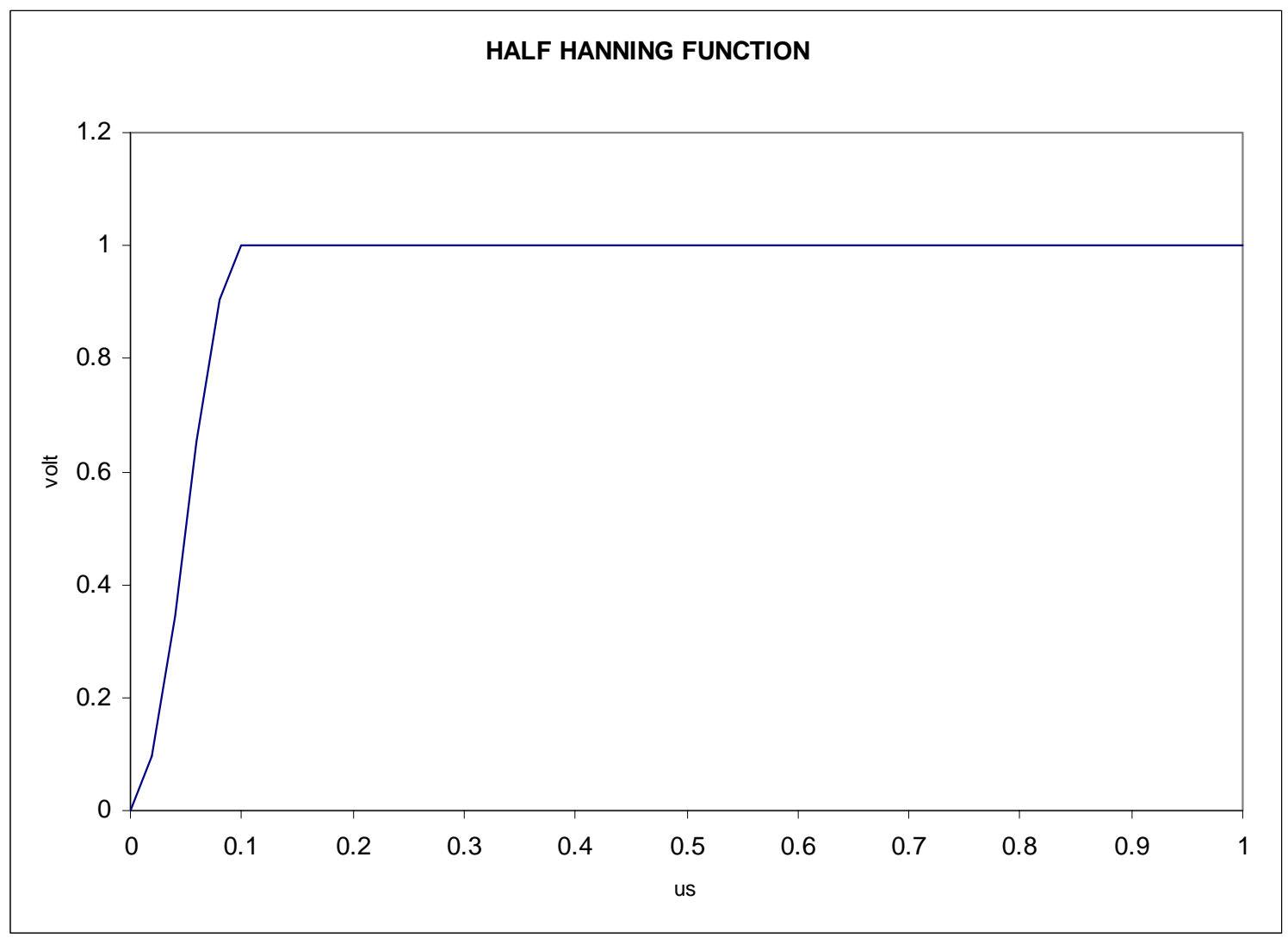

Figure 4.5 The Half Hanning function input 


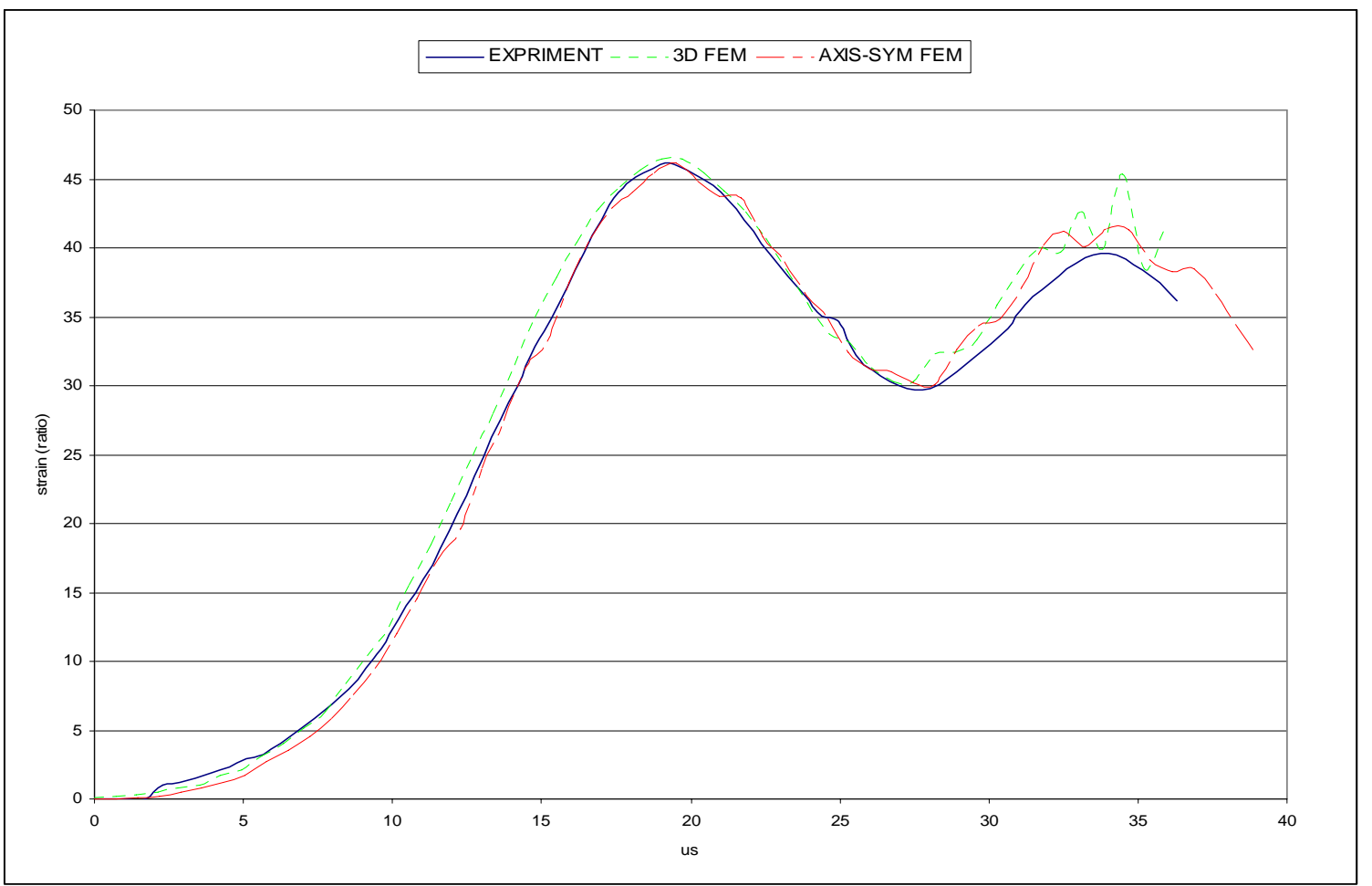

Figure 4.6 Comparison of Miklowitz's experiment result, 3D FEM model result, and Axis-Sym FEM model result

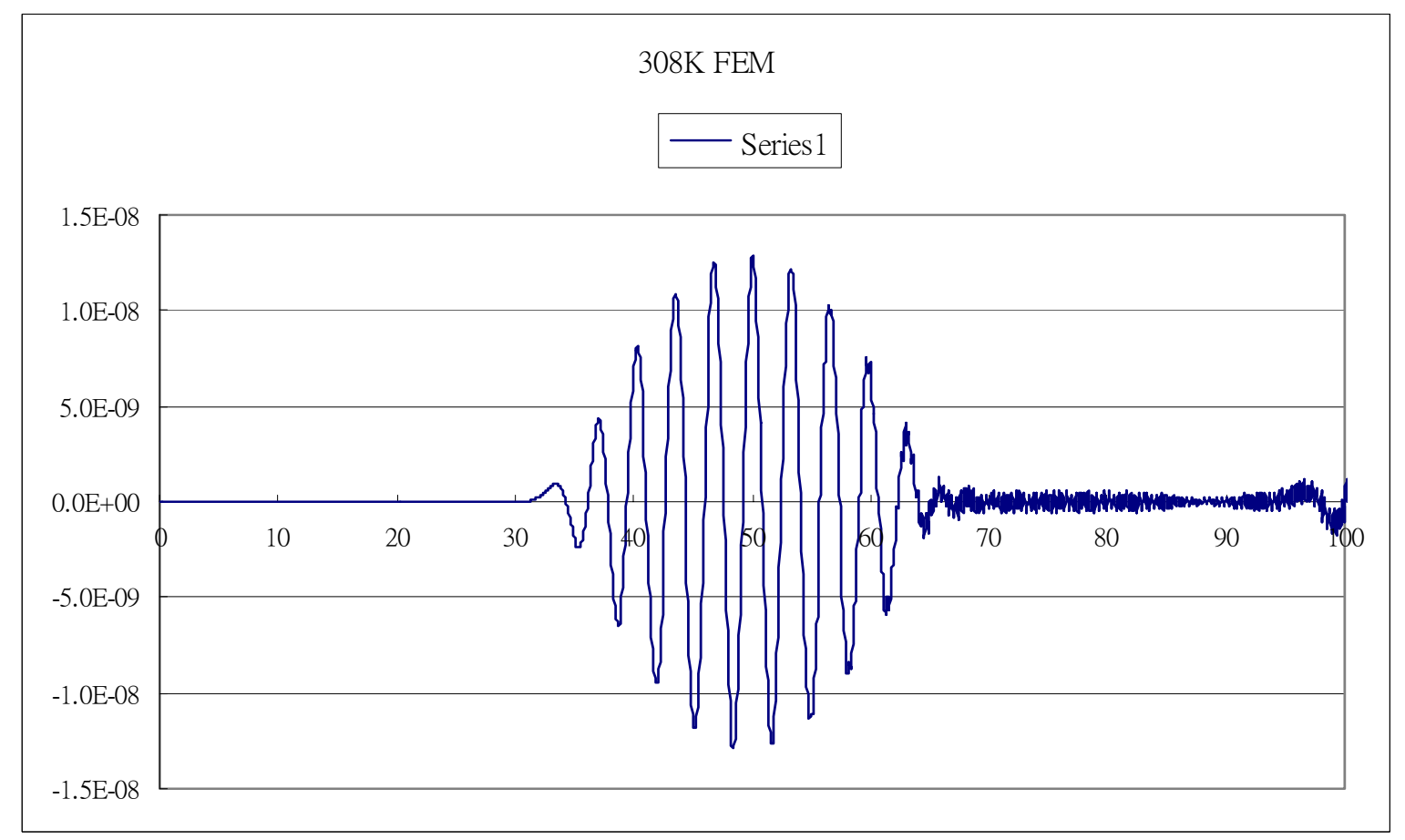

Figure 4.7 The FEM output of the $308 \mathrm{kHz}$ sine wave on the short rod 


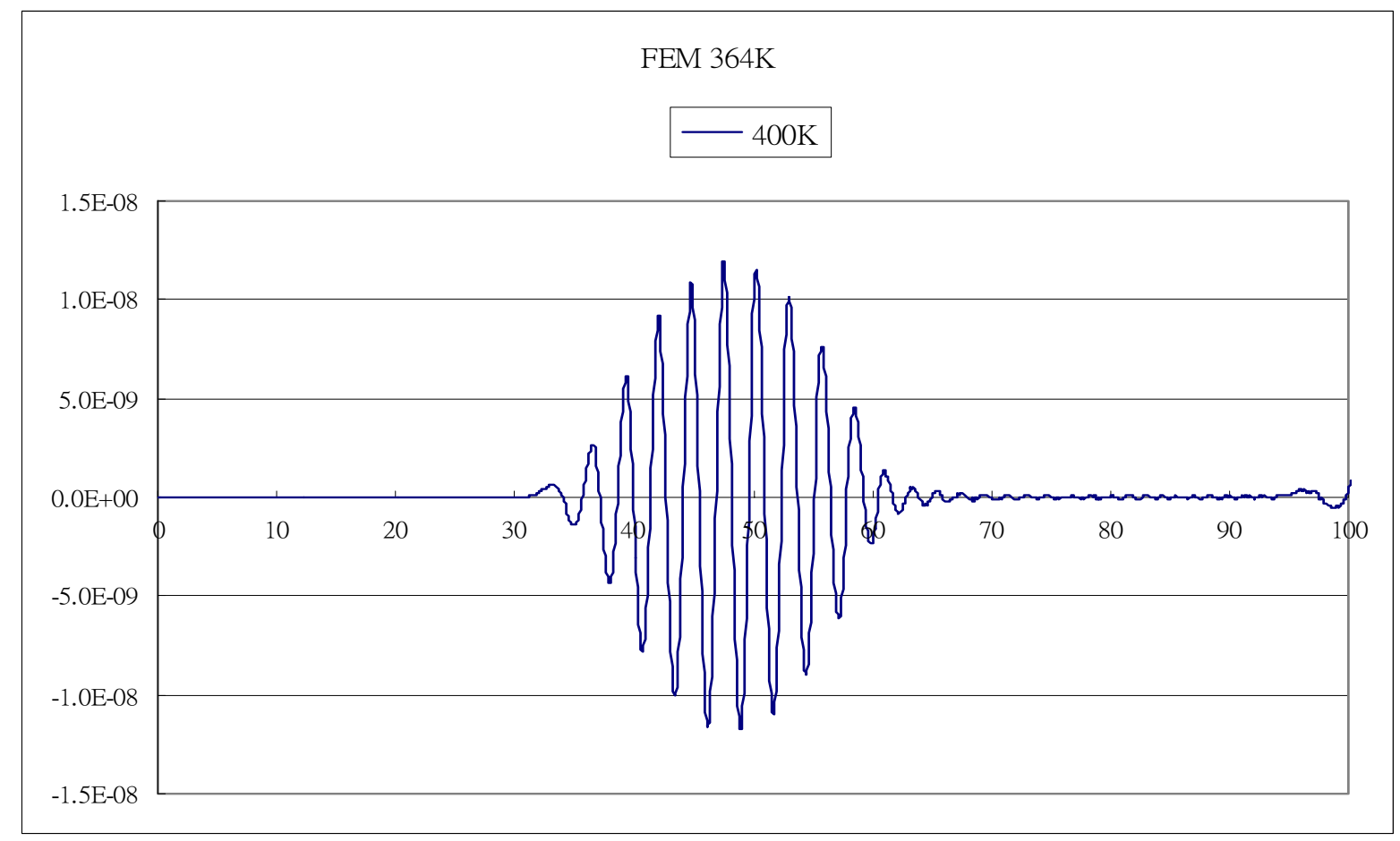

Figure 4.8 The FEM output of the $364 \mathrm{kHz}$ sine wave on the short rod

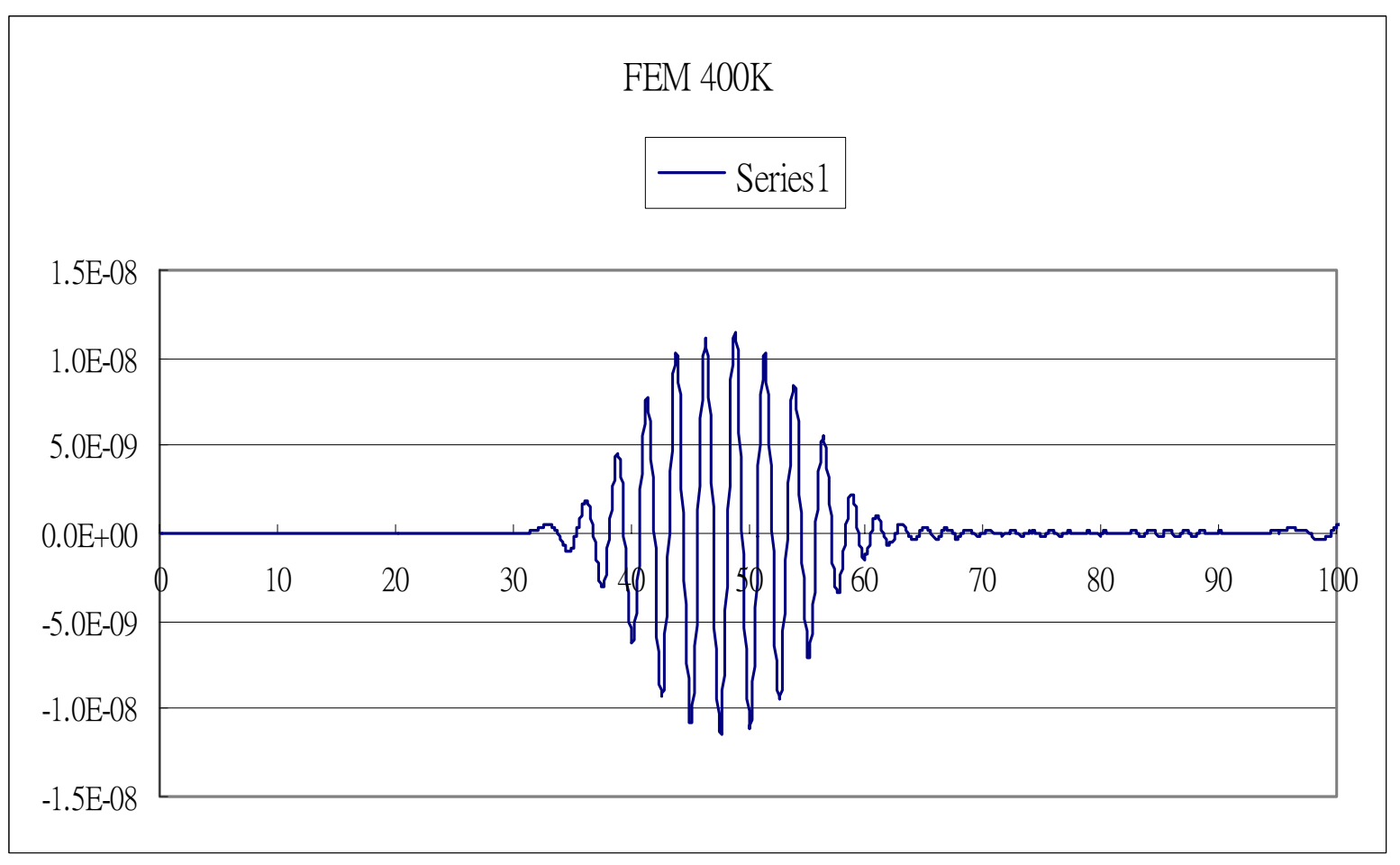

Figure 4.9 The FEM output of the $400 \mathrm{kHz}$ sine wave on the short rod 


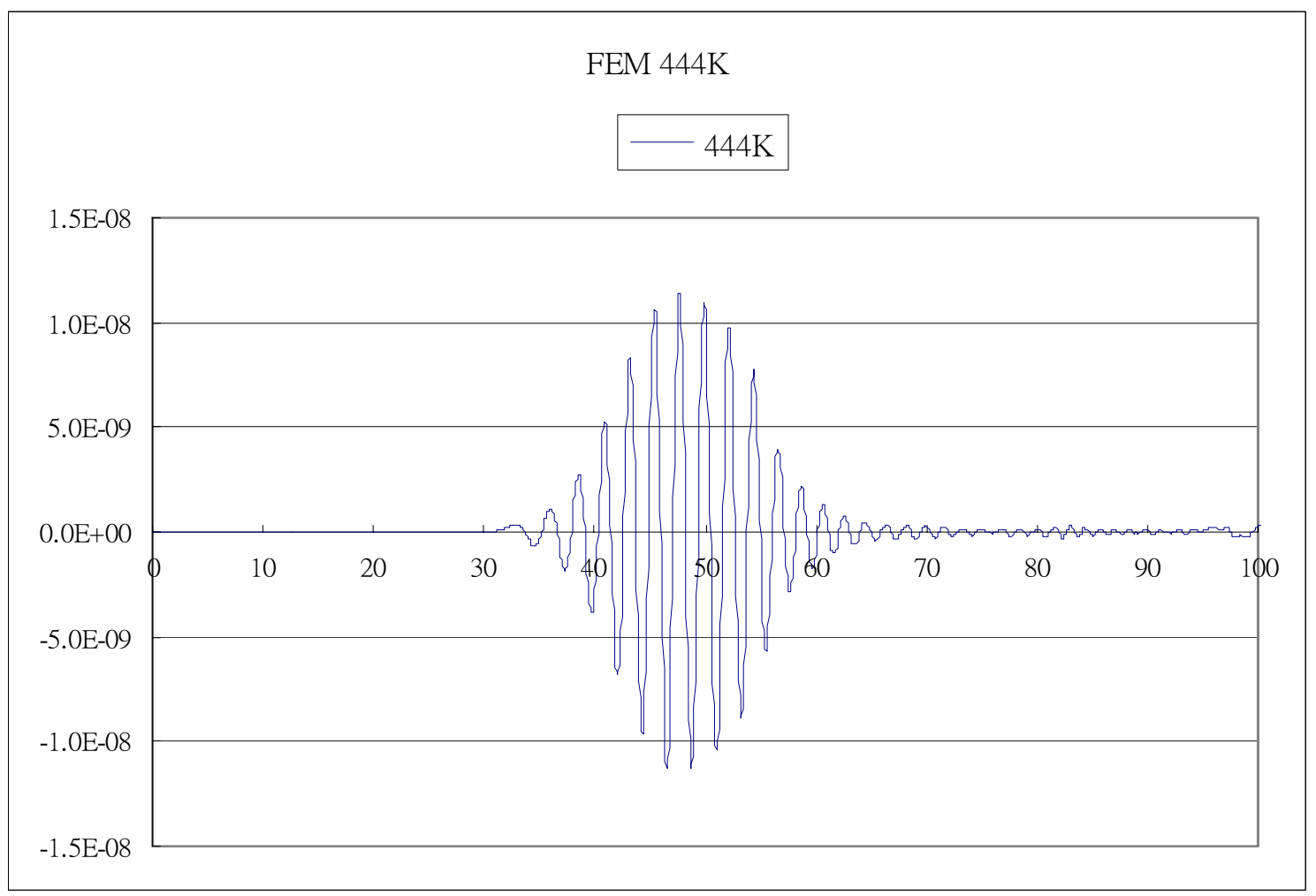

Figure 4.10 The FEM output of the $444 \mathrm{kHz}$ sine wave on the short rod

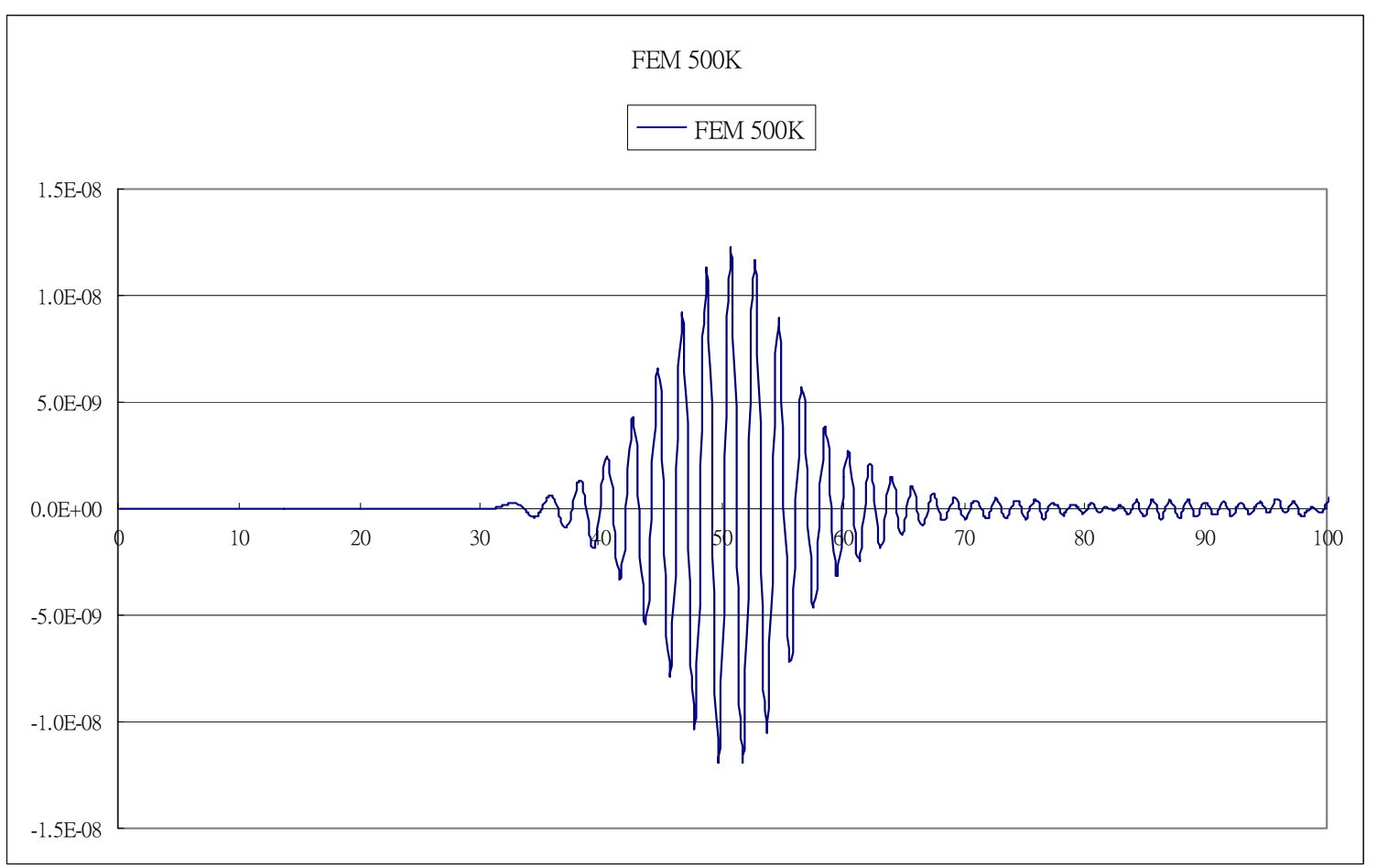

Figure 4.11 The FEM output of the $500 \mathrm{kHz}$ sine wave on the short rod 


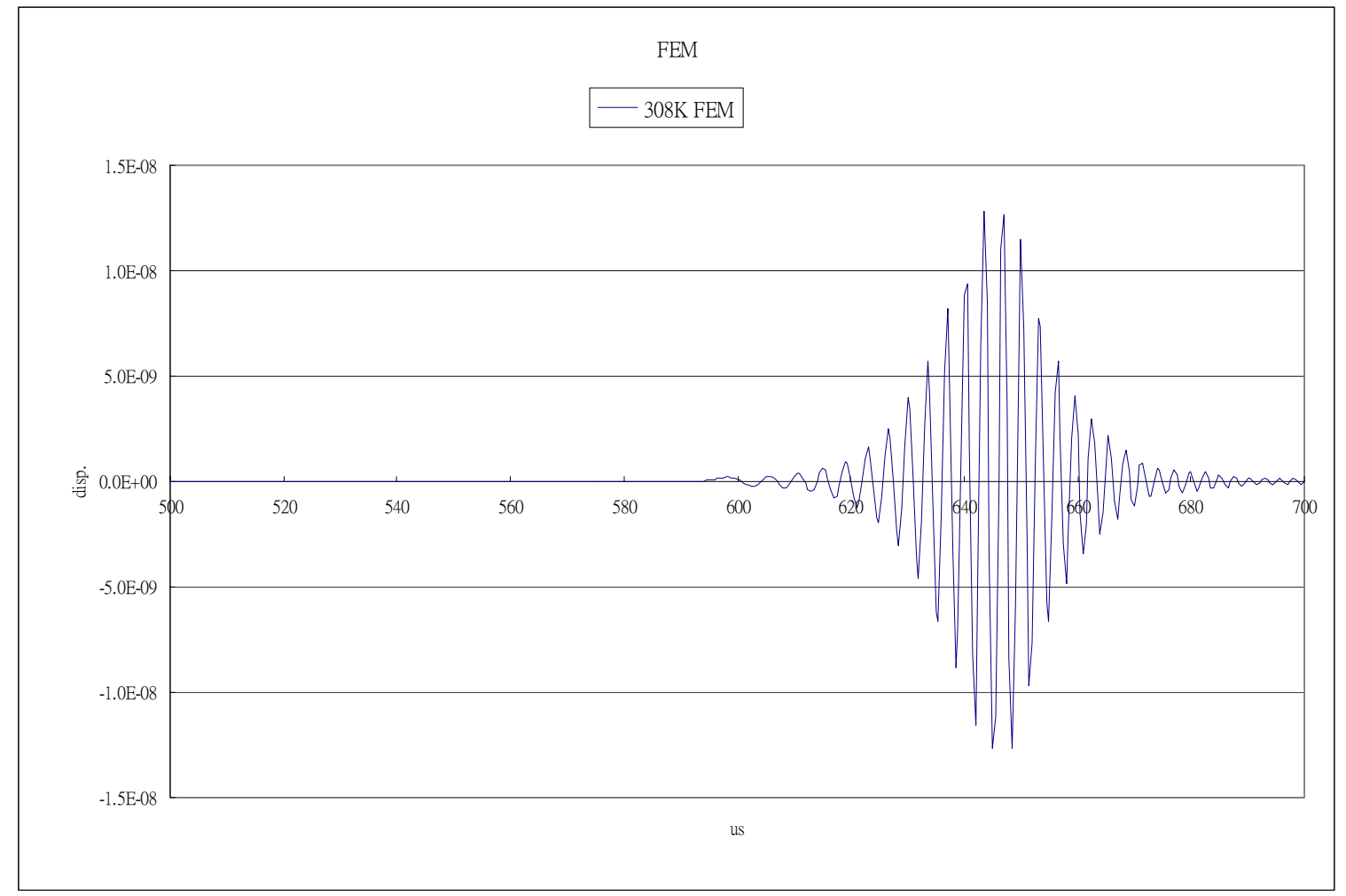

Figure 4.12 The FEM output of the $308 \mathrm{kHz}$ sine wave on the 3 meters rod

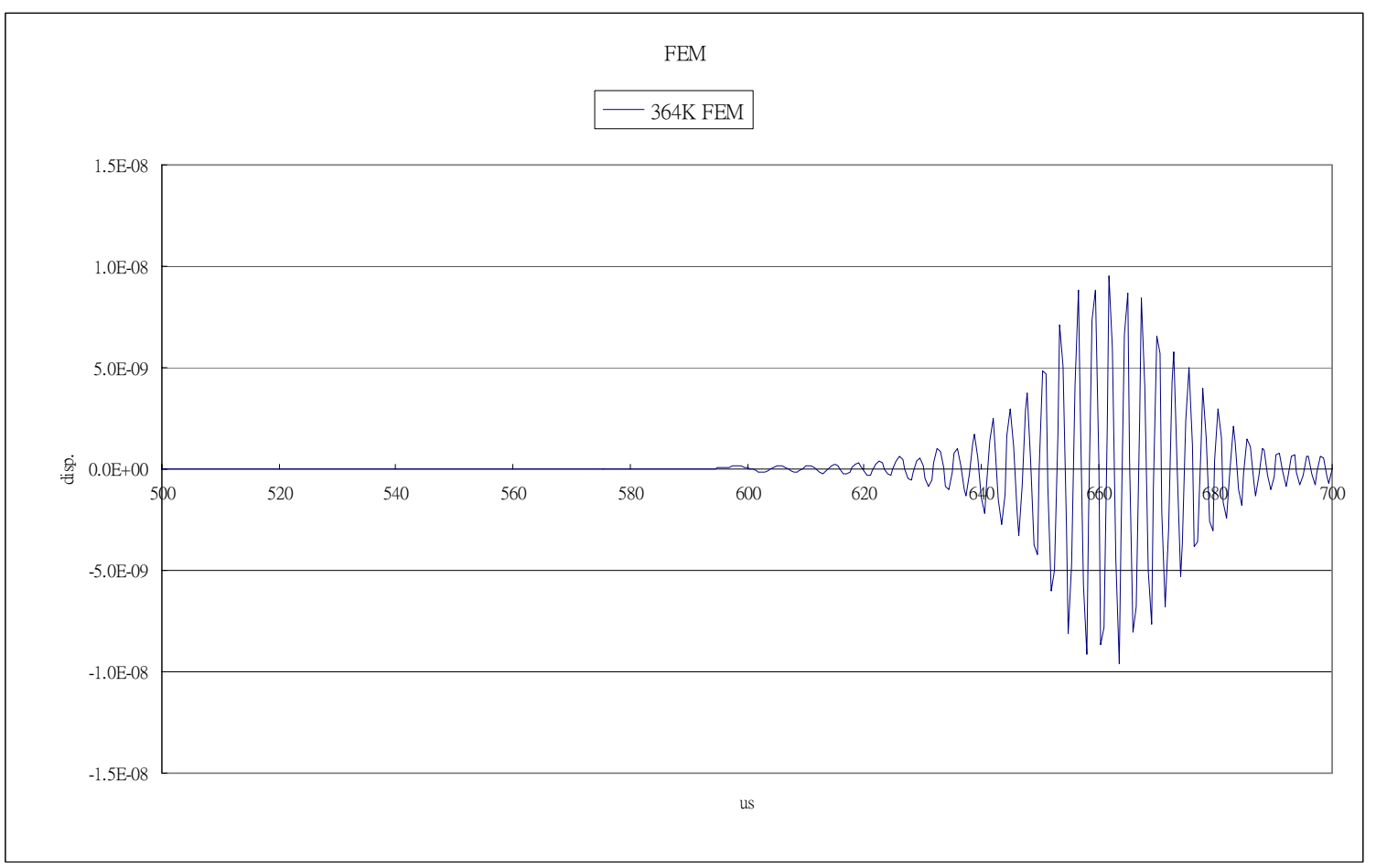

Figure 4.13 The FEM output of the $364 \mathrm{kHz}$ sine wave on the 3 meters rod 


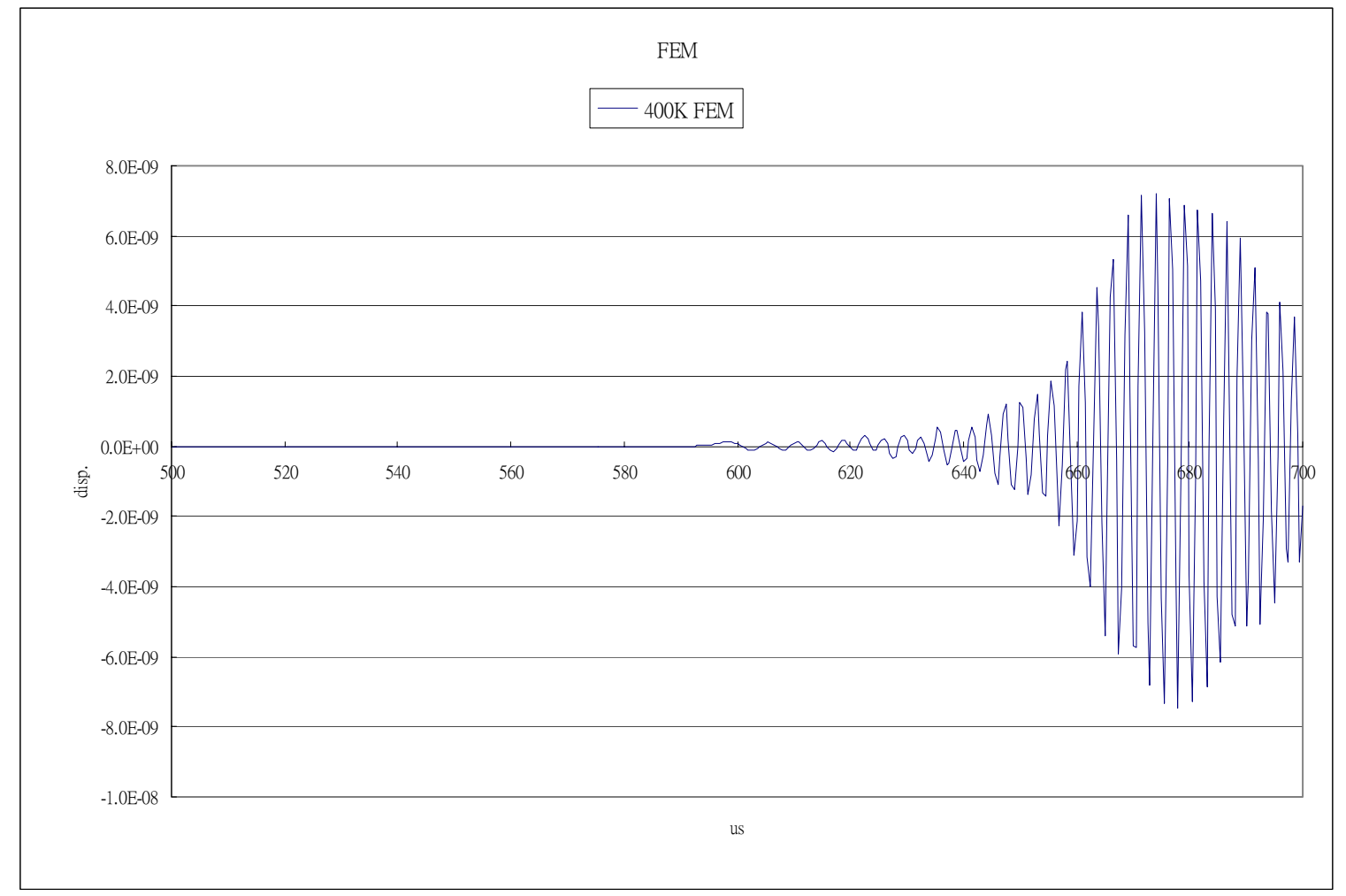

Figure 4.14 The FEM output of the $400 \mathrm{kHz}$ sine wave on the 3 meters rod

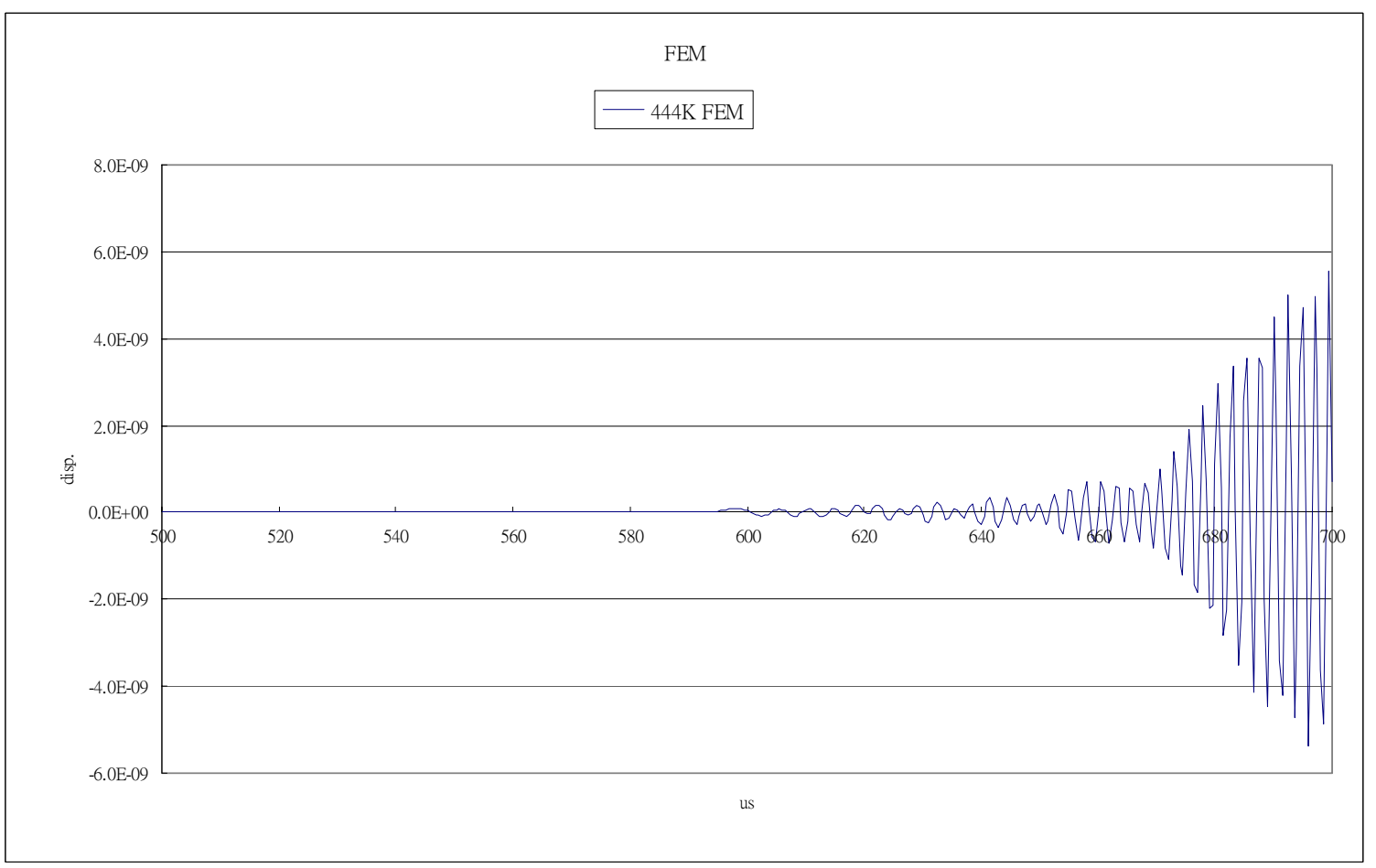

Figure 4.15 The FEM output of the $444 \mathrm{kHz}$ sine wave on the 3 meters rod 


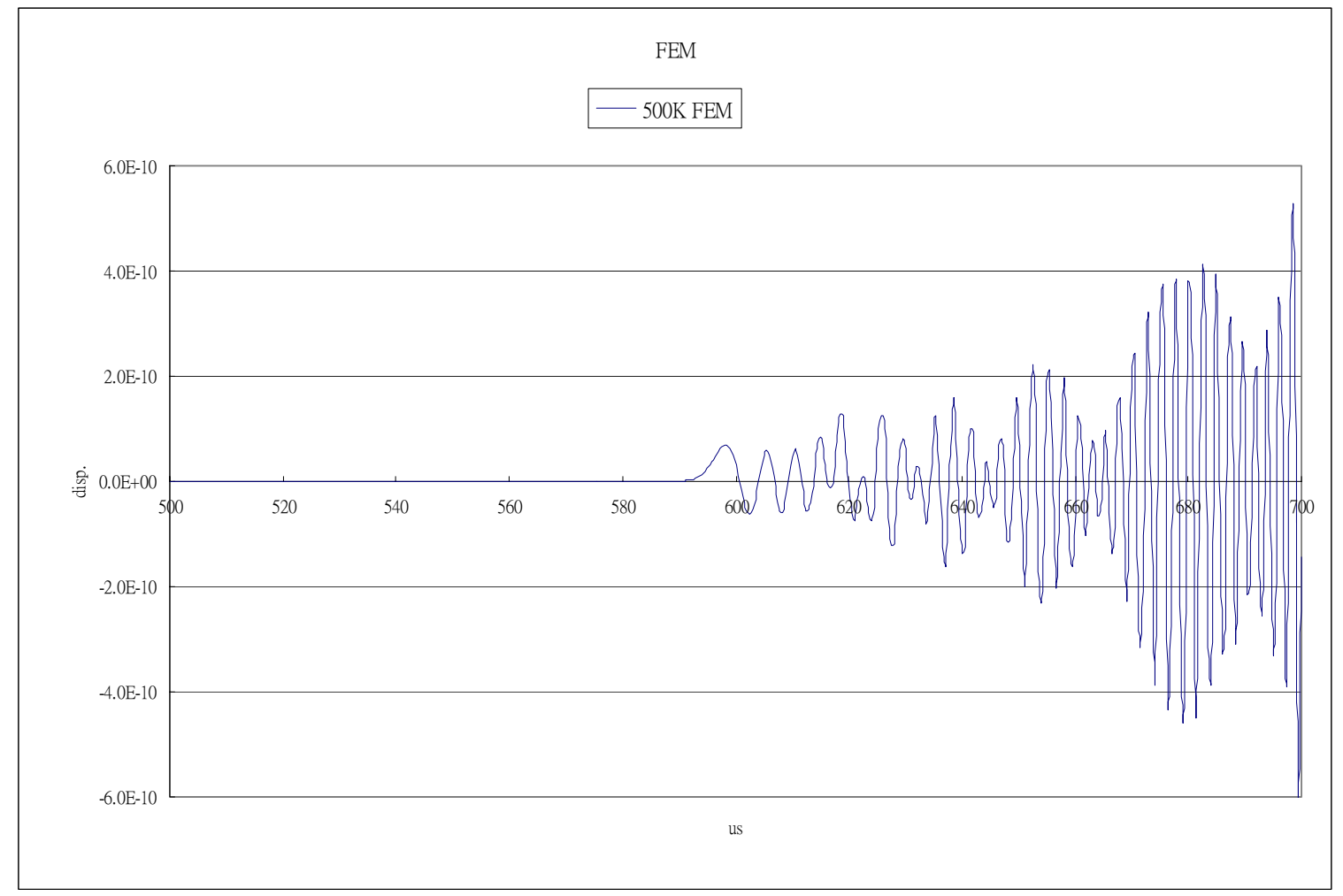

Figure 4.16 The FEM output of the $500 \mathrm{kHz}$ sine wave on 3 the meters rod

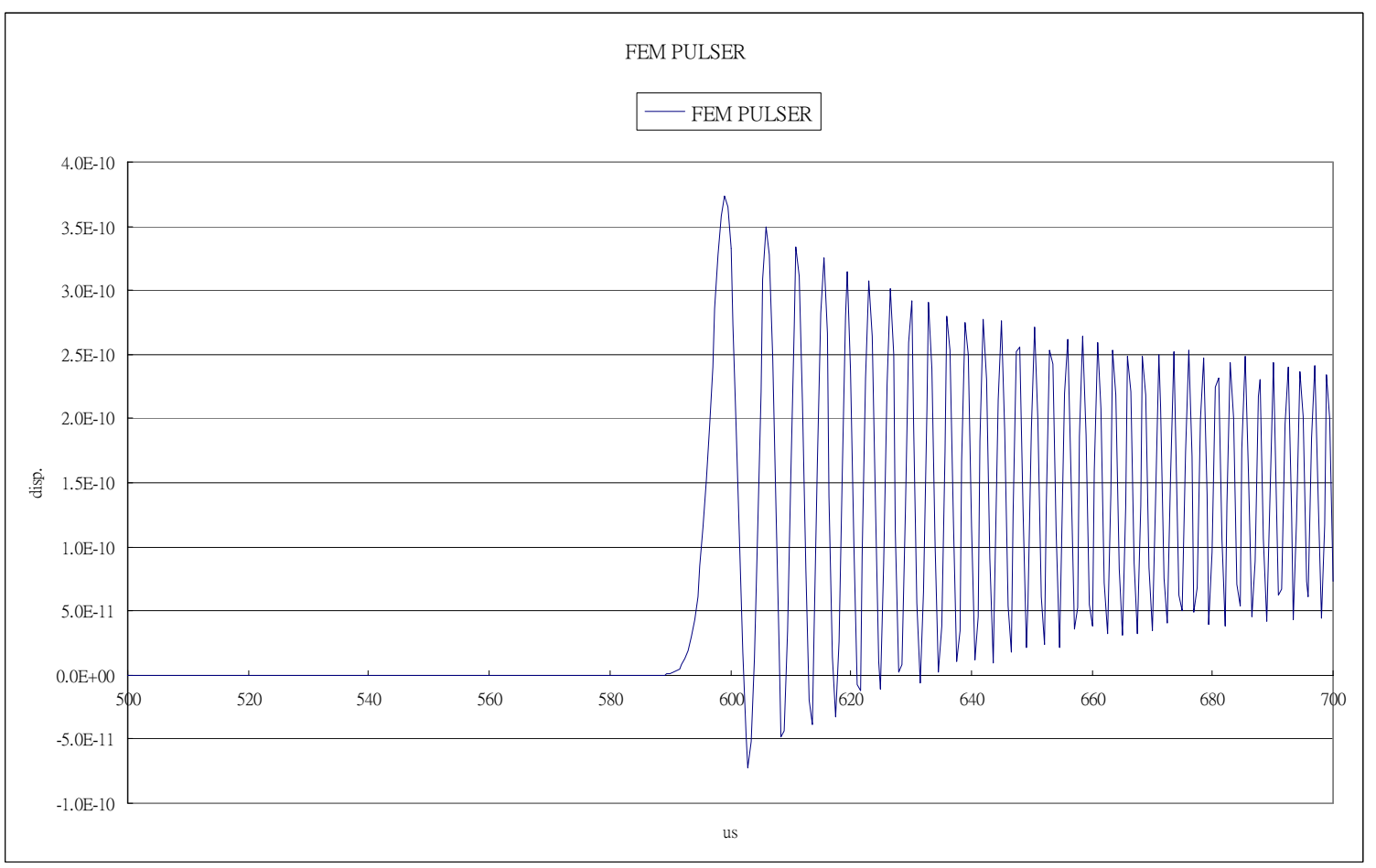

Figure 4.17 The FEM output of the pulse generator input on the 3 meters rod 


\section{CHAPTER 5}

\section{COMPARISON BETWEEN EXPERIMENTAL RESULT AND FINITE ELEMENT METHODS OUTPUT}

\subsection{OVERVIEW}

In this chapter, both the experimental result and the FEM output will be compared in the arrival time, waveform, and Wigner-Ville Transform. The amplitude level of the FEM results has been enlarged in order to compare with the experimental result.

\subsection{SHORT ROD}

\subsubsection{Arrival Time}

Figure 5.1 to 5.5 show the experimental and FEM output waveform on sine wave input. Figure 5.6 shows the output comparison of the pulse generator input. In the experimental results, the arrival time is found to be $31 \mu \mathrm{sec}$. In the FEM results from sine wave input (Figure 5.1 to 5.5), the arrival time is about the same value. The experimental and FEM bar velocity is $5121 \mathrm{~m} / \mathrm{s}$. The theoretical bar velocity is $5015 \mathrm{~m} / \mathrm{s}$ for $\mathrm{E}=200 \mathrm{GPa}$ and $\rho=7950 \mathrm{~kg} / \mathrm{m}^{3}$.

Figure 5.6 shows the output from a wide frequency input waveform with much higher level of trigger. Therefore, the first arrival cycle can be clearly recognized as the low frequency component. In addition, both experimental and FEM results arrive at the same 
time of about $30 \mu \mathrm{sec}$. The bar velocity of experimental and FEM result is seen as $5292 \mathrm{~m} / \mathrm{s}$ from the first arrival.

\subsubsection{Waveform}

In the short rod comparison figures (Figure 5.1 to 5.5), both the waveforms of experiment and FEM showed little distortion, long after propagating with those narrow frequency inputs. Therefore, the Figure 5.1 to 5.5 can still remain the similar waveform as the input. Figure 5.6 is the result generated by pulse input, which contains a wide frequency range. The dispersion effect is clearly shown even though the pulse only propagates through a $15.9 \mathrm{~cm}$ short rod.

\subsubsection{Wigner-Ville Transform (WVT)}

Figure 5.7 to 5.18 present the Wigner-Ville Transform applied on short rod outputs. Figure 5.7 to 5.16 present the WVT applied on the outputs of sine wave inputs. Once again, the time-frequency domain figures couldn't show the evident effect of dispersion on different frequencies because of the narrow band input frequencies and insufficient rod length. Both of the experimental and FEM transformations show no clear slope in the time-frequency plot. Figure 5.17 and 5.18 shows the transformation of the output from pulse input. Figure 5.17 shows a line with slope on higher frequencies, the lower frequencies didn't disperse very well due to the length of the rod. 


\subsection{LONG ROD}

\subsubsection{Arrival time}

Figure 5.19 to 5.23 show the waveform comparison between experimental and FEM result for the long rod. In order to see a much clear arrival time, figure 5.24 to 5.28 show the zoom-in waveform of the experimental output from. Having the sine wave inputs, the experimental arrival time is around $575 \mu \mathrm{sec}$ and the FEM arrival time is about $590 \mu \mathrm{sec}$. From the experimental results, the bar velocity is $5217 \mathrm{~m} / \mathrm{s}$ and from the FEM result, it is $5085 \mathrm{~m} / \mathrm{s}$. The theoretical bar velocity is $5031 \mathrm{~m} / \mathrm{s}$ for $\mathrm{E}=200 \mathrm{GPa}$ and $\rho=7900 \mathrm{~kg} / \mathrm{m}^{3}$. The difference between the FEM and experimental results is due to the assumed material properties in the calculation $\left(\mathrm{E}=200 \mathrm{GP}_{\mathrm{a}}, \nu=0.29, \rho=7950 \mathrm{~kg} / \mathrm{m}^{3}\right)$.

Figure 5.29 presents the output comparison from C101HV pulse generator. The experimental arrival time is $578 \mu \mathrm{sec}$ and the FEM arrival time is $590 \mu \mathrm{sec}$.

\subsubsection{Waveform}

The experimental and FEM results show that the output waveforms are similar to each other under the sine input. Even though the peaks occur at different times, they still possess the same shape. In addition, the cycle numbers are increased in both experimental and FEM results. Figure 5.29 shows more difference in the comparison, there is a constant offset in the FEM output. The offset means that there is a permanent displacement, which is not possible in the reality. Due to the contacting of the specimen at supports, the permanent displacement is not presented in the experimental result. In the FEM simulations, the 
models are set to be with free boundary conditions. Having this, the model can move freely without any restriction and therefore, the offset will occur if the loading is not exactly positive and negative balanced.

\subsubsection{Wigner-Ville Transform}

The signal points between $500 \mu \mathrm{sec}$ and $700 \mu \mathrm{sec}$ are collected for the application of WVT. This means $500 \mu \mathrm{sec}$ should be added to the time domain when reading the figures. Figure 5.30 to 5.39 show the WVT applied on the results due to sine wave input. Figure 5.40 and 5.41 show the WVT on the pulse generator output. In the 3 meter rod cases, it is easy to see the frequency dispersion effect on experimental and FEM results. In the $500 \mathrm{kHz}$ frequency case (Figure 5.39 and 5.40), the output frequency component is shown to be lower then $500 \mathrm{kHz}$. This is because the $500 \mathrm{kHz}$ wave velocity is slower and falls behind 700 $\mu$ sec. Both of experimental and FEM WVT figures show the same behavior.

Figure 5.41 and 5.42 show the WVT applied on outputs due to the C101HV pulse generator inputs. There is a constant offset in the FEM result which means that there is

permanent displacement. Having the offset will cause a low frequency with very high amplitude in the spectrum diagram. Even though there is a offset in Figure 5.42, a curve of frequency dispersion effect still can be formed. This curve compares with the curve in Figure 5.41. 


\subsection{SUMMARY}

In this chapter, both experimental and FEM simulation result were compared in time-amplitude and time-frequency domain (applied by WVT). The FEM numerical results correlated well with the experimental result. However, due to the assumption of material properties, the arrival time and the waveform couldn't be exactly the same. In addition, the wave reflections in the sensors also affect the experimental waveform. The FEM outputs, due to the sine wave inputs, have better comparison than that of the experimental outputs due to the pulse input. This is because we can control our input signal by the DS345 function generator, but not for the C101HV pulse generator. Even the pulse generator can trigger the PICO sensor by the same negative pulse function, but the exact waveform responded by PICO sensor can not be determined. Having this, the waveforms sent into the FEM model and the waveform sent into the specimen by PICO sensor can not be identical, which caused more differences between the experimental and the FEM output. 


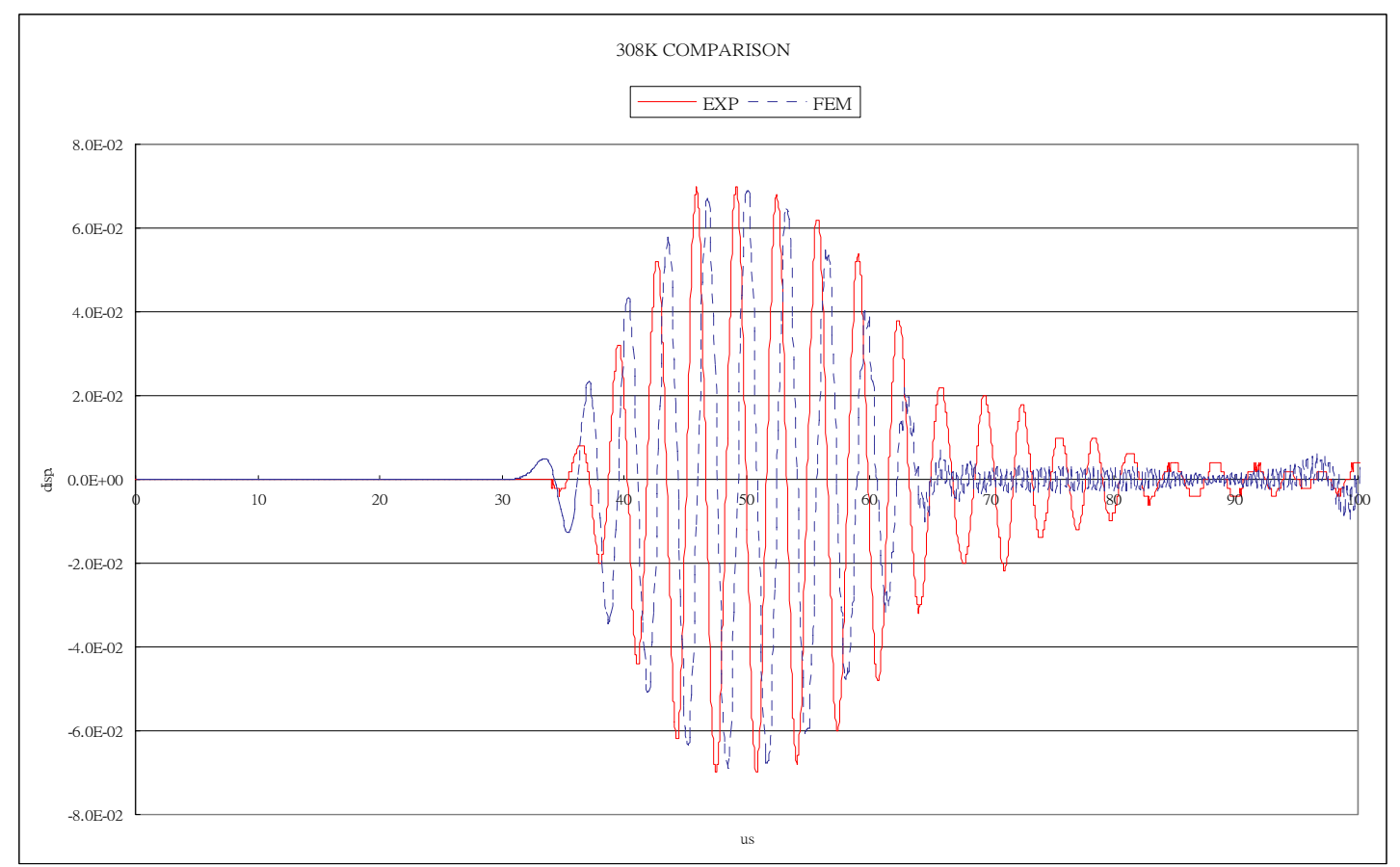

Figure 5.1 The waveform comparison between the experimental and FEM result from $308 \mathrm{kHz}$ sine wave input.

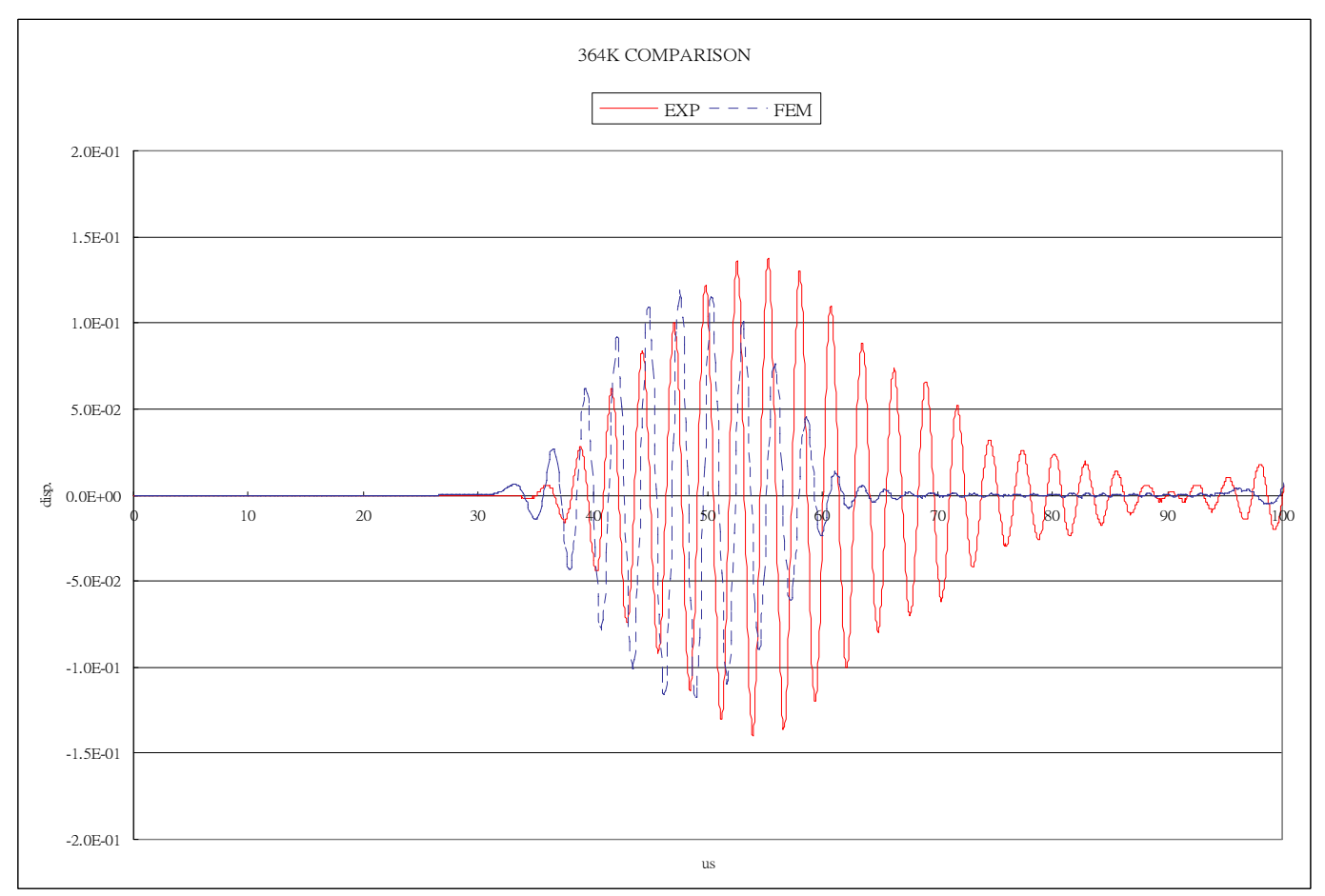

Figure 5.2 The waveform comparison between the experimental and FEM result from

$364 \mathrm{kHz}$ sine wave input. 


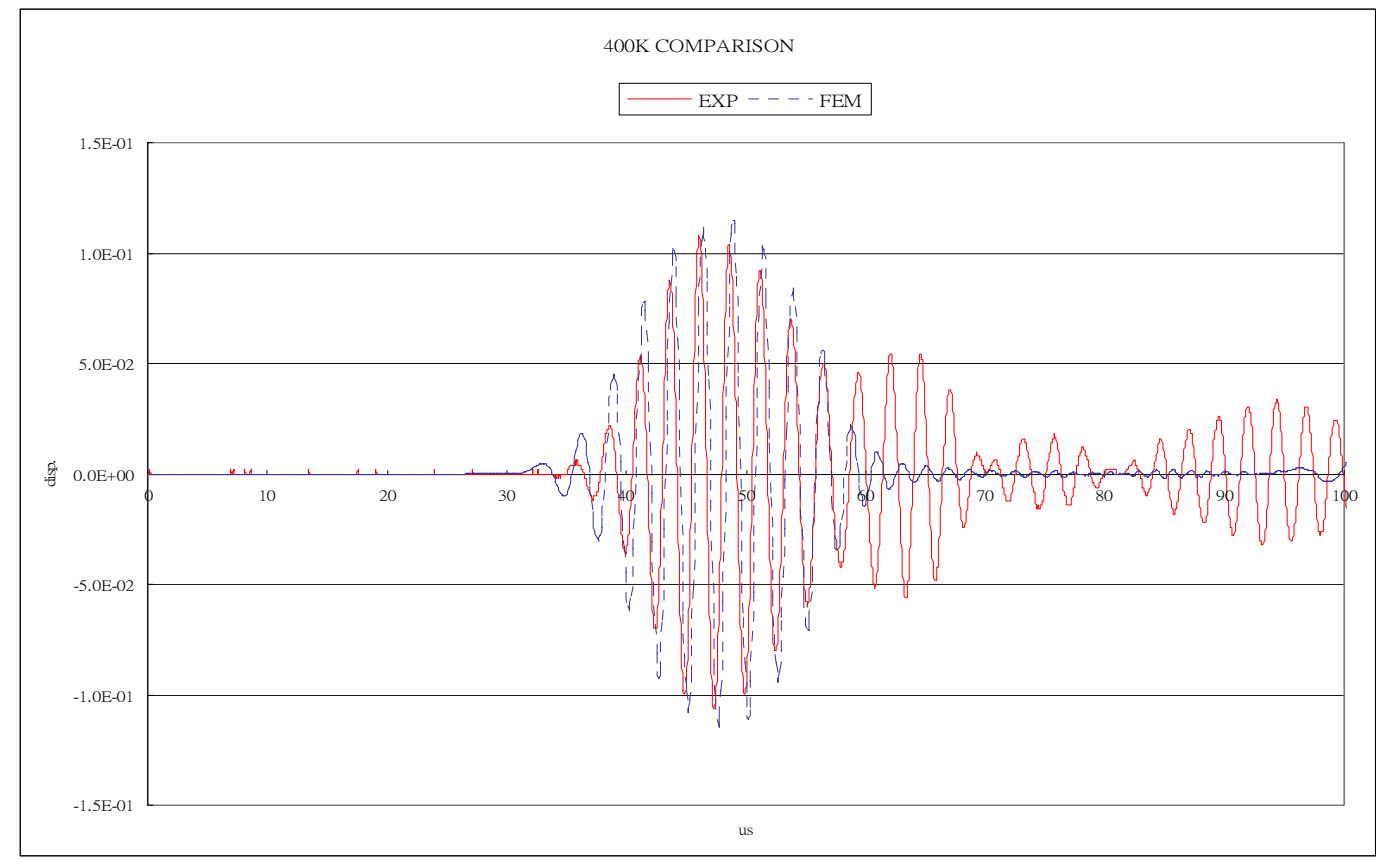

Figure 5.3 The waveform comparison between the experimental and FEM result from $400 \mathrm{kHz}$ sine wave input.

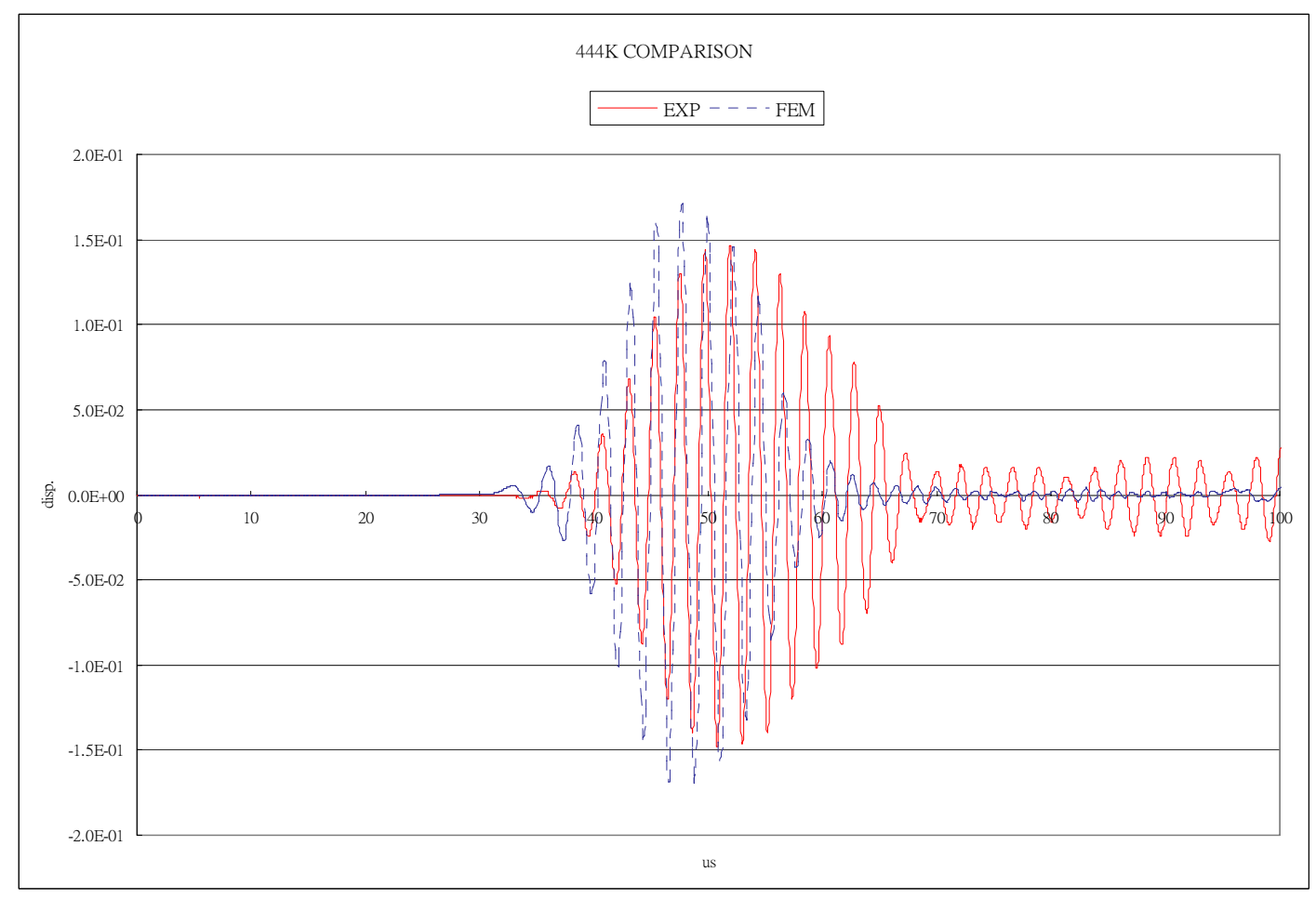

Figure 5.4 The waveform comparison between the experimental and FEM result from $444 \mathrm{kHz}$ sine wave input. 


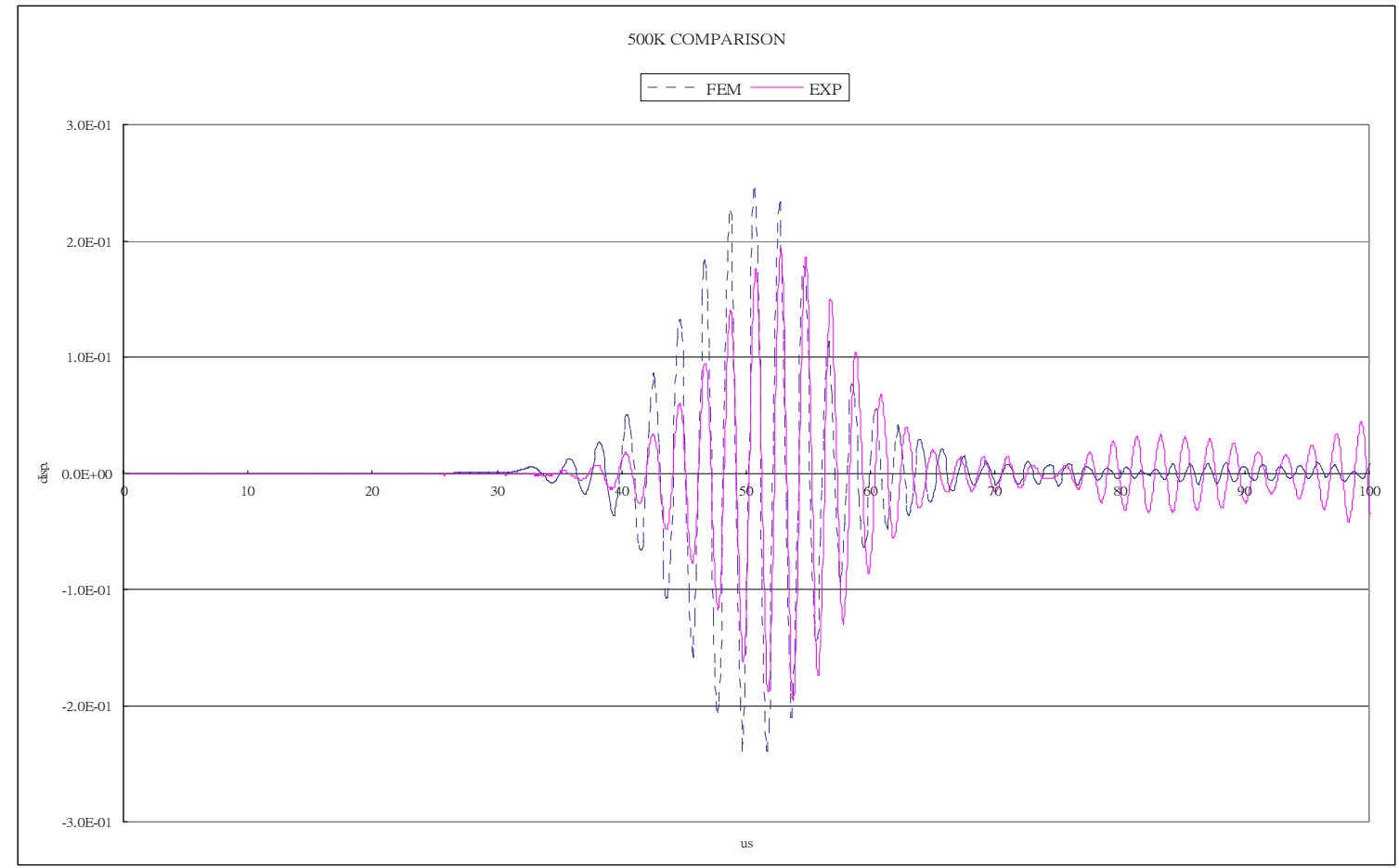

Figure 5.5 The waveform comparison between the experimental and FEM result from $500 \mathrm{kHz}$ sine wave input.

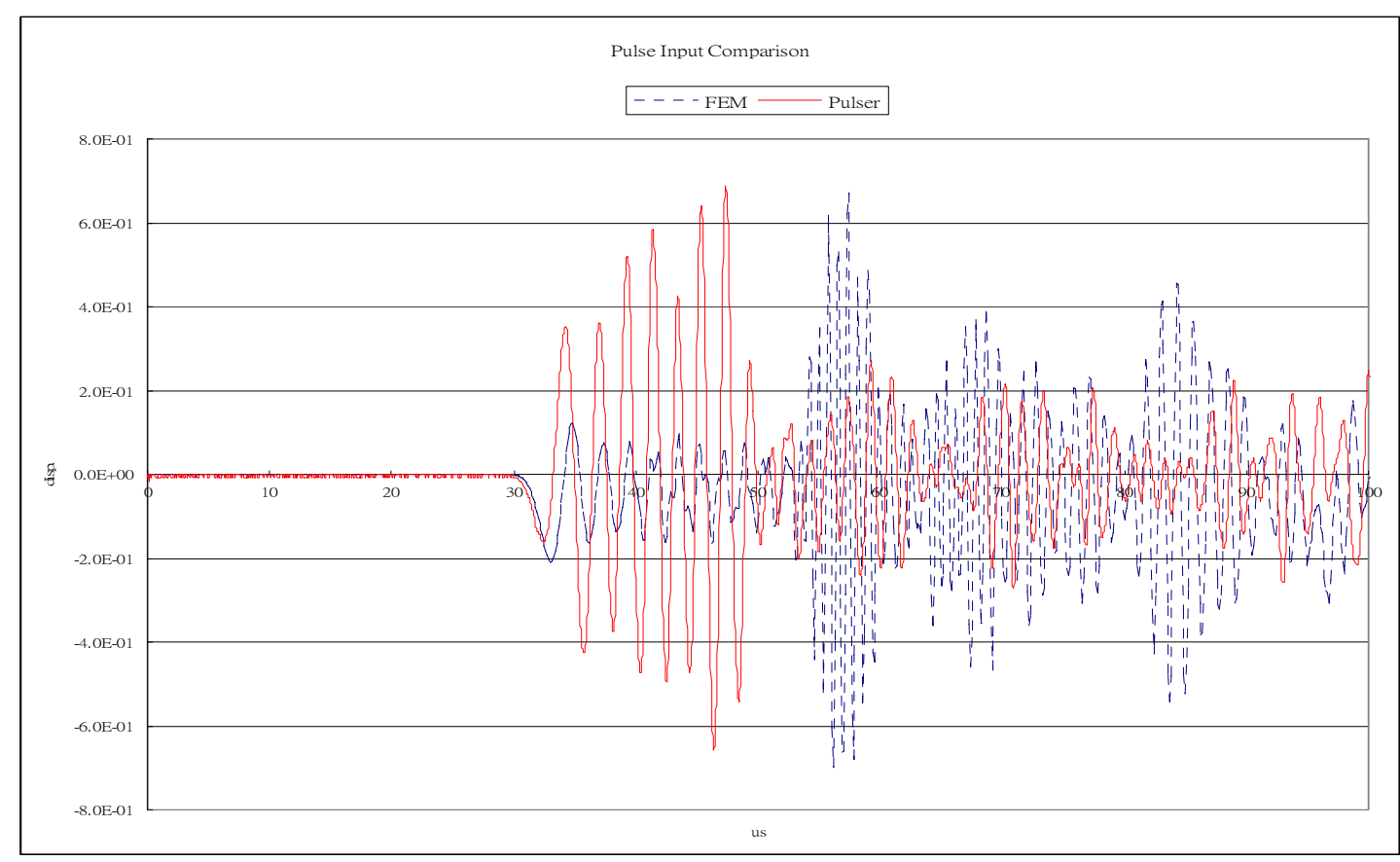

Figure 5.6 The waveform comparison between the experimental and FEM result from the C101HV pulse generator input. 

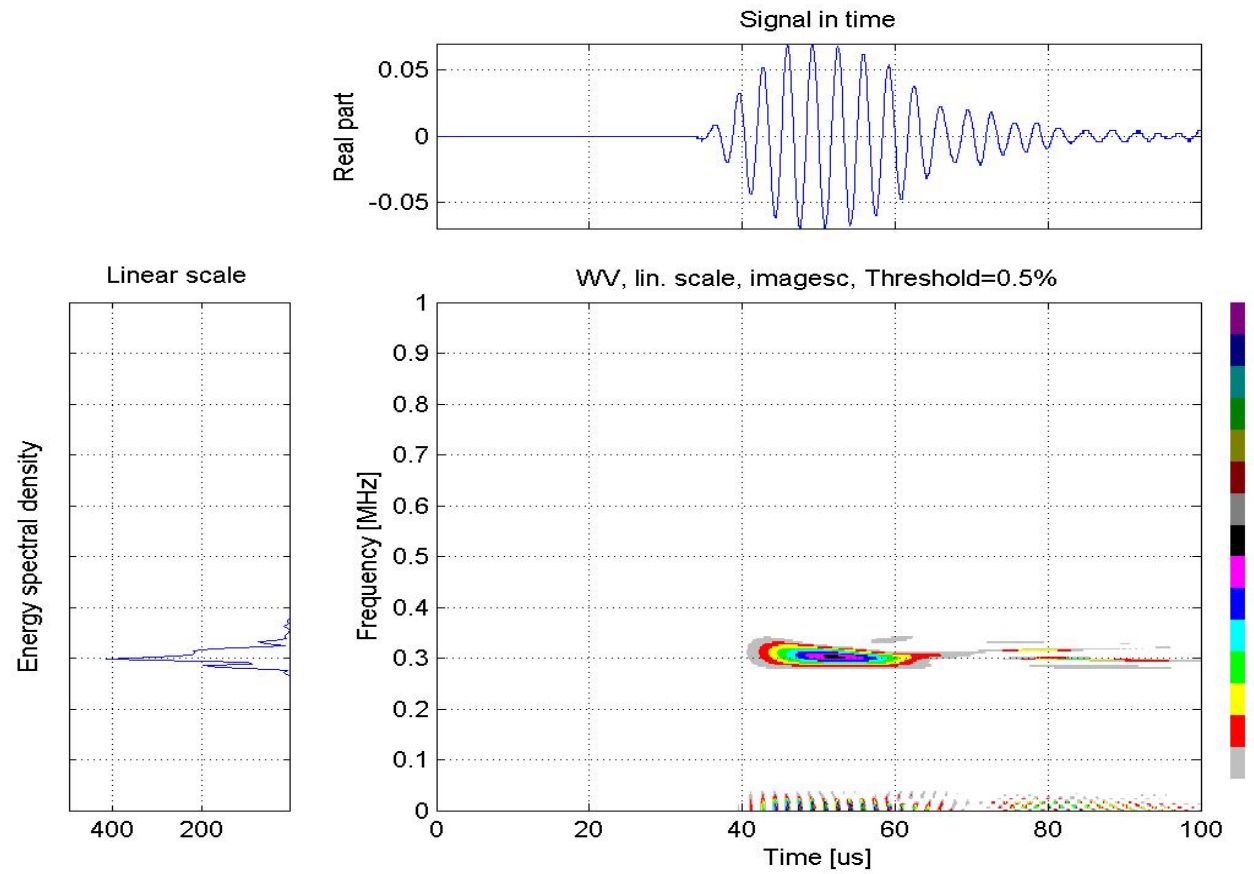

Figure 5.7 The Wigner-Ville Transform of $308 \mathrm{kHz}$ sine wave experiment output on the short $\operatorname{rod}$
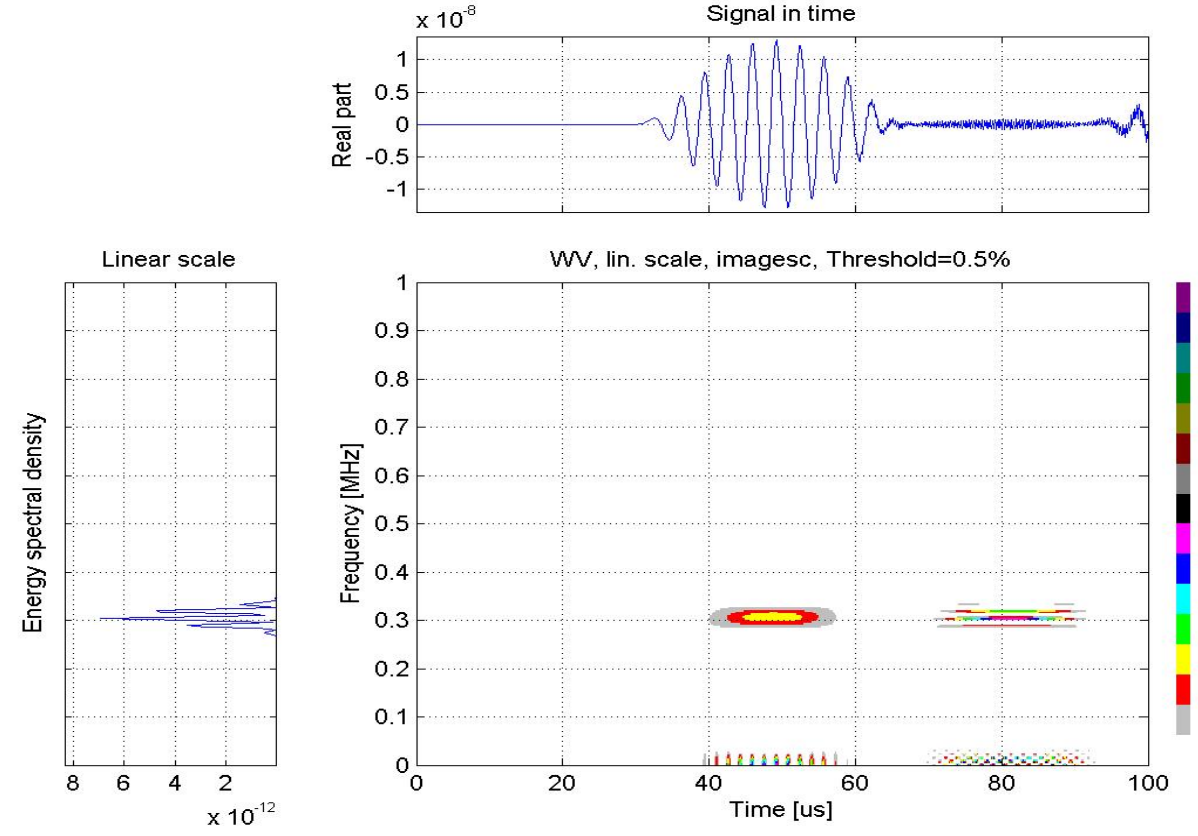

Figure 5.8 The Wigner-Ville Transform of $308 \mathrm{kHz}$ sine wave FEM output on the short rod 

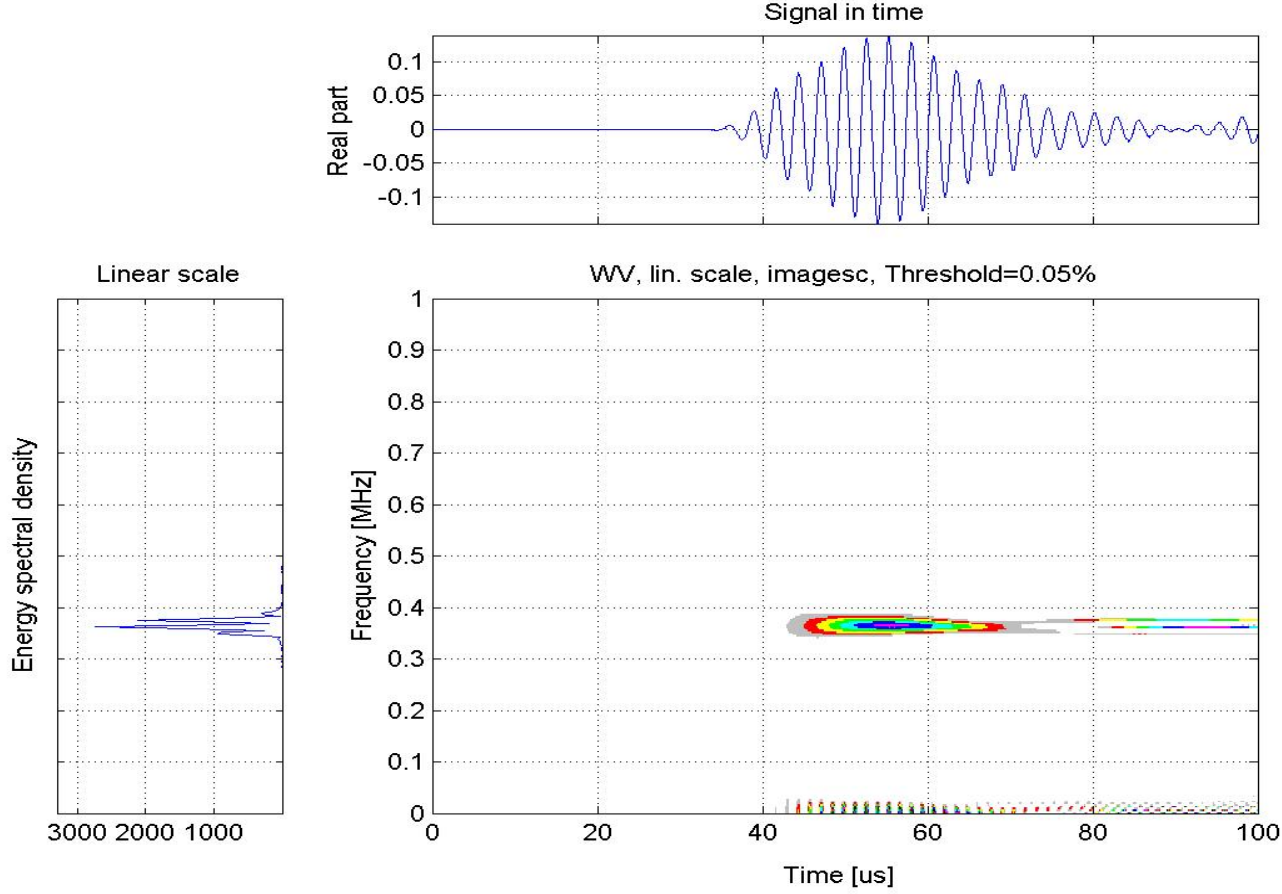

Figure 5.9 The Wigner-Ville Transform of $364 \mathrm{kHz}$ sine wave experiment output on the short $\operatorname{rod}$
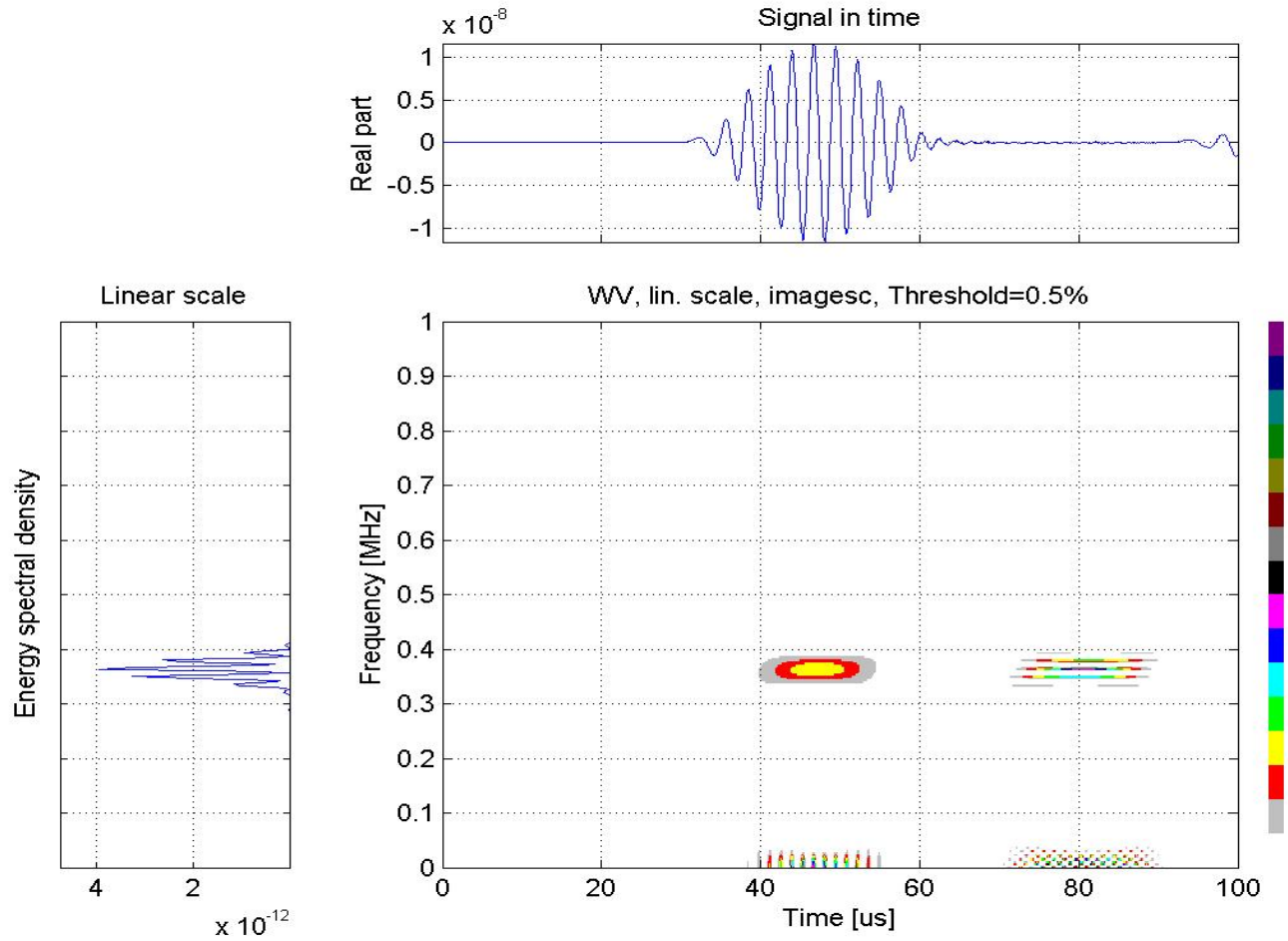

Figure 5.10 The Wigner-Ville Transform of $364 \mathrm{kHz}$ sine wave FEM output on the short rod 

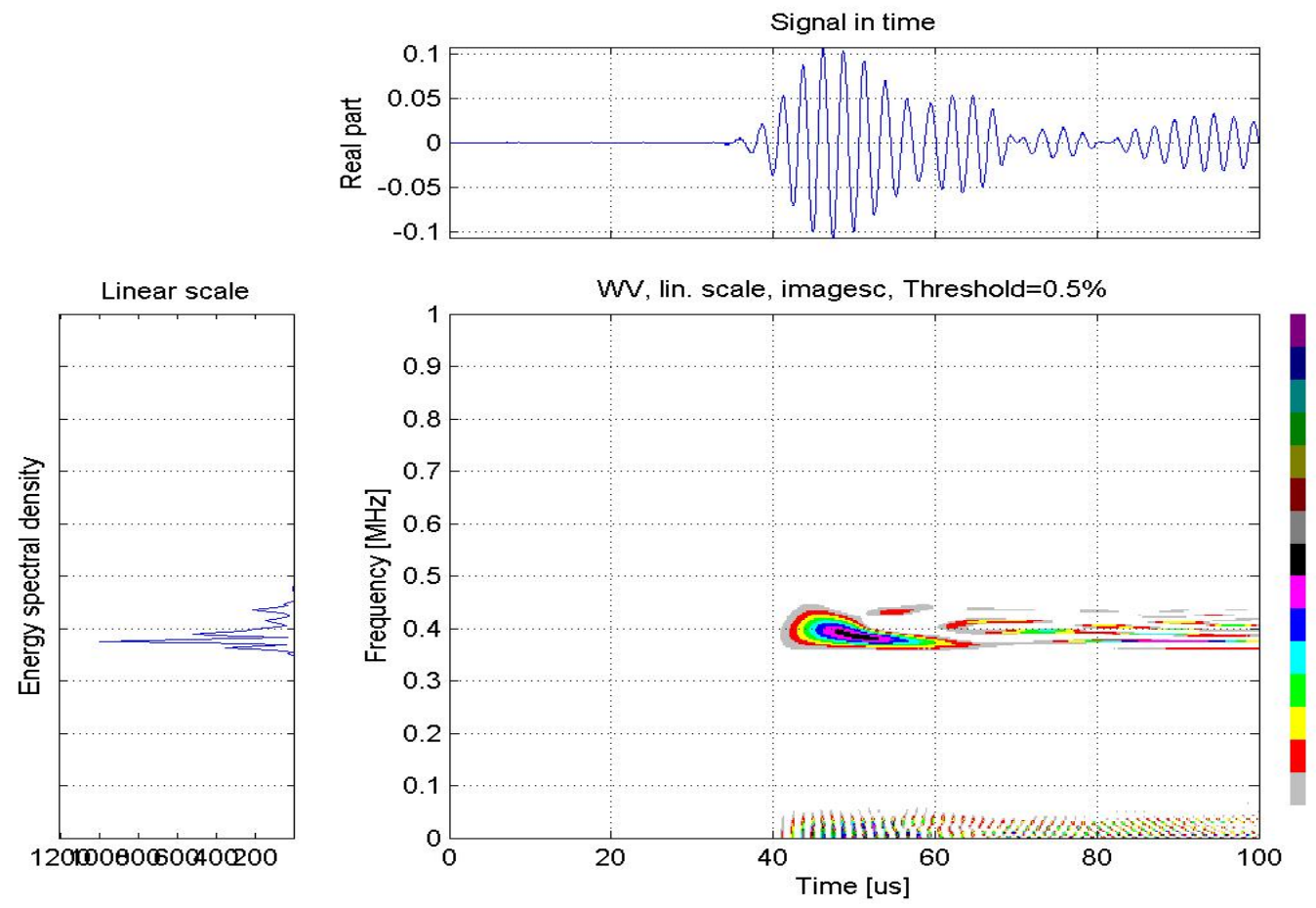

Figure 5.11 The Wigner-Ville Transform of $400 \mathrm{kHz}$ sine wave experiment output on the short rod
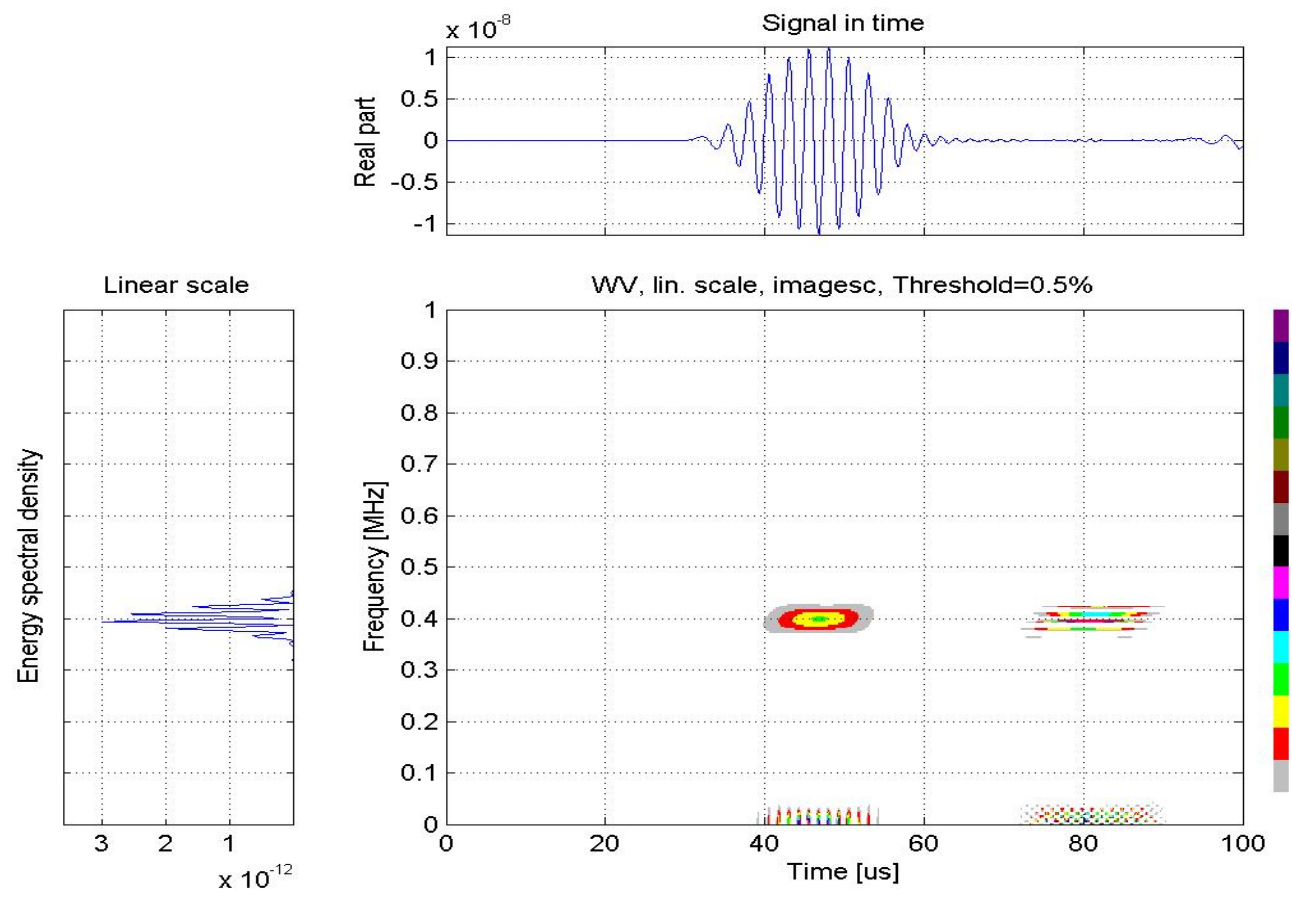

Figure 5.12 The Wigner-Ville Transform of $400 \mathrm{kHz}$ sine wave FEM output on the short rod 

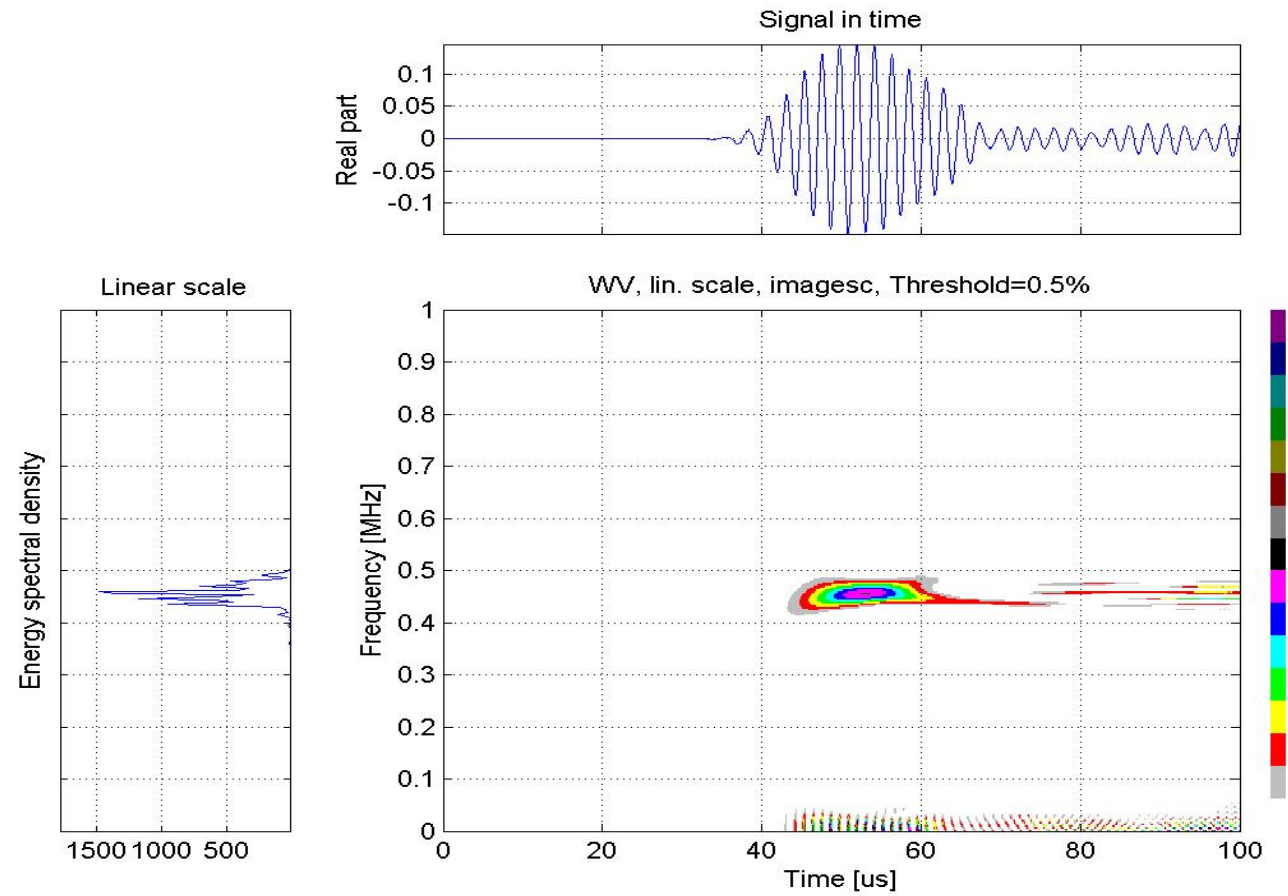

Figure 5.13 The Wigner-Ville Transform of $444 \mathrm{kHz}$ sine wave experiment output on the short rod
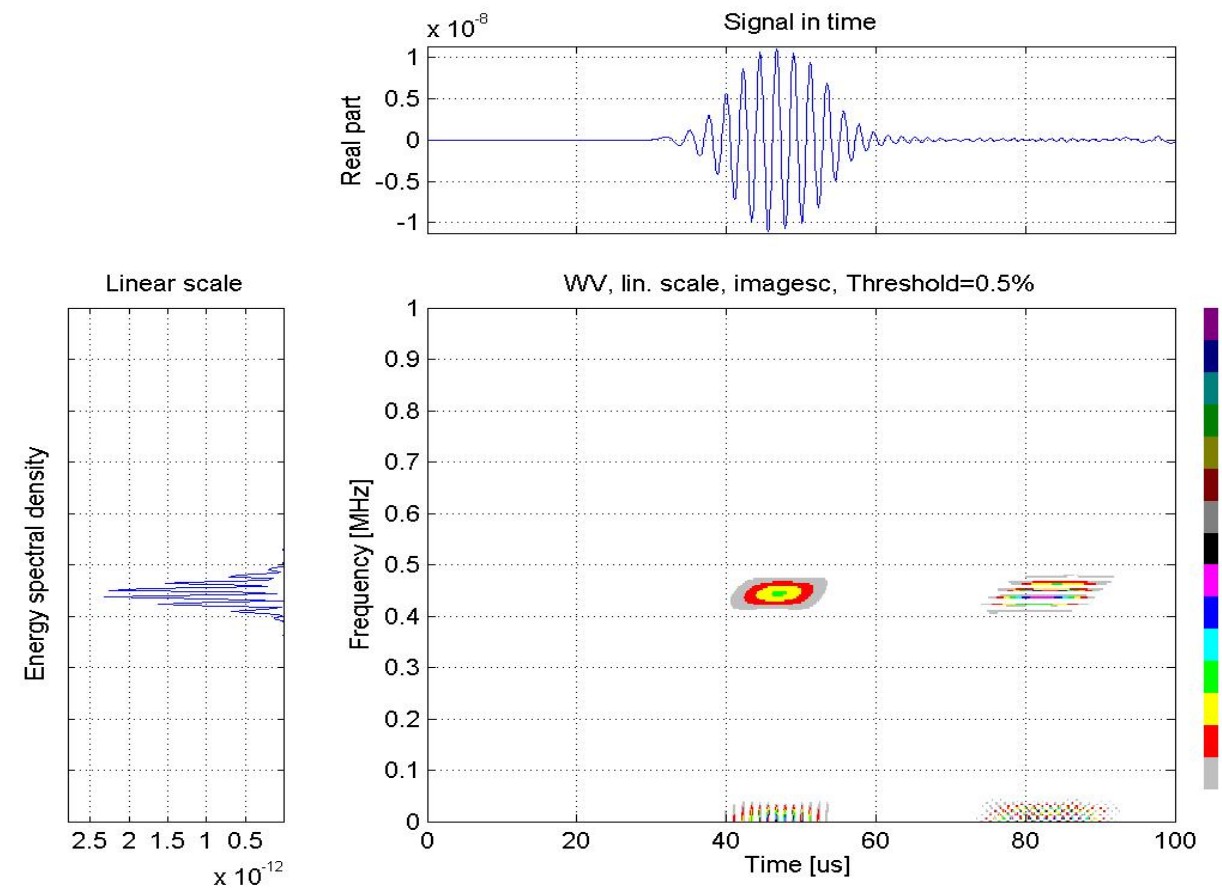

Figure 5.14 The Wigner-Ville Transform of $444 \mathrm{kHz}$ sine wave FEM output on the short rod 

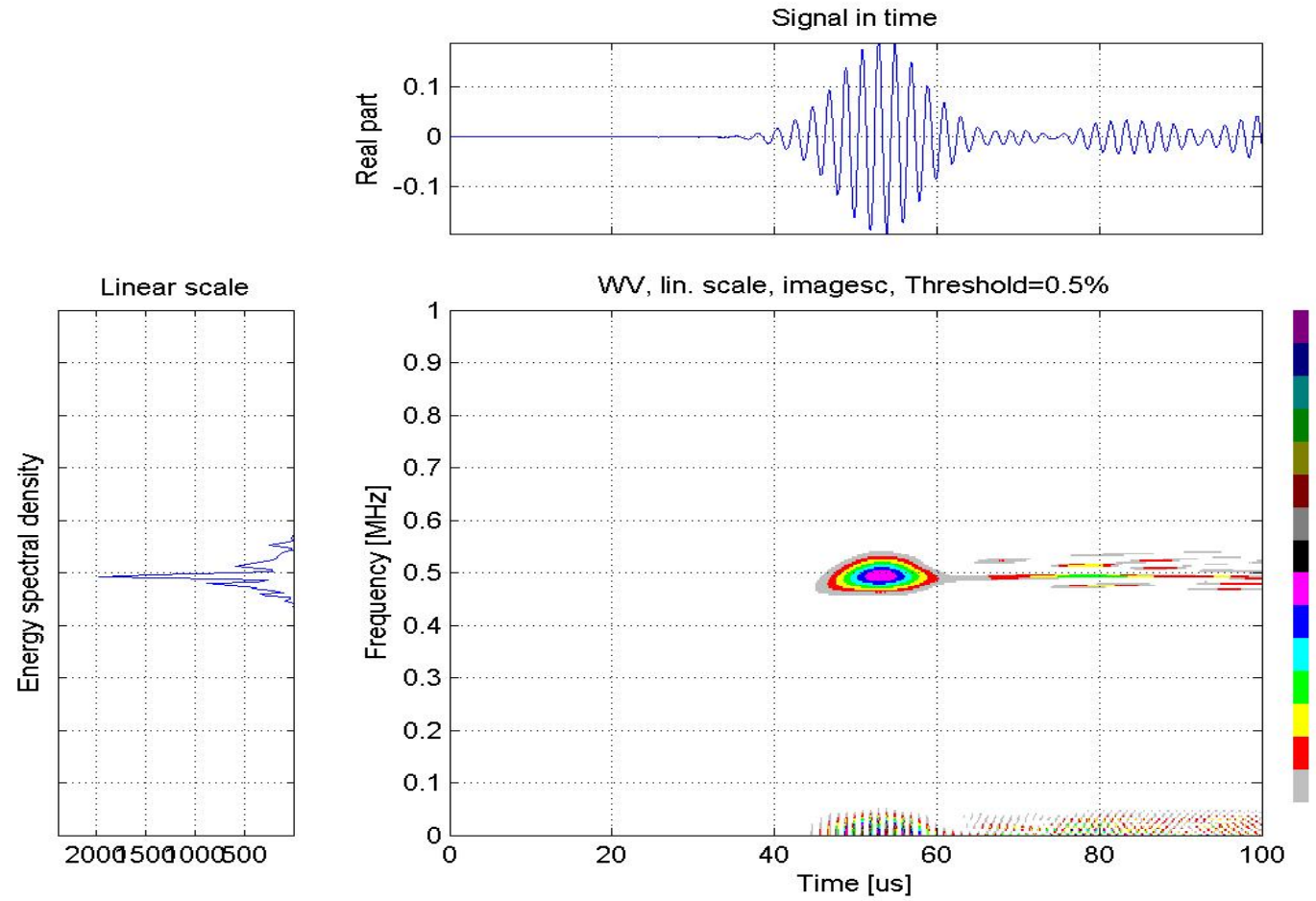

Figure 5.15 The Wigner-Ville Transform of $500 \mathrm{kHz}$ sine wave experiment output on the short rod
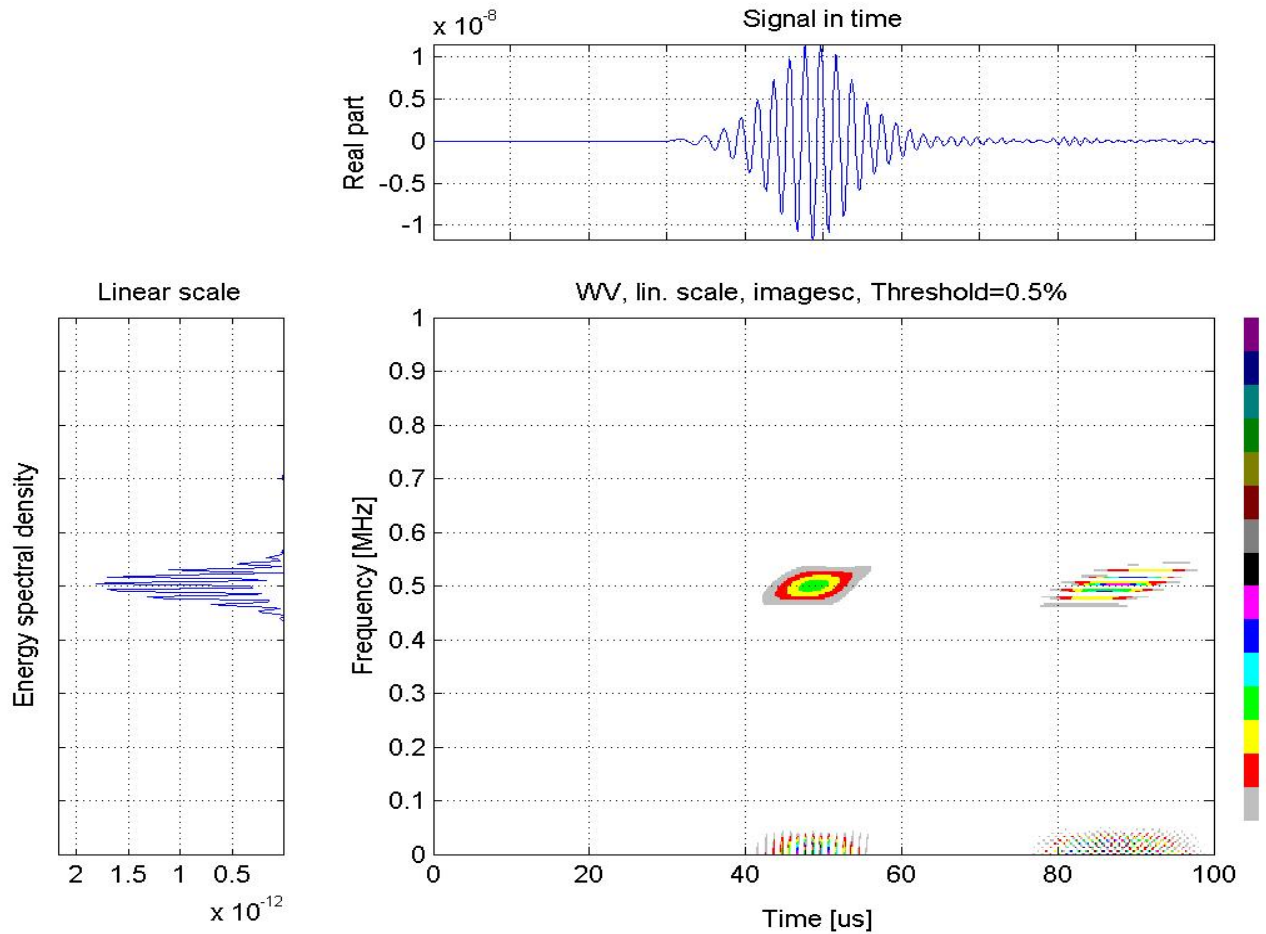

Figure 5.16 The Wigner-Ville Transform of $500 \mathrm{kHz}$ sine wave FEM output on the short rod 

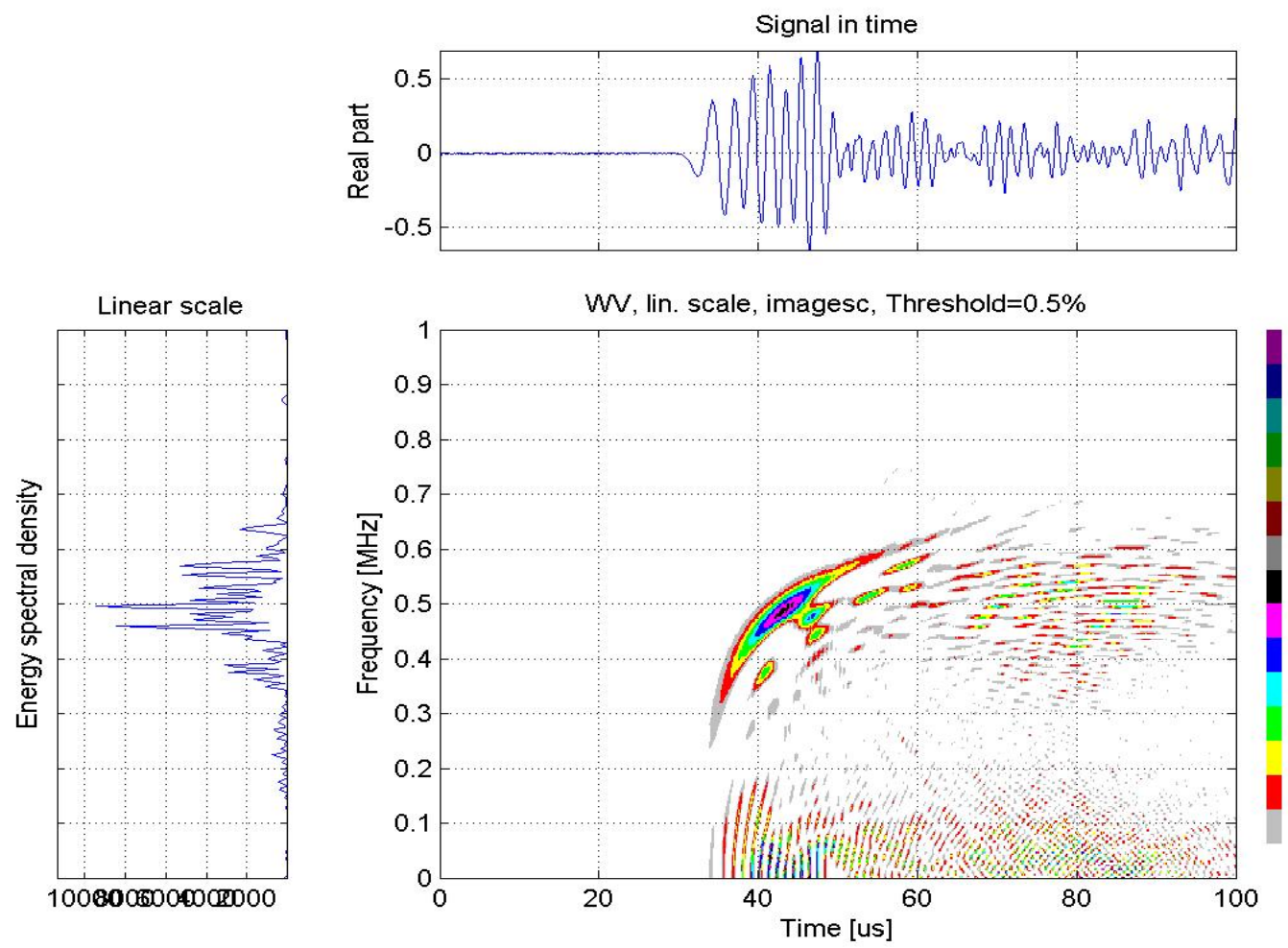

Figure 5.17 The Wigner-Ville Transform of pulse experiment output on the short rod
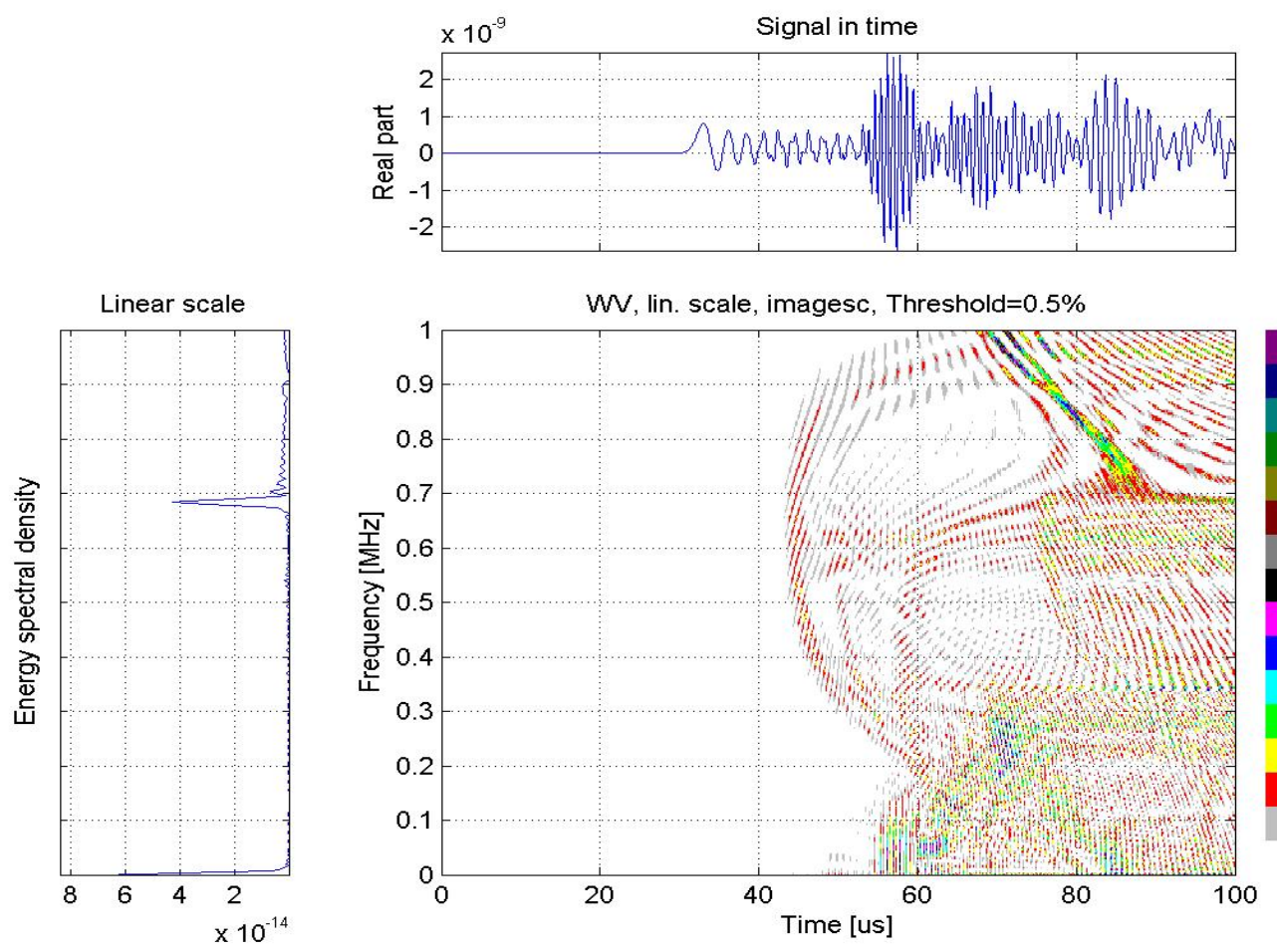

Figure 5.18 The Wigner-Ville Transform of pulse FEM output on the short rod 


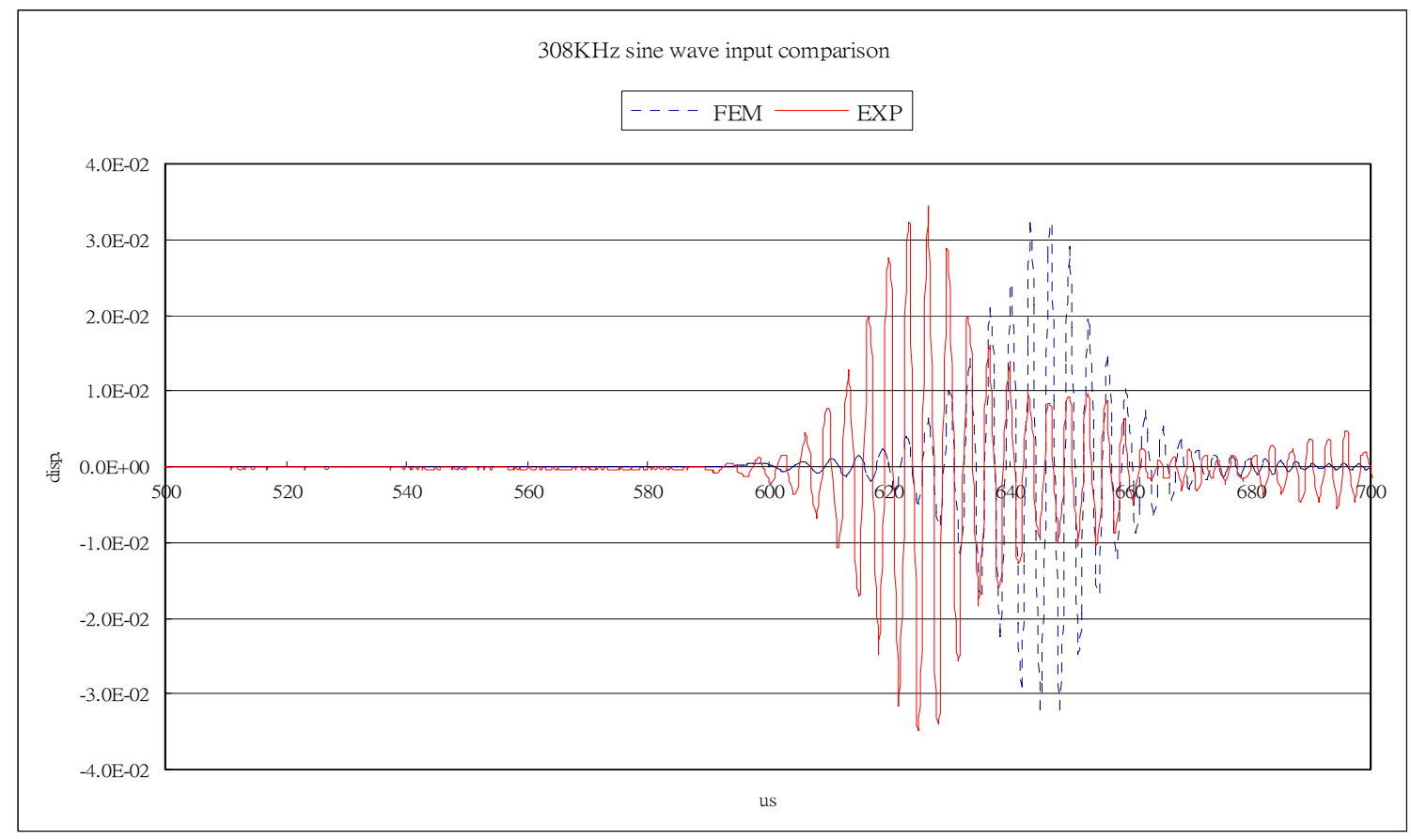

Figure 5.19 The waveform comparison between the experimental and FEM result from $308 \mathrm{kHz}$ sine wave input on the 3 meters rod

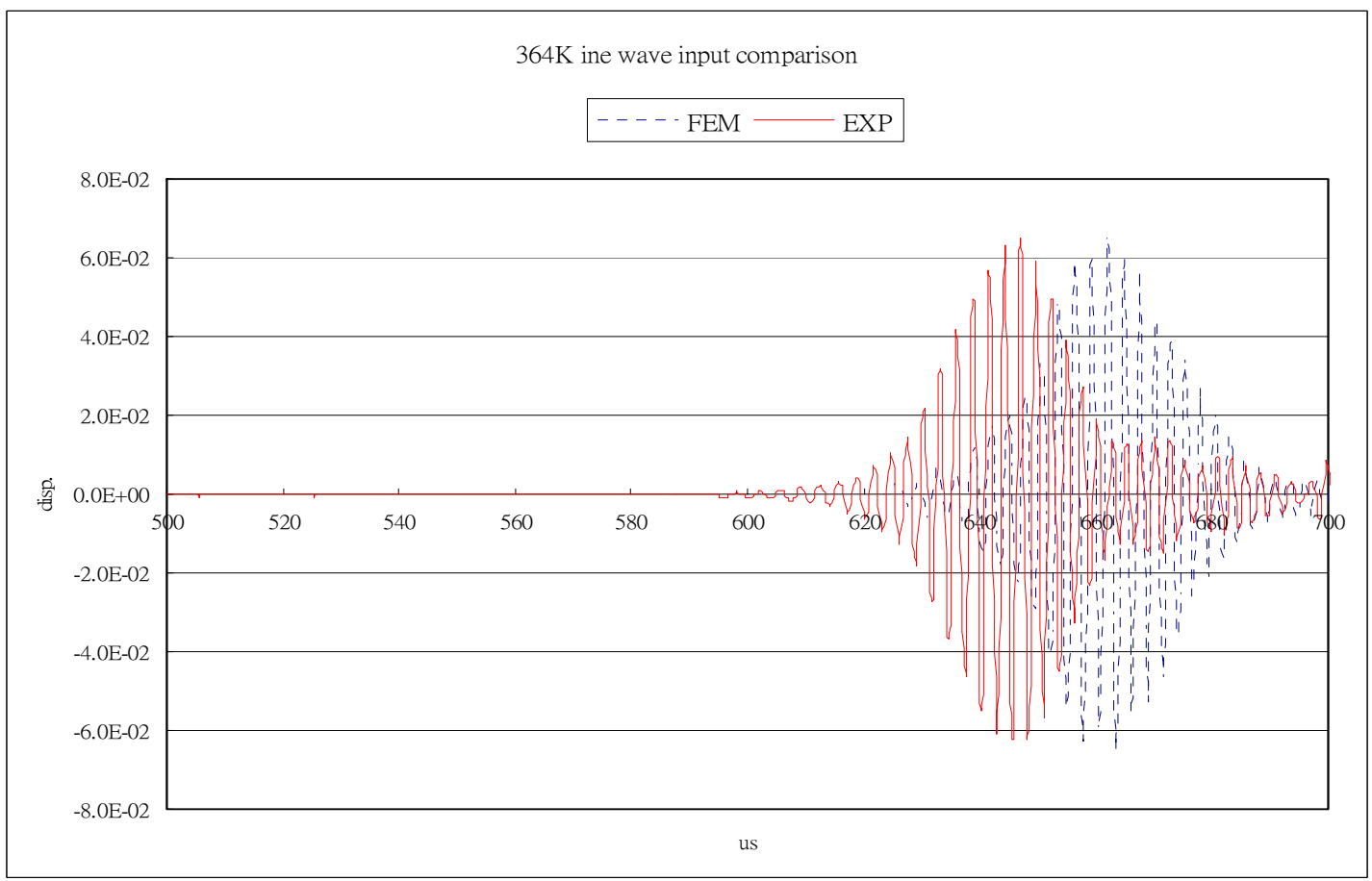

Figure 5.20 The waveform comparison between the experimental and FEM result from $364 \mathrm{kHz}$ sine wave input on the 3 meters rod 


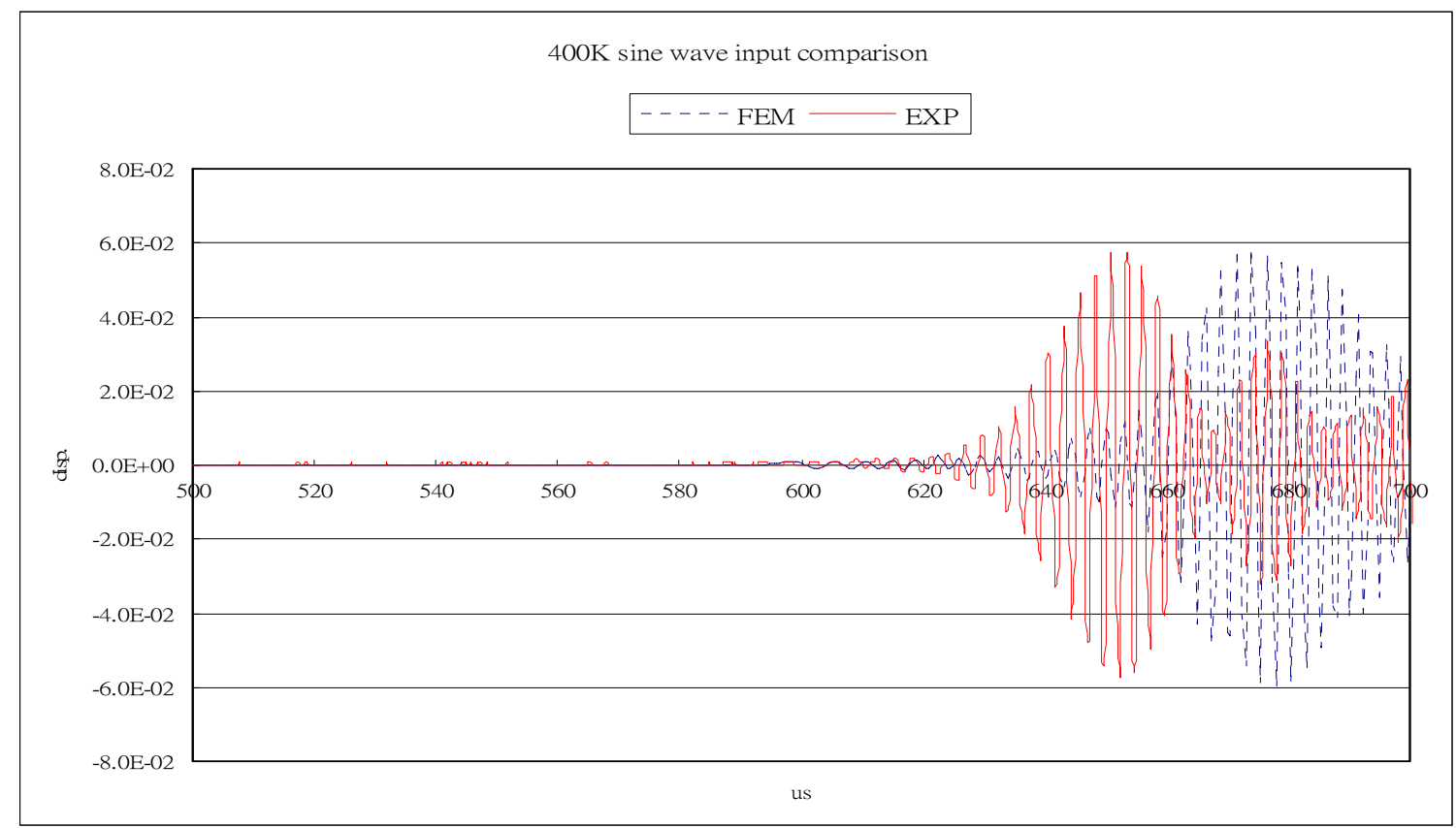

Figure 5.21 The waveform comparison between the experimental and FEM result

from $400 \mathrm{kHz}$ sine wave input on the 3 meters rod

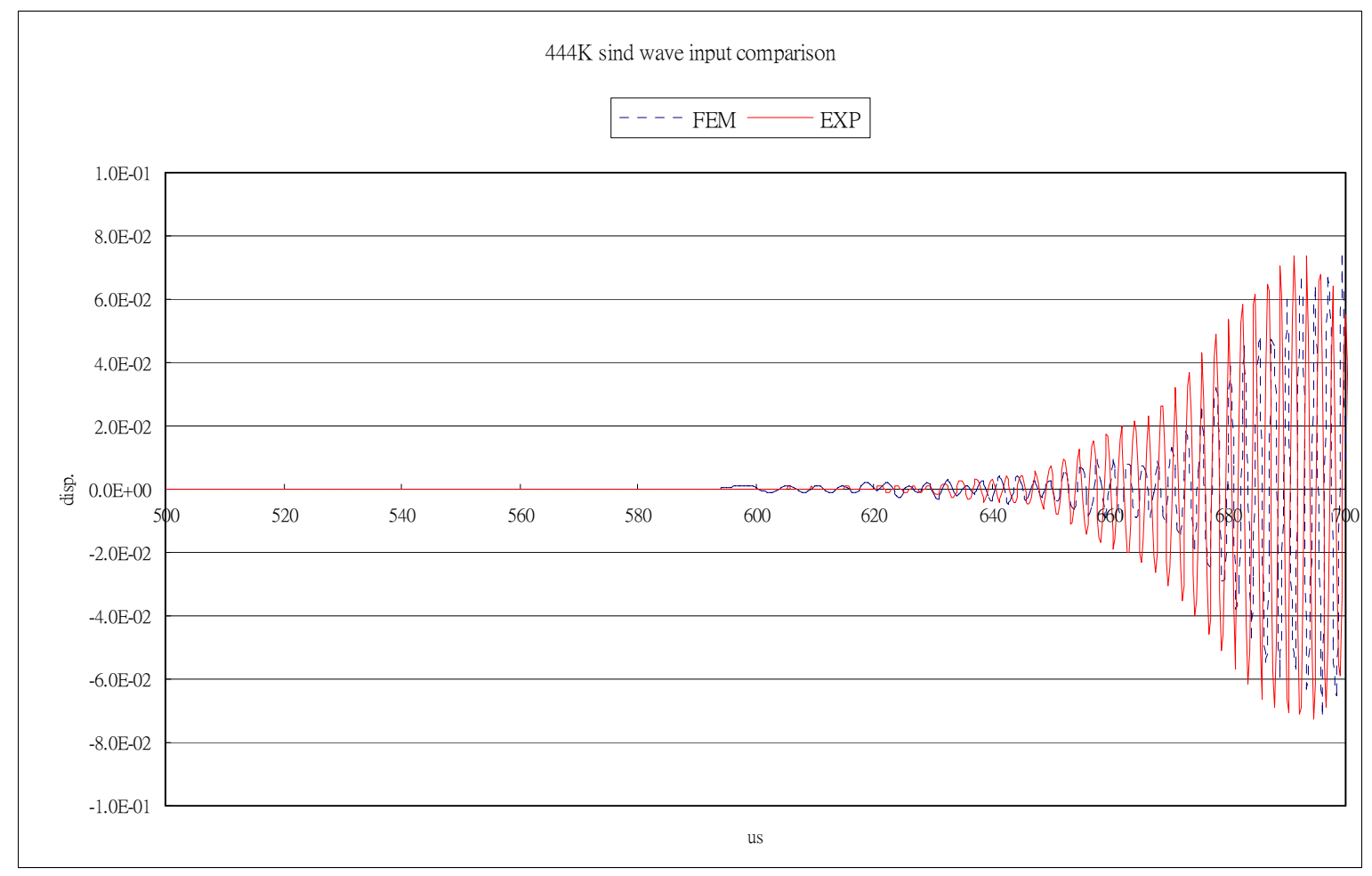

Figure 5.22 The waveform comparison between the experimental and FEM result from $444 \mathrm{kHz}$ sine wave input on the 3 meters rod 


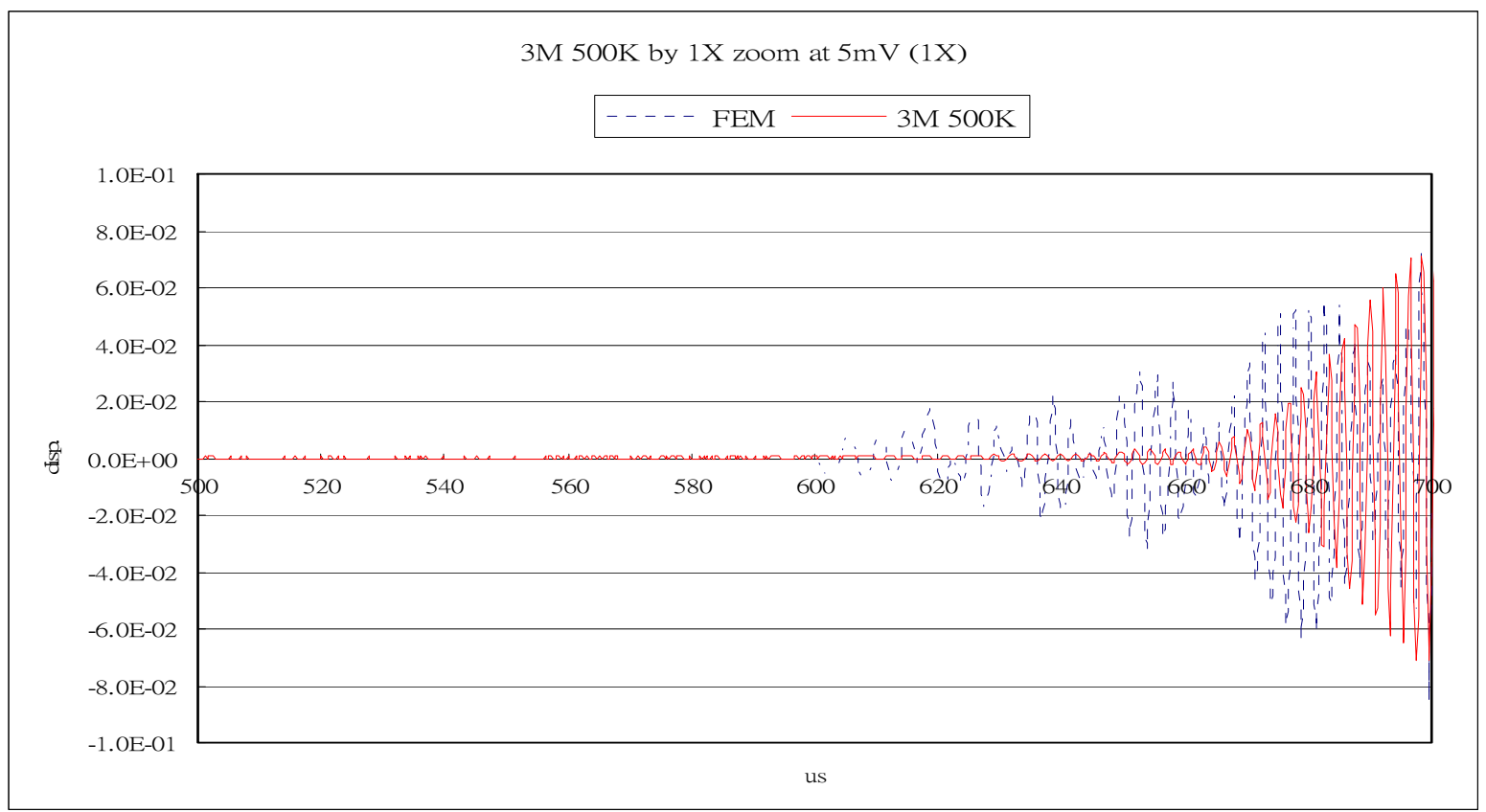

Figure 5.23 The waveform comparison between the experimental and FEM result from 500 $\mathrm{kHz}$ sine wave input on the 3 meters rod 


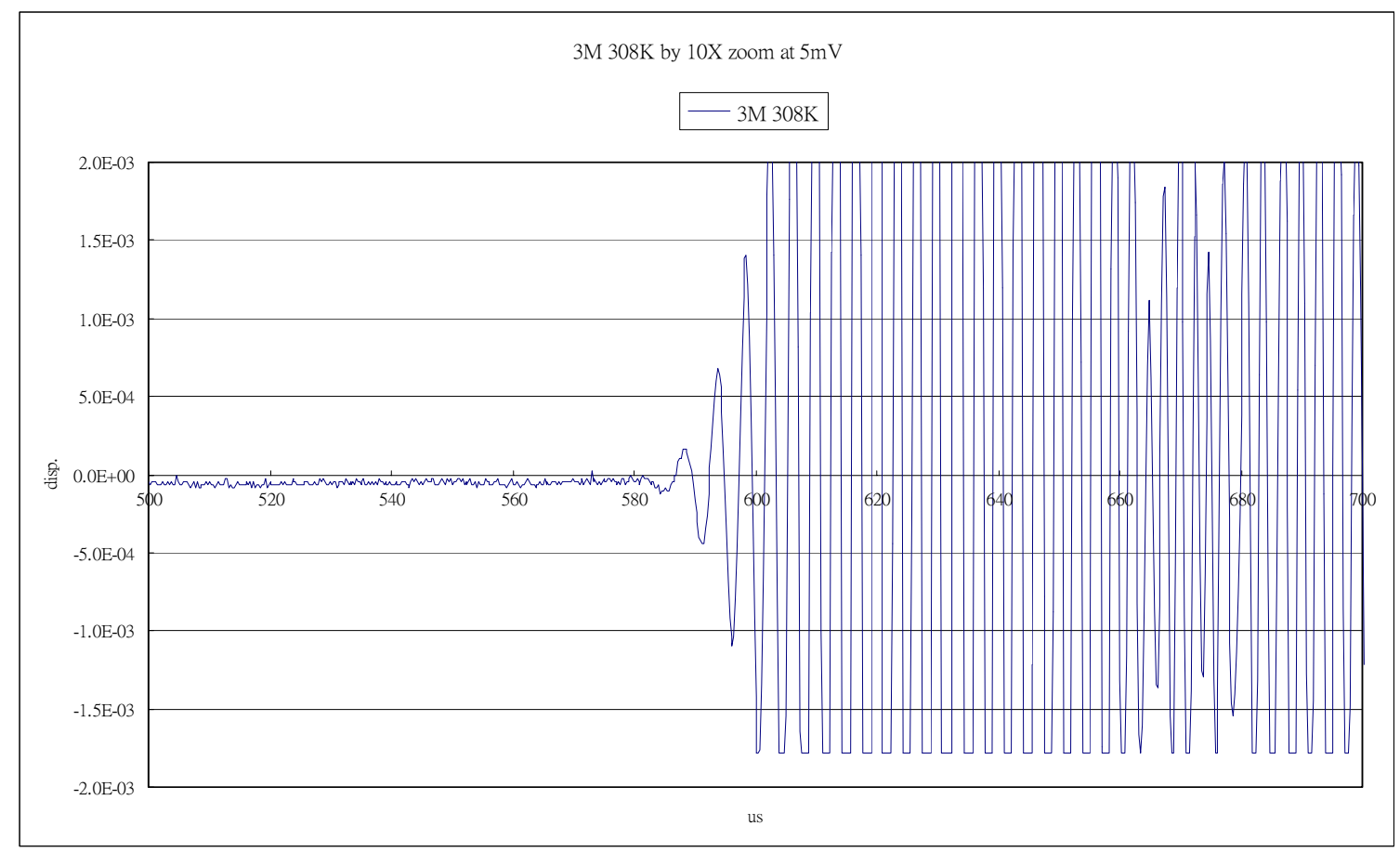

Figure 5.24 The zoom-in experimental waveform from $308 \mathrm{kHz}$ sine wave input on the 3 meters rod

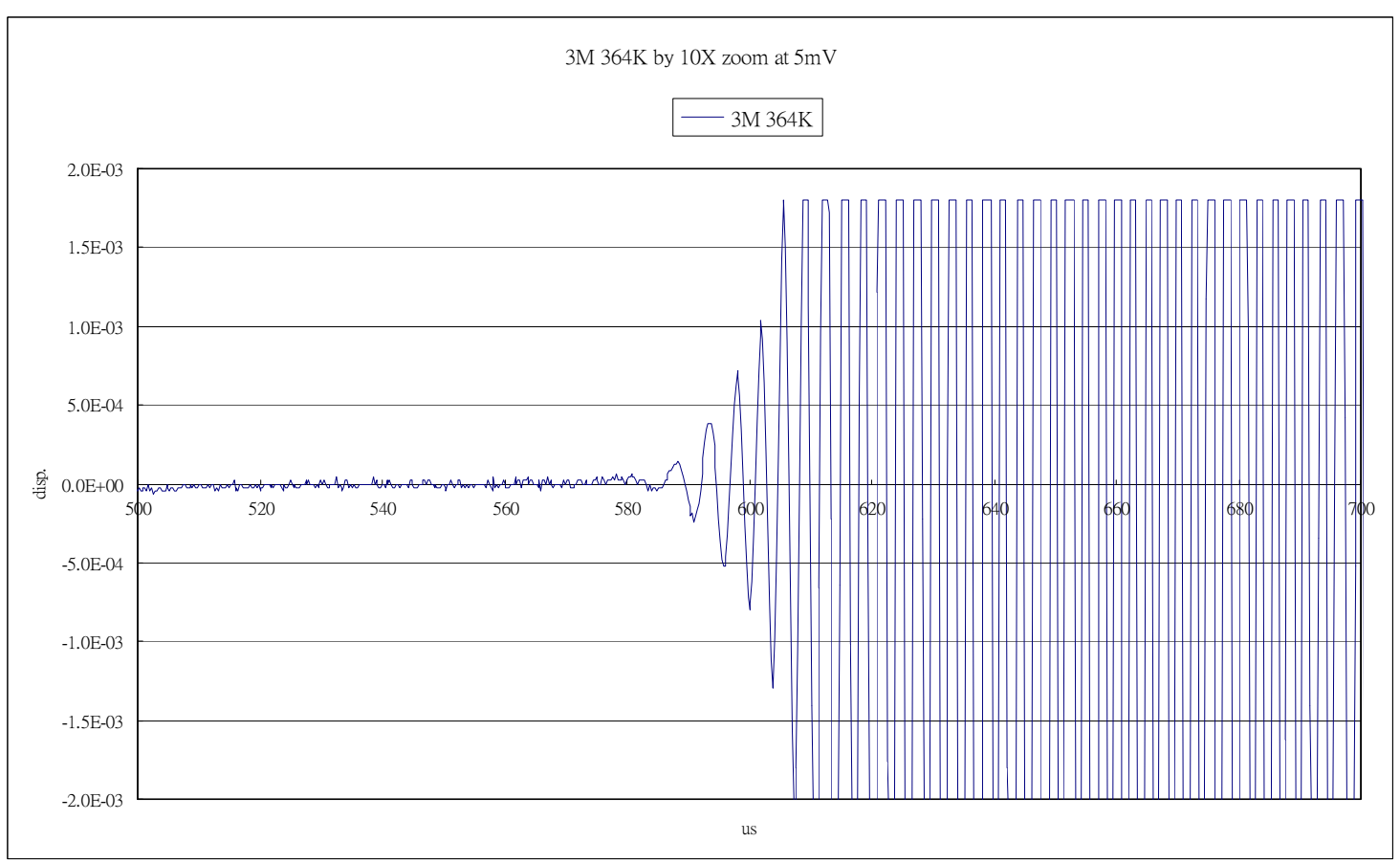

Figure 5.25 The zoom-in experimental waveform from $364 \mathrm{kHz}$ sine wave input on the 3 meters rod 


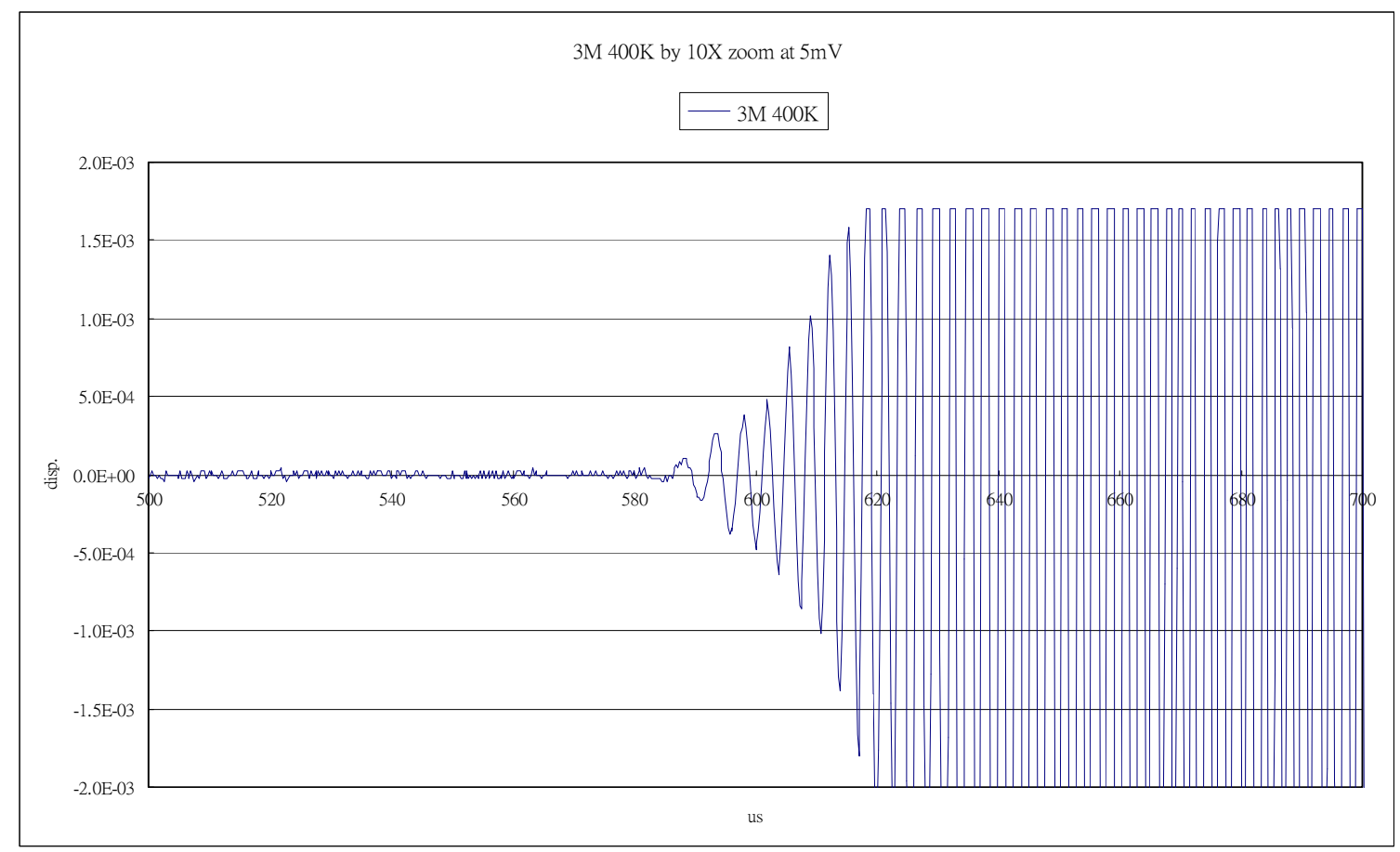

Figure 5.26 The zoom-in experimental waveform from $400 \mathrm{kHz}$ sine wave input on the 3 meters rod

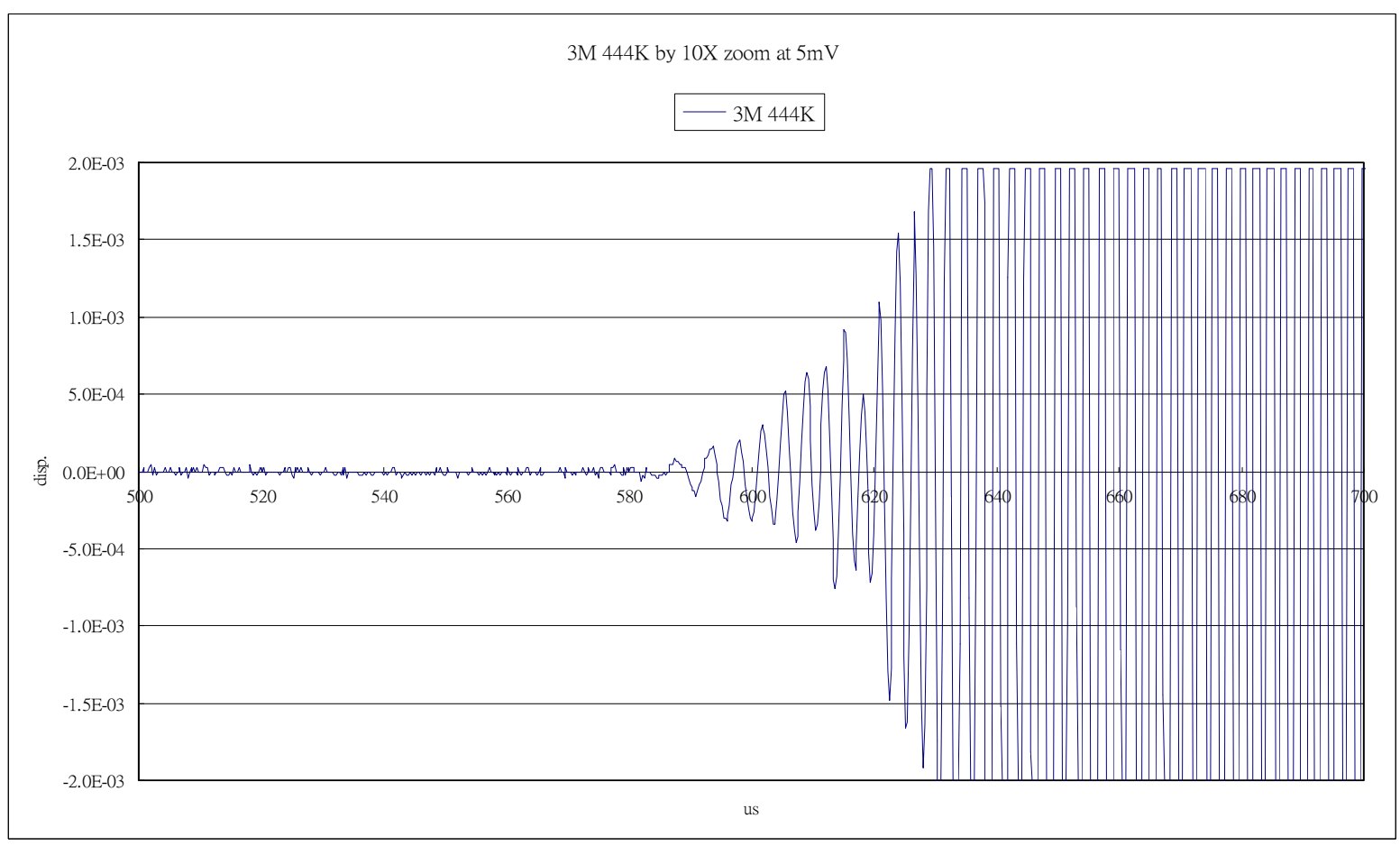

Figure 5.27 The zoom-in experimental waveform from $444 \mathrm{kHz}$ sine wave input on the 3 meters rod 


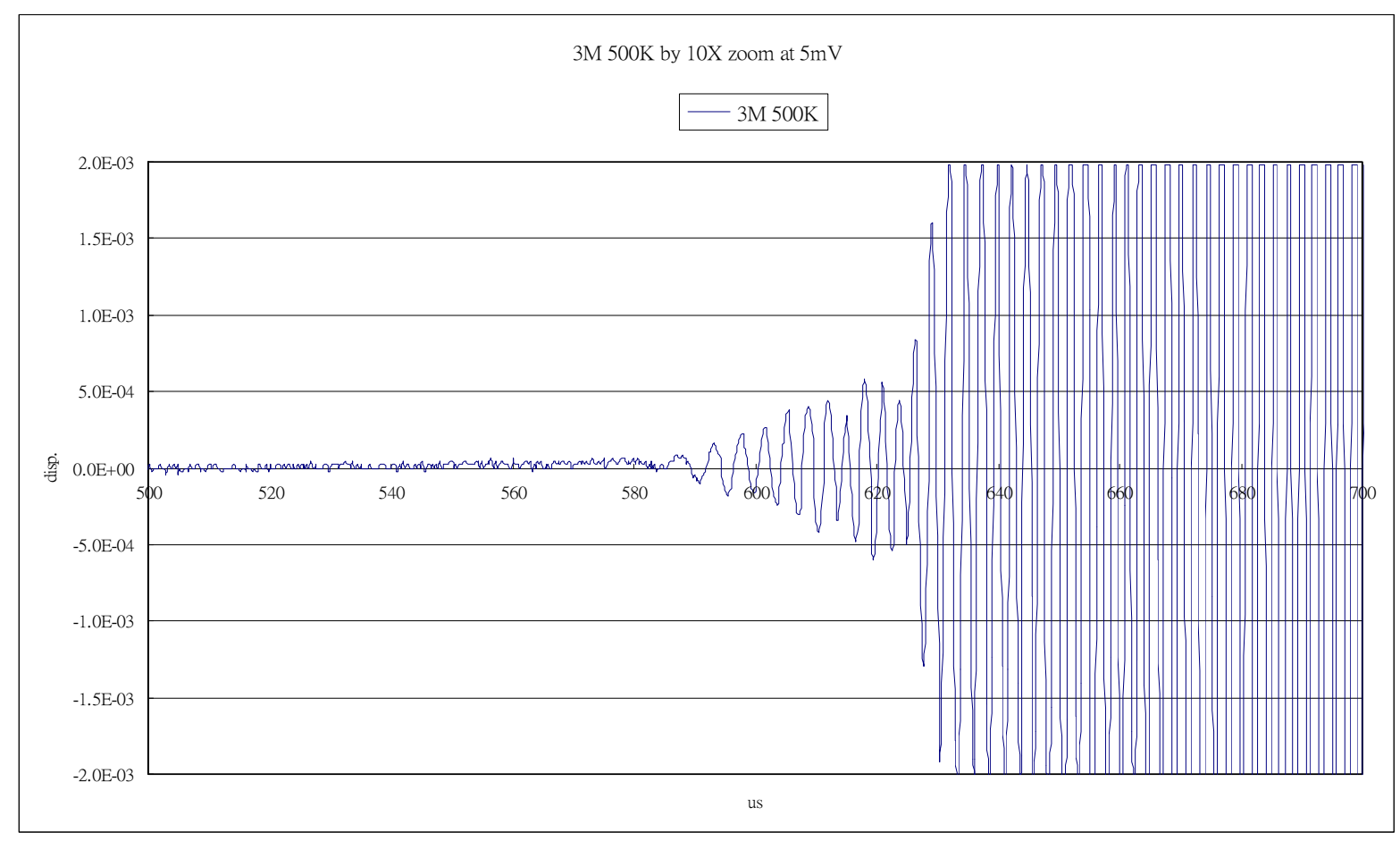

Figure 5.28 The zoom-in experimental waveform from $500 \mathrm{kHz}$ sine wave input on the 3 meters rod

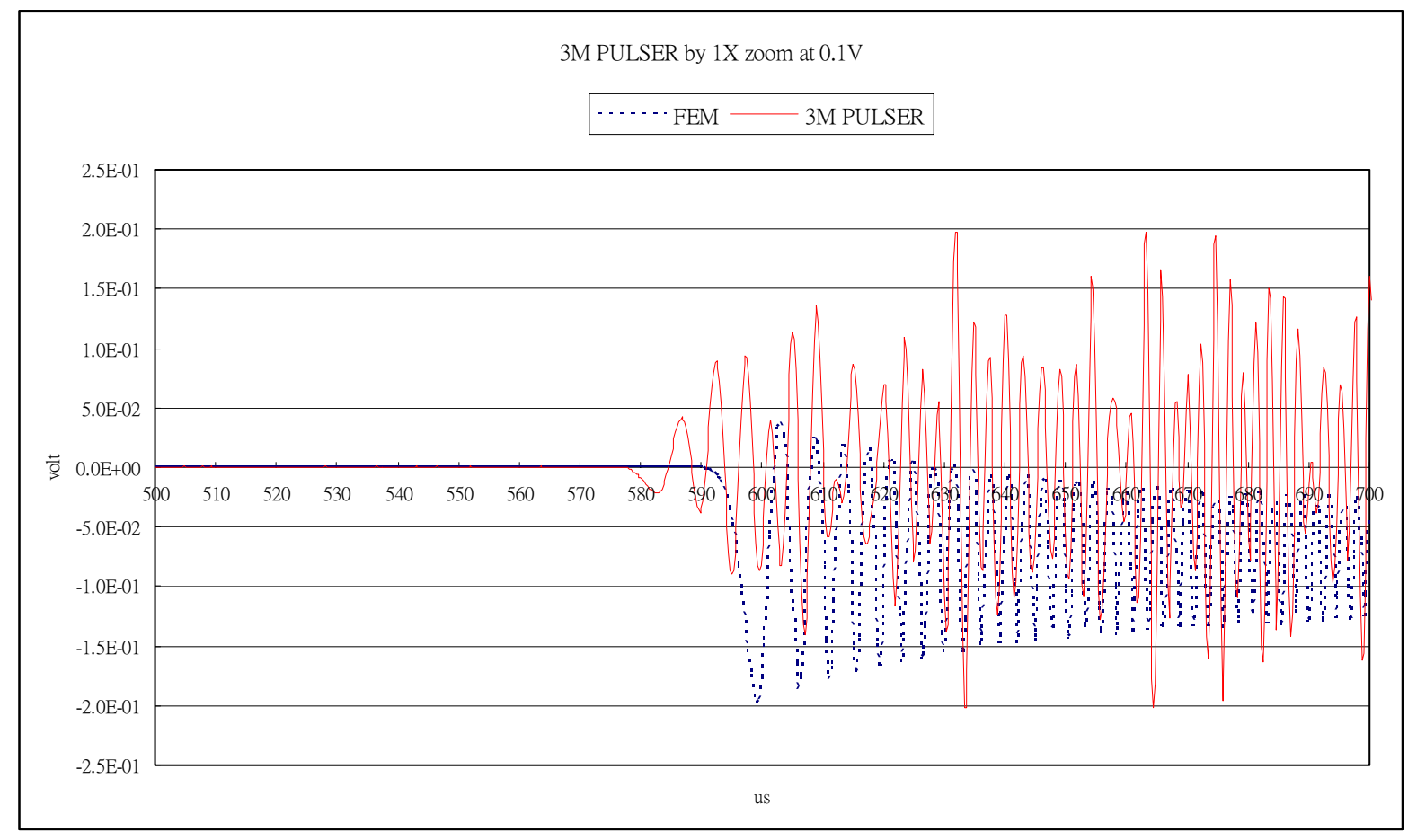

Figure 5.29 The waveform comparison between the experimental and FEM result from the C101HV pulse generator input on the 3 meters rod 

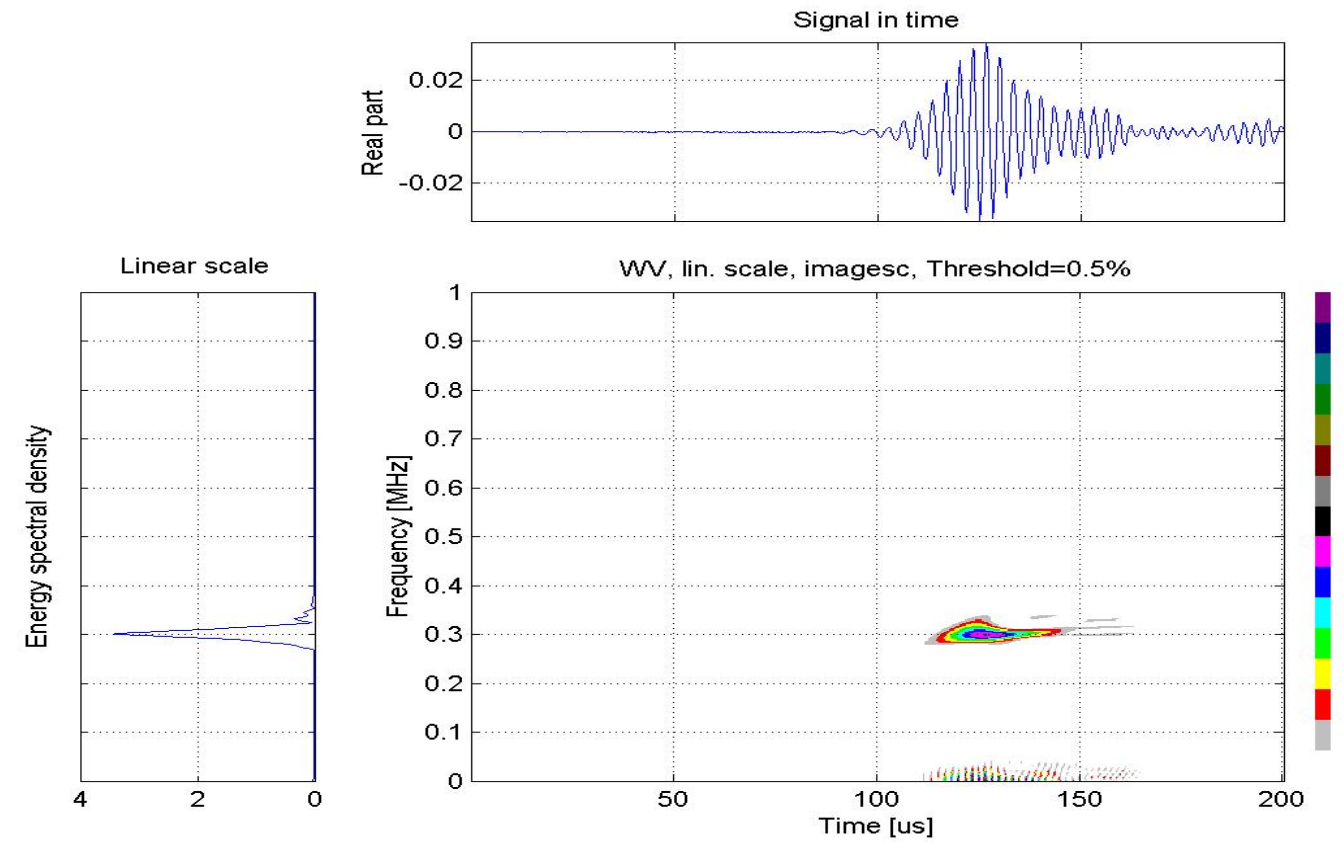

Figure 5.30 The Wigner-Ville Transform applied on experimental result due to the $308 \mathrm{kHz}$ sine wave input on the 3 meters rod
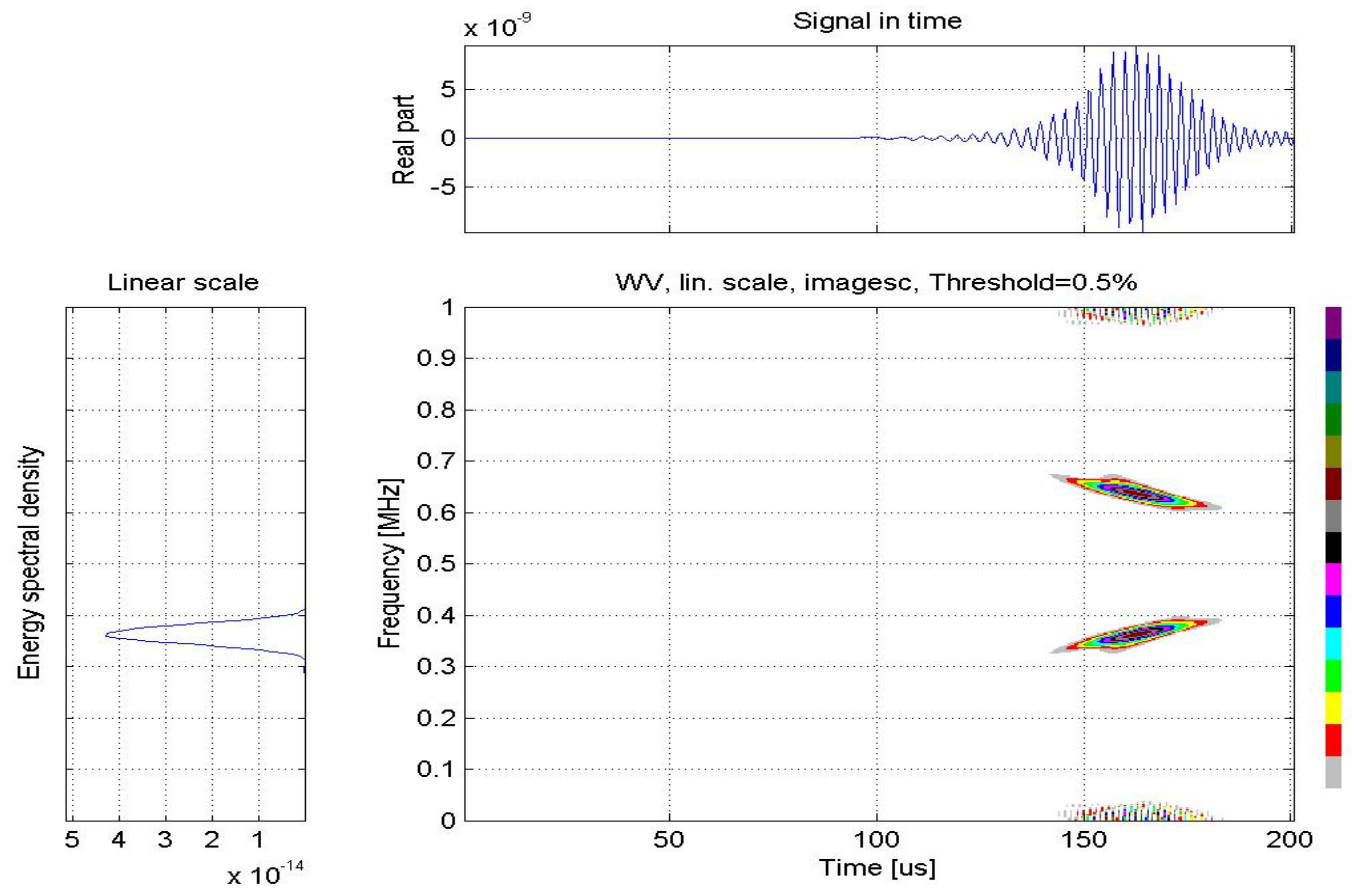

Figure 5.31 The Wigner-Ville Transform applied on FEM result due to the $308 \mathrm{kHz}$ sine wave input on the 3 meters rod 

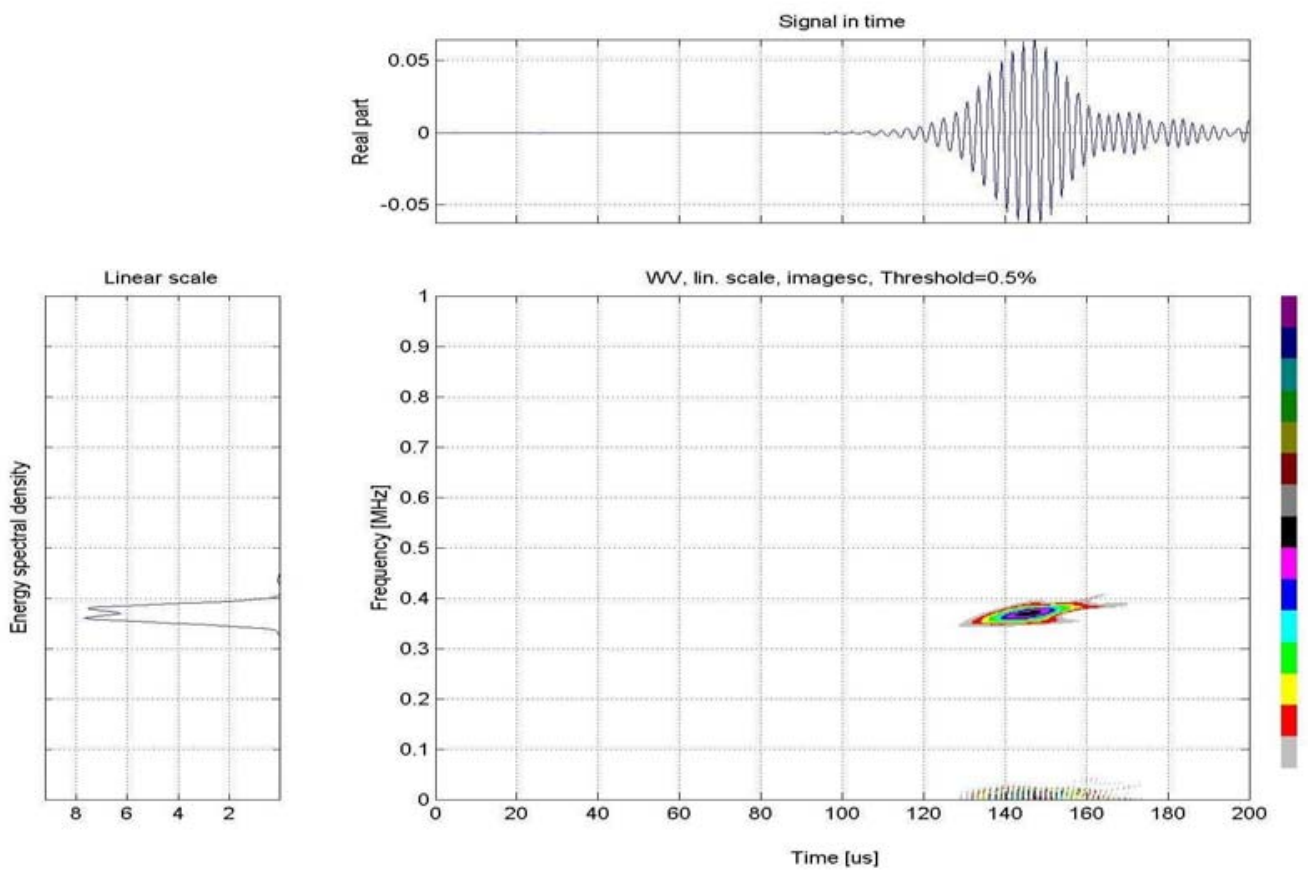

Figure 5.32 The Wigner-Ville Transform applied on experimental result due to the $364 \mathrm{kHz}$ sine wave input on the 3 meters rod
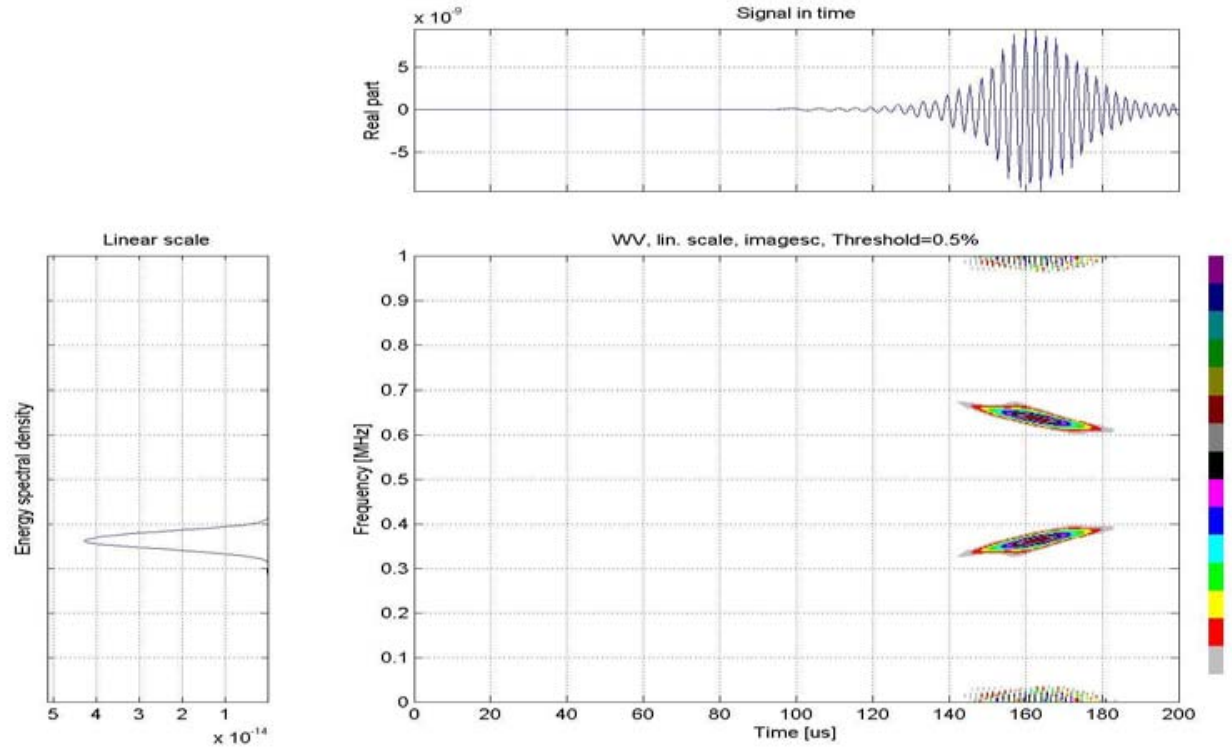

Figure 5.33 The Wigner-Ville Transform applied on FEM result due to the $364 \mathrm{kHz}$ sine wave input on the 3 meters rod 

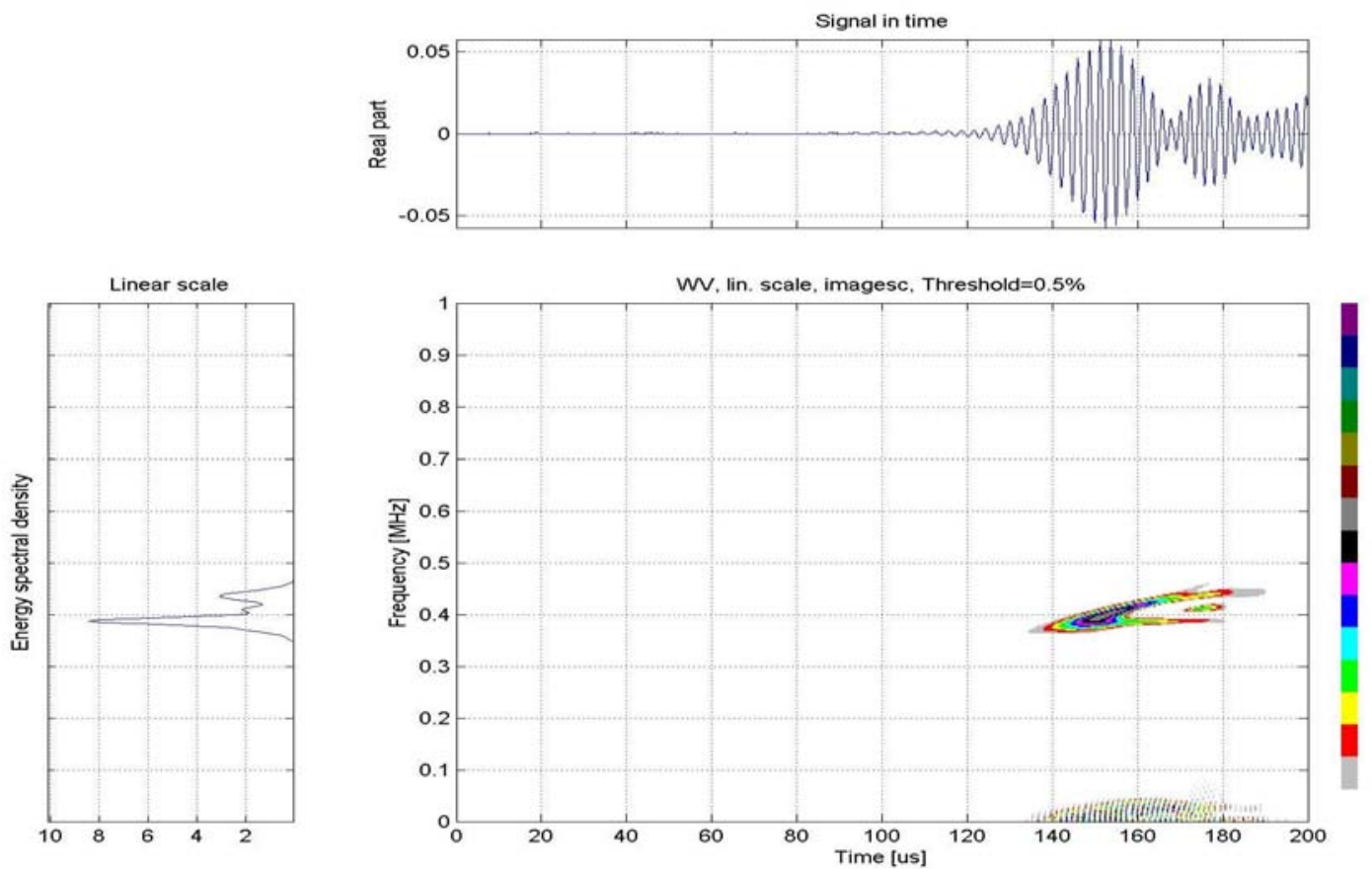

Figure 5.34 The Wigner-Ville Transform applied on experimental result due to the $400 \mathrm{kHz}$ sine wave input on the 3 meters rod
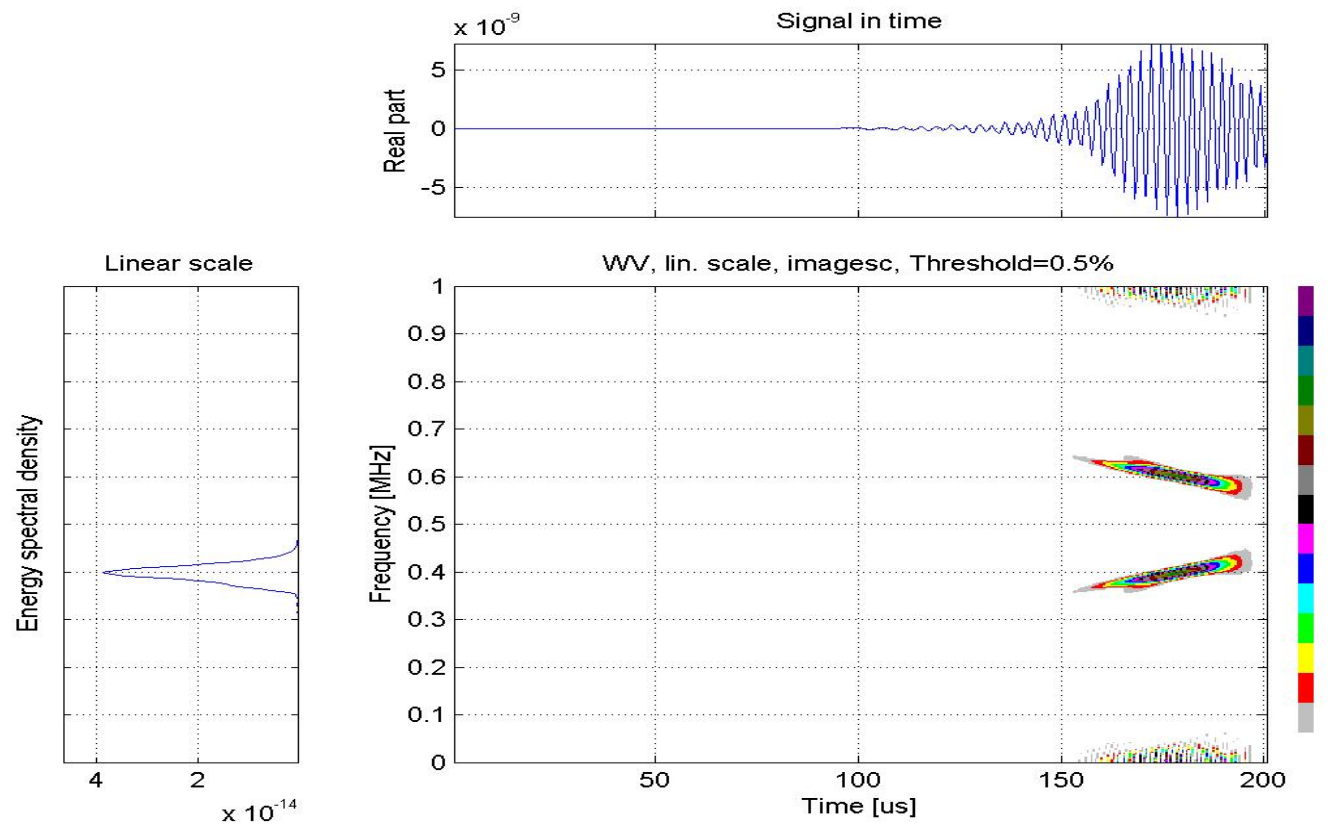

Figure 5.35 The Wigner-Ville Transform applied on FEM result due to the $400 \mathrm{kHz}$ sine wave input on the 3 meters rod 

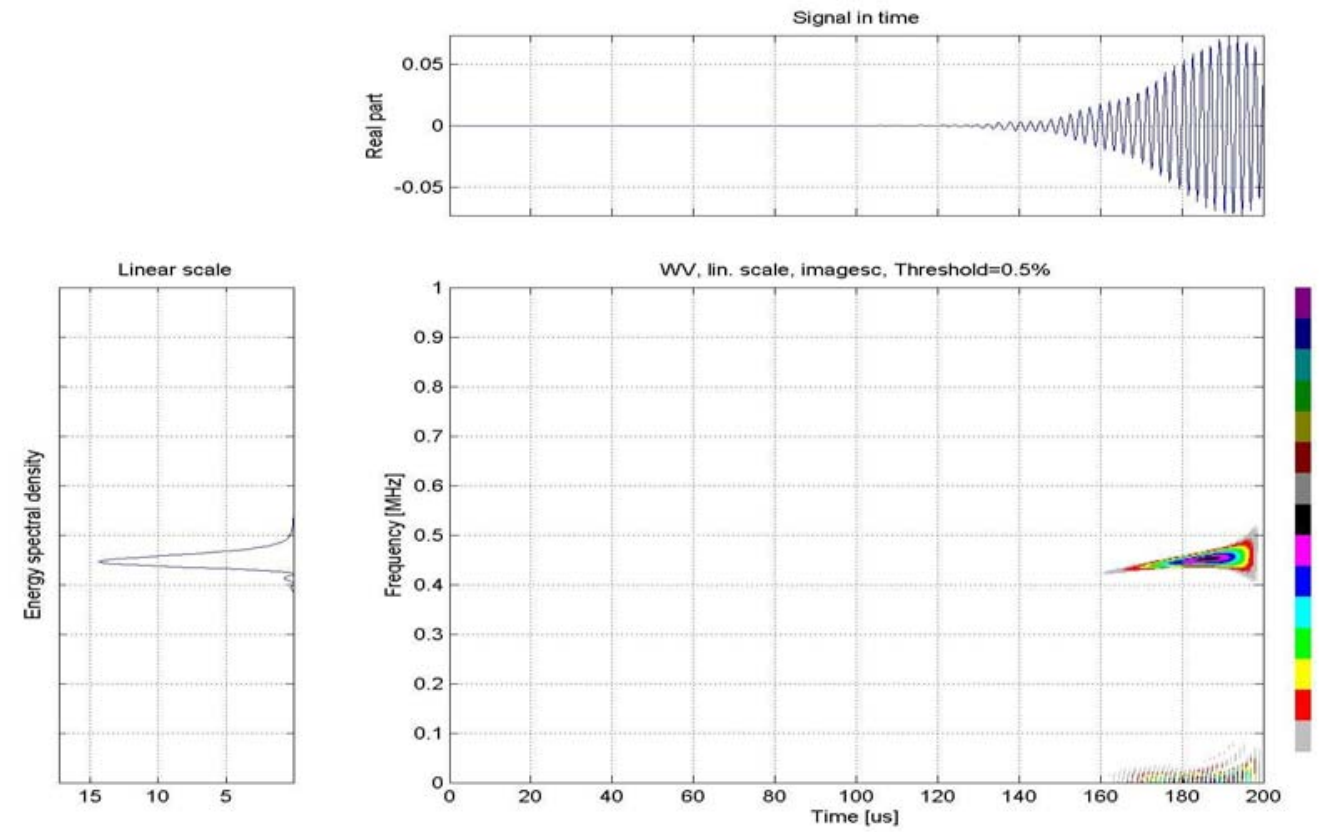

Figure 5.36 The Wigner-Ville Transform applied on experimental result due to the $444 \mathrm{kHz}$ sine wave input on the 3 meters rod
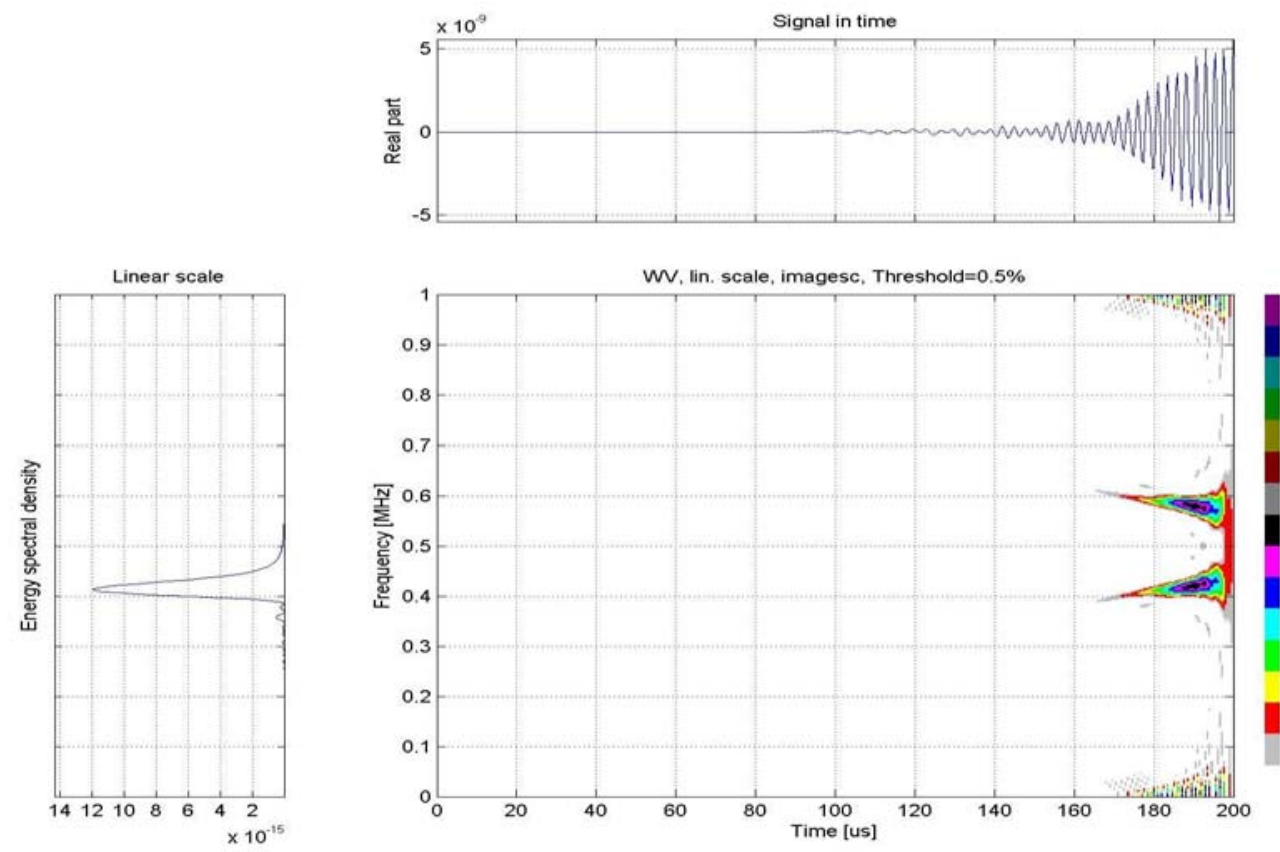

Figure 5.37 The Wigner-Ville Transform applied on FEM result due to the $444 \mathrm{kHz}$ sine wave input on the 3 meters rod 

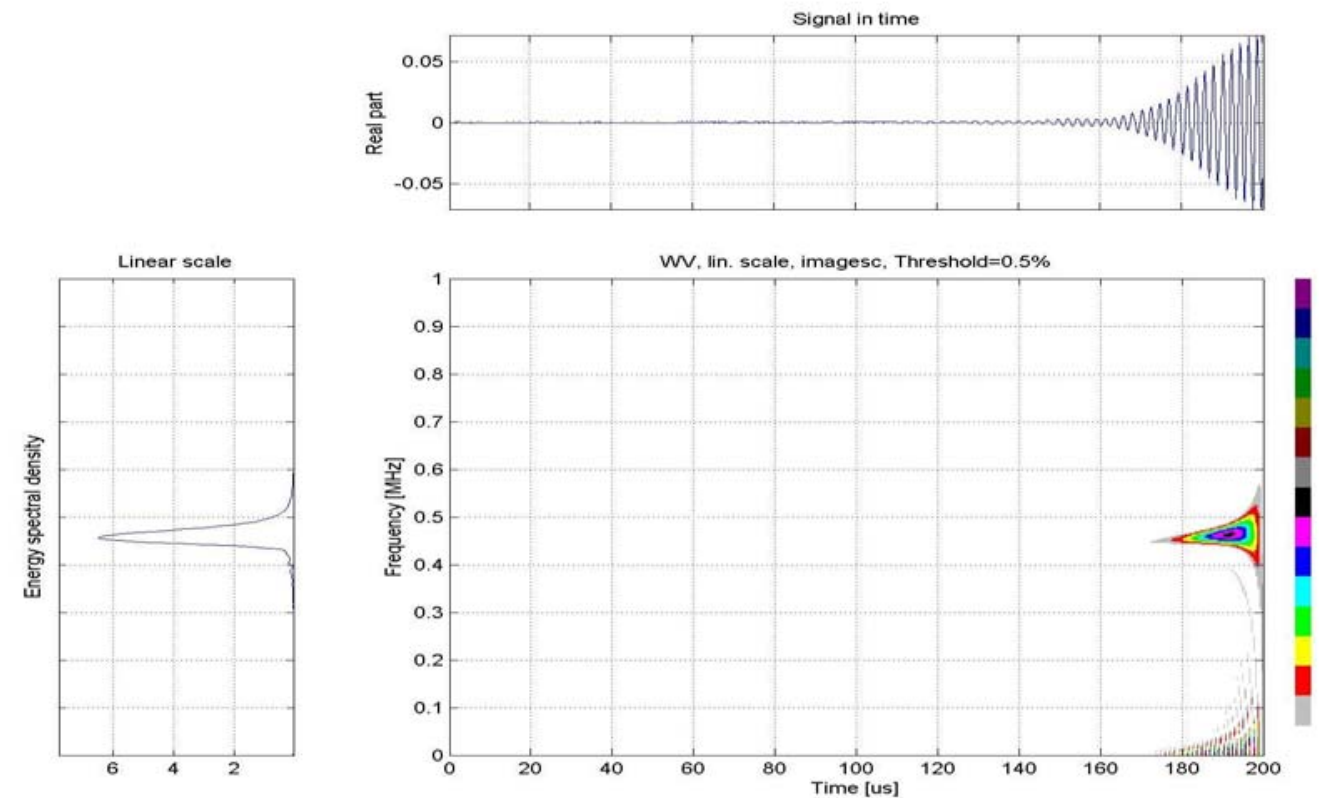

Figure 5.38 The Wigner-Ville Transform applied on experimental result due to the $500 \mathrm{kHz}$ sine wave input on the 3 meters rod
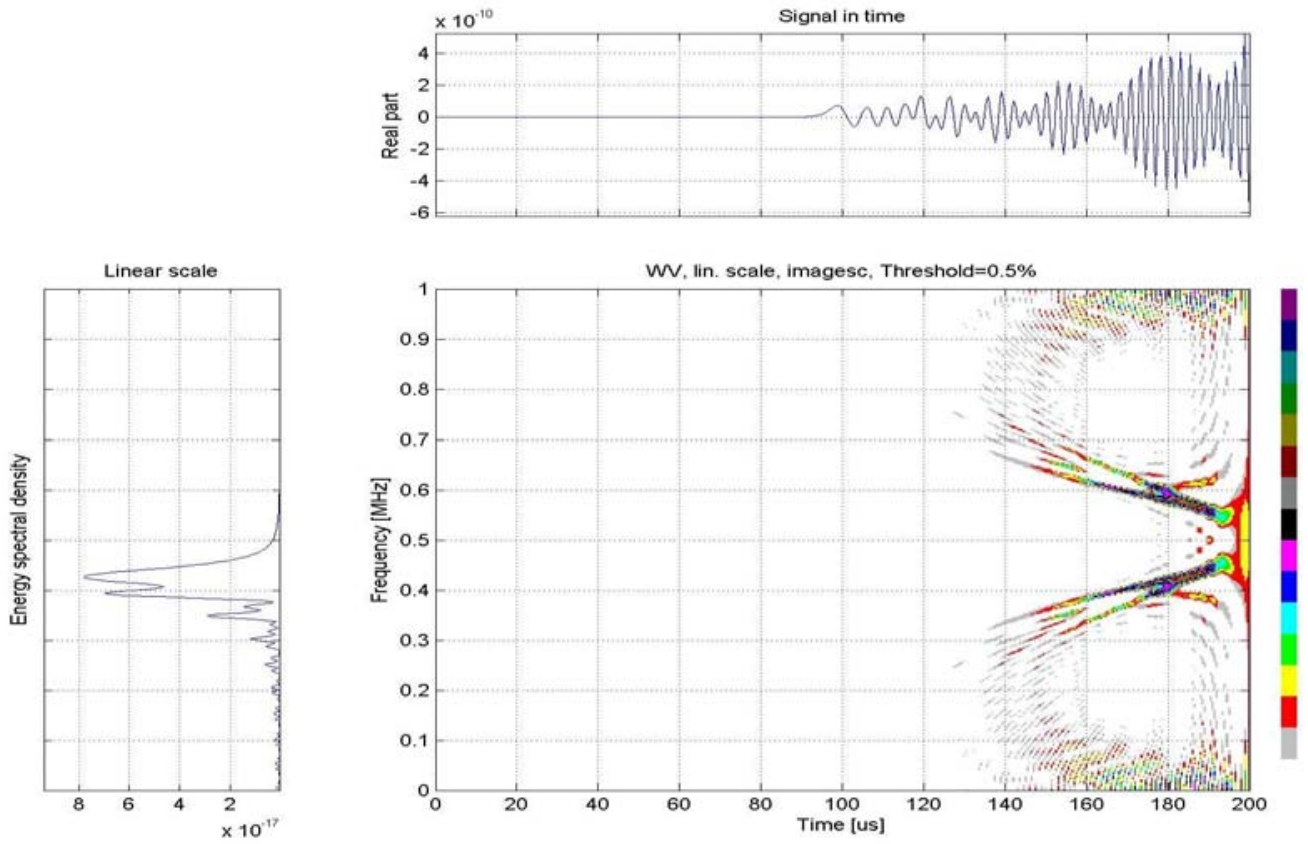

Figure 5.39 The Wigner-Ville Transform applied on FEM result due to the $500 \mathrm{kHz}$ sine wave input on the 3 meters rod 

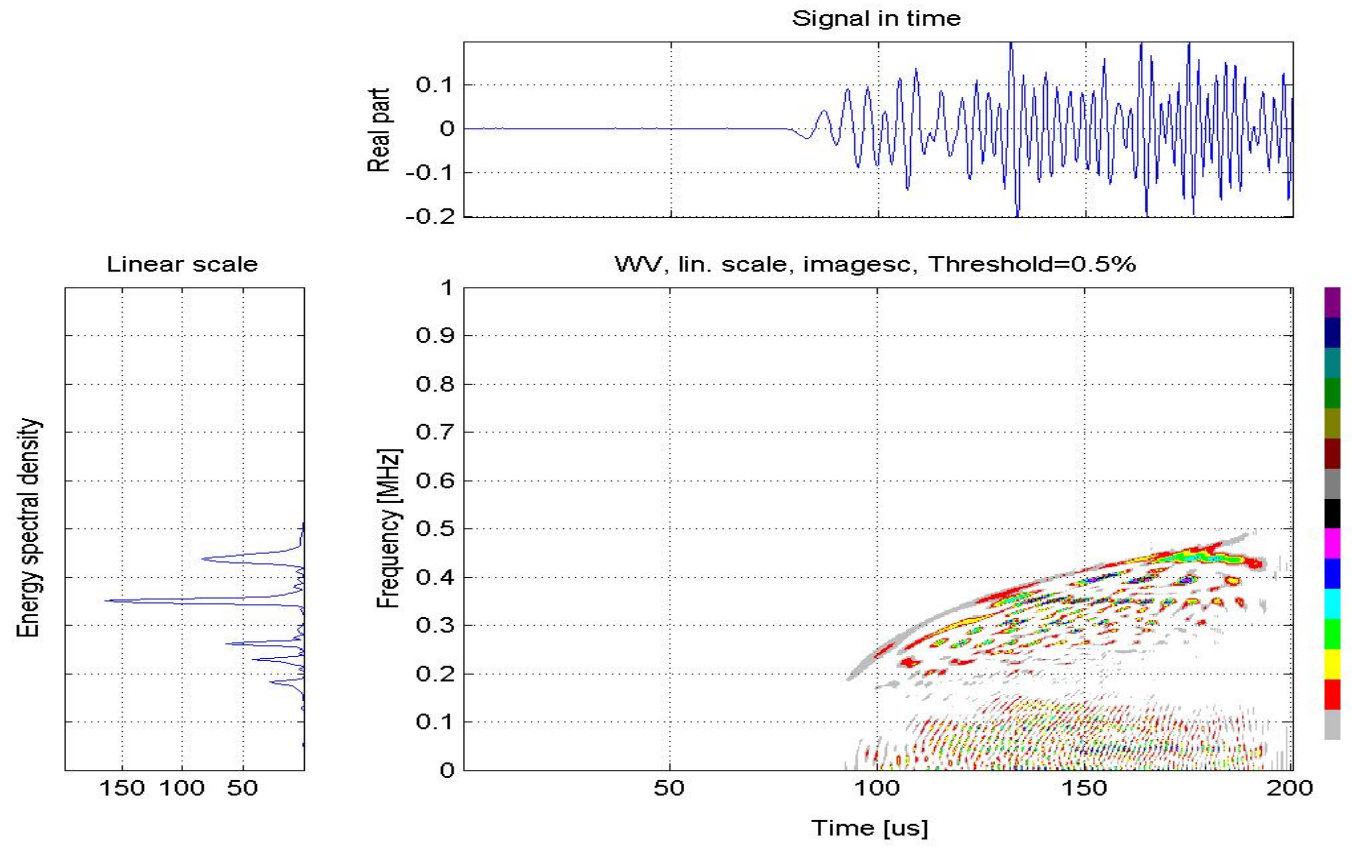

Figure 5.40 The Wigner-Ville Transform applied on experimental result due to the C101HV pulse generator input on the 3 meters rod
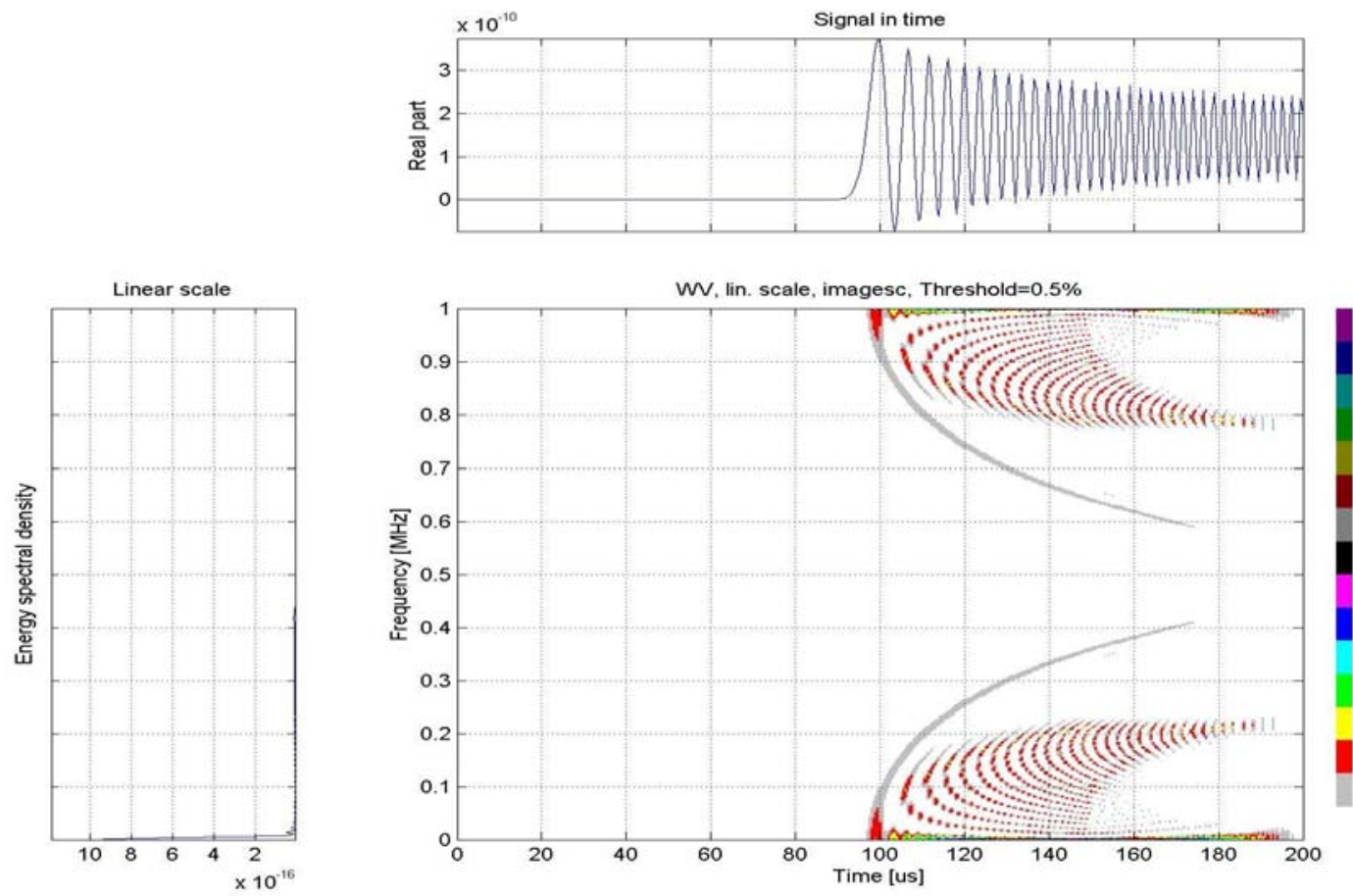

Figure 5.41 The Wigner-Ville Transform applied on FEM result due to the C101HV pulse generator input on the 3 meters rod 


\section{CHAPTER 6 \\ CONCLUSIONS AND RECOMMENDATIONS}

\subsection{CONCLUSIONS}

In this study, an effective FEM model to simulate wave propagation in an elastic solid rod was successfully generated. The accuracy of the FEM simulation is verified by different element size, the frequency of input waveform and the material properties. In order to measure the waveform in a solid rod, a stress wave is sent through it by utilizing a pulse generator. The experimental results and FEM output via waveform, wave velocity, frequency content, and attenuation are compared. All of the three rods are Grad 250 rods. The short rod $(158.75 \mathrm{~mm})$ and 3 meter rod specimens are the center wires of seven-wire strands; and the 4.76 meter long rod is a seven-wire stress-relived strand. Three different specimens and two kinds of input waveforms are presented. One was generated by the DS345 function generator. The waveform contains 10 sine waves that were windowed by one half of a sine wave. The frequencies of the sine waves are set to be $308 \mathrm{kHz}, 364 \mathrm{kHz}$, $400 \mathrm{kHz}, 444 \mathrm{kHz}$, and $500 \mathrm{kHz}$. Another input used was the square wave generated by the C101HV pulse generator. Experimental results indicated that the longer rod is better for observing the dispersion of the frequency components. However, longer rods presented some limitations such as energy attenuation and wave distortion. The short rod shows the arrival part of a waveform clearly, but it is not long enough to show the frequency separation.

Algor software is used as a FEM computer simulation program in this study. Dynamic linear-elastic analysis is conducted using step-by-step time integration method. The wave propagation in an elastic homogenous isotropic solid rod is modeled using 3-D 
8-Nodes or 2-D 4-Nodes Axisymmetric elements. A time step $(\Delta \mathrm{t})$ of $0.01 \mu \mathrm{sec}$ was applied and the element size in the longitudinal direction is $0.159 \mathrm{~mm}$ for the steel rod. For the aluminum bar, the time step $(\Delta \mathrm{t})$ of $0.02 \mu \mathrm{sec}$ was applied and the element size in the longitudinal direct is $0.2032 \mathrm{~mm}$. The aluminum bar experiment results compare well the FEM results and the theoretical results assure that FEM works well for wave propagation problems. The Axisymmetric model produces same result as 3-D model. Especially in the long rod simulations, the outputs can nicely show that the waves can be dispersed by different frequencies. Results show that the FEM model is able to consider the elastic waveguide theory and the acoustoelastic effect. Both experimental and FEM simulation result for the steel rods were compared in time-amplitude and time-frequency domain using WVT. The FEM numerical results correlated well with the experimental result. By using the WVT as the signal process tool, both frequency and time information could be shown at the same time. Therefore, the waveforms in time-frequency domain provide a clear comparison in frequency component and the arrival time of each frequency. In summary, the FEM simulation for wave propagation in solids provides us an efficient reference to real test.

\subsection{RECOMMENDATION FOR FUTURE STUDY}

In the future study, we can consider the whole wave propagation procedure as a transfer function (T(f)). This function is a frequency-domain function and related to the dimensions and material properties of the rod. The transfer function is defined by Eq. 6.1

$$
T(f)=\frac{O(f)}{I(f)}
$$


where $\mathrm{O}(\mathrm{f})$ and $\mathrm{I}(\mathrm{f})$ are the output and input in the frequency domain. $\mathrm{O}(\mathrm{f})$ and $\mathrm{I}(\mathrm{f})$ can be obtained by using Fourier Transform on $\mathrm{O}(\mathrm{t})$ and $\mathrm{I}(\mathrm{t})$ where $\mathrm{O}(\mathrm{t})$ and $\mathrm{I}(\mathrm{t})$ are output and input waveform. It is obvious that both experiments and FEM simulations has their own transfer function and we can mark them as $\mathrm{T}_{\mathrm{e}}(\mathrm{f})$ and $\mathrm{T}_{\mathrm{f}}(\mathrm{f})$. In addition, the relationship between experiment and FEM can be linked by calculating R(f) as given by Eq 6.2

$$
R(f)=\frac{T_{e}(f)}{T_{f}(f)}
$$

One of the purposes of using the DS345 function generator is that we can generate inputs with narrow band frequency. Since the ten sine waves windowed by one half sine is used, the frequency component of these sine input are no longer a single number. For example, after ten $400 \mathrm{kHz}$ sine waves windowing by a half sine wave, the frequency of these waves will be from $360 \mathrm{kHz}$ to $440 \mathrm{kHz}$. This means that the half sine windows could conduct $\mathrm{a} \pm 10 \%$ difference to frequency. In the future study, another window function, Hanning function, could be employed in order to make the input frequency as close to the original consideration as possible.

It is known that the strand's material constants, such as Poisson ratio and mass density, have evident effect on the ultrasonic wave propagation. Due to the uncertainty of material constants, the FEM simulation could not generate accurate output waveform. In future study, these material constants can be obtained by having a material test. Having real material constants, the FEM results could be more correspond with the experimental one.

The ultimate goal of this study is to simulate the wave propagation in the tendon which is under tensile force. After comparing the experiment and FEM result, we are able 
to estimate the residual force inside the tendon. In the future study, the boundary conditions can be added in order to make the model under stress.

In addition, due to the limitation of hard drive space, the successful simulation of ultrasonic wave propagation can only go up to about 3 meters by using the Algor software with 200 GB hard drive. Nevertheless, this length is not long enough compared with the common tensile tendon used in current fields. In the future, perhaps we can use other programs which are more efficient in dealing this kind of wave propagation problems. 


\section{REFERENCES}

1. Chen, H.L., Shah, S.P., and Keer, L.M., "Dynamic Response of Shallow-Buried Cylindrical Structures," Journal of Engineering Mechanics, Vol. 116, No. 1, pp. 152-171, January, 1990.

2. Chen, H.L., and Wissawapaisal, K., "Measurement of Tensile Forces in a Seven-Wire Prestressing Strand Using Stress Waves," Journal of Engineering Mechanics, Vol. 127, No. 6, pp. 599-606, 2001.

3. Chen, H.L., and Wissawapaisal, K., "Application of Wigner-Ville Transform to Evaluate Tensile Force in Seven-Wire Prestressing Strands," Journal of Engineering Mechanics, Vol. 128, No. 11, pp. 1-9, November, 2002.

4. Firestone, F.A., Flaw Detecting Device and Measuring Instrument, US Patent, 2,280, 226, 1942; and 2,280, 130, 1940.

5. Flanagan, D.P., and Belytschko, T., "A Uniform Strain Hexahedron and Quadrilateral with Orthogonal Hourglass Control," International Journal for Numerical Methods in Engineering, Vol. 17, pp. 679-706, 1981.

6. Folk, Robert, Shook, C.A., and Curtis, C.W., "Elastic Strain Produced by Sudden Application of Pressure to One End of a Cylindrical Bar. I. Theory," The Journal of the Acoustical Society of America, Vol. 30, No. 6, pp. 552-558, June, 1958

7. Fox, George, and Curtis, C.W., "Elastic Strain Produces by Sudden Application of Pressure to One End of a Cylindrical Bar. II. Experimental Observations," The Journal of the Acoustical society of America, Vol. 30, No. 6, pp. 559-563, June, 1958.

8. Goudreau, G.L., and Hallquist, J.O., "Recent Developments in Large-Scale Finite Element Latgangian Hydrocode Technology," Computer Method in Applied Mechanics and Engineering, Vol. 33, pp. 725-757, 1982.

9. Kolsky, H., "The Propagation of Longitudinal Elastic Waves Along Cylindrical Bars," Philosophical Magazine, seventh series, Vol. 45, no. 366, pp. 712-726. 
10. Hughes D.S., and Kelly J. L., "Second-Order Elastic Deformation of Solids," Physical Review, Vol. 92, No. 5, pp. 1145-1149, 1953.

11. Meitzler, H. Allen, "Mode Coupling Occurring in the Propagation of Elastic Pulses in Wires," The Journal of the Acoustical Society of America, Vol. 33, No. 4, pp. 435-445, April, 1961.

12. Miklowitz, J., and Nisewanger, C. R., "The Propagation of Compressional Waves in a Dispersive Elastic Rod,” Journal of Applied Mechanics,” Vol. 24, pp. 240-244, 1957.

13. Oz, Jasmin, and Heyman, Ehud, "Wigner-Ville distribution for pulse propagation in random media: pulsed plane wave," Waves in Random Media, Vol. 8, No. 2, pp. 175-191, 1998.

14. Sansalone, Mary, and Carino, J. Nicholas, "A Finite Element Study of Transient Wave Propagation in Plates," Journal of Research of the National Bureau of Standards, Vol. 92, No. 4, pp. 267-278, July-August, 1987.

15. Sokolv, S., and C. Rosenberg, "NETtalk: a Parallel Network that Learns to Read Aloud," Johns Hopkins University Tech. Rep. JHU/EECS086/01, 1986.

16. Wissawapaisal, Komwut, "Nondestructive Testing of Reinforced and Prestressed Concrete Structures Using Acoustic Waveguides," Dissertation, WVU, Morgantown, WV, 2001. 


\section{Appendix A: Experiment Setup}

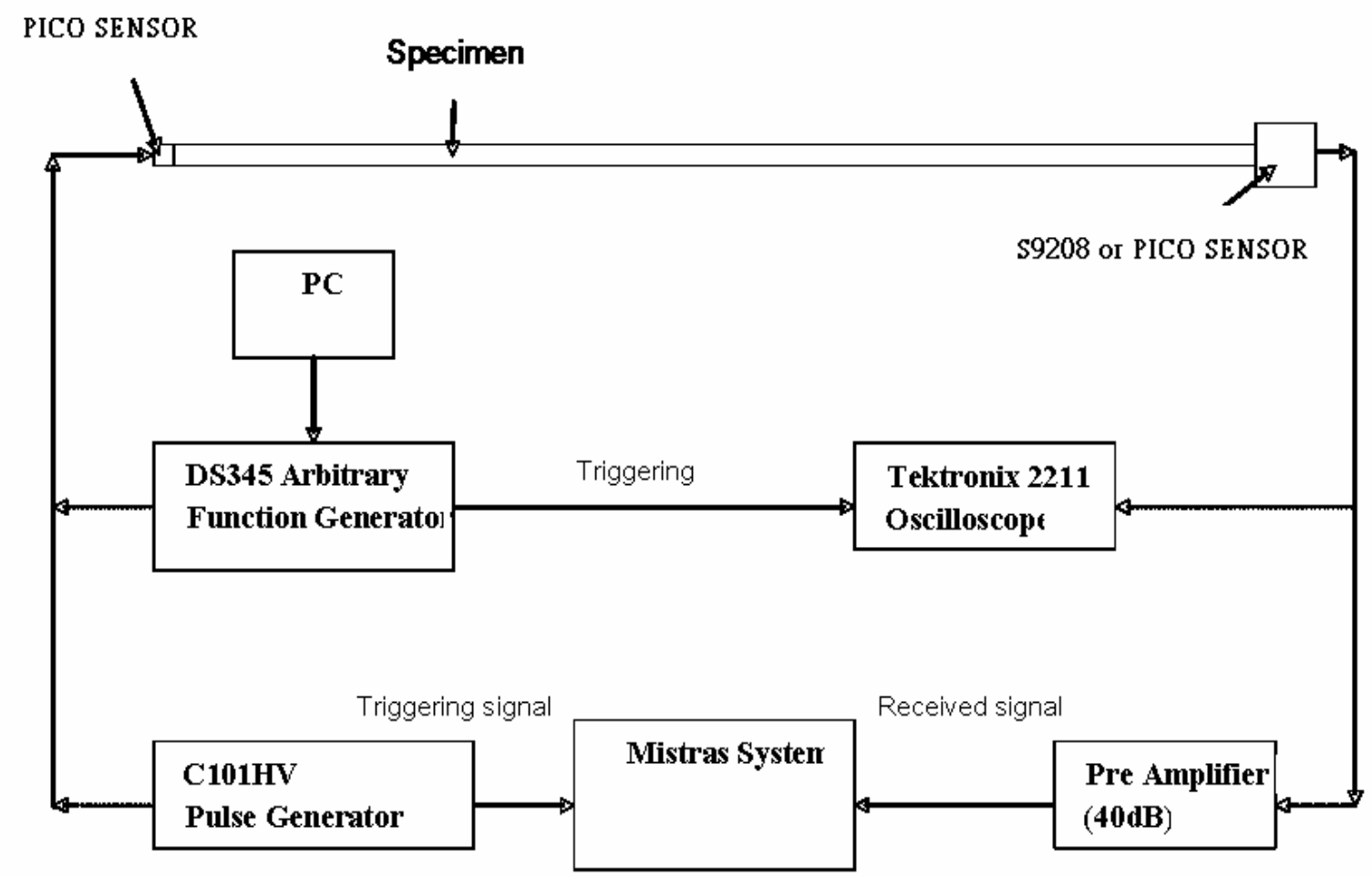

